ruticry, su o 715 Wharton Struet, Philada, Pa.

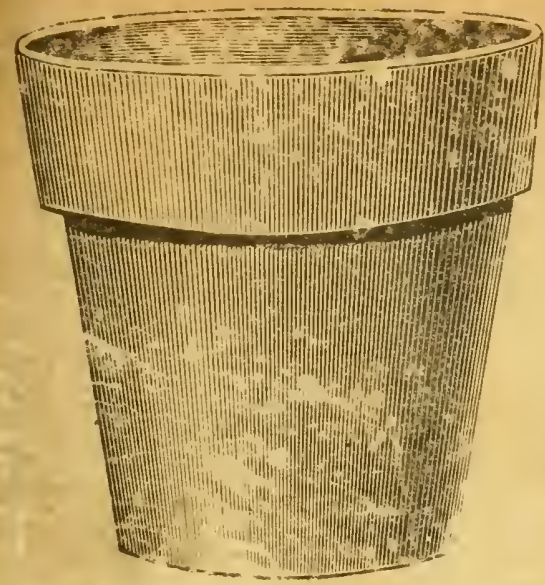

Pel:ering that Flower Pots could be made oy si: " which w wuid ive greater strength and at t':e rame in, greater porosity, we commenced in a stacll: $\mathrm{y}, \mathrm{y}$, in 3377 , to make them, and have contus.er aking fro $h_{4}$ time to time, such im. prowaci.ts as irpi ance and observatiousugQcoted, un:il in save reached a; grear a degree ui perfoctio i ir cis: mode of manufacture, in porosity and in cur-bility, as is possihle. The demand for Flower Pots increased so rapidy that we were compeiled to enlarge our building and add macninery equivalent to five times our former capacity. We nov take pleasure in announcing that we are prepared to fill all orders with fromptness and disparch. A full line of Flover Pots and ail ware regularly in stock.

Sond for lilustrated Price List.

\title{
Horticultural Directory
} of the

\section{United States.}

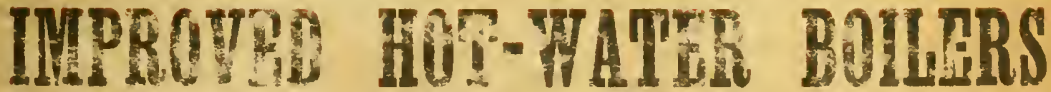

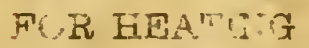

GREENHOUSES, GRAPERIES, EtC.

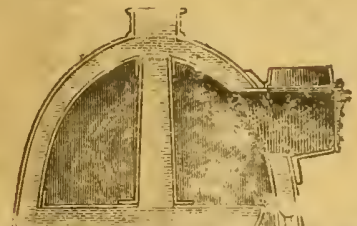

11.

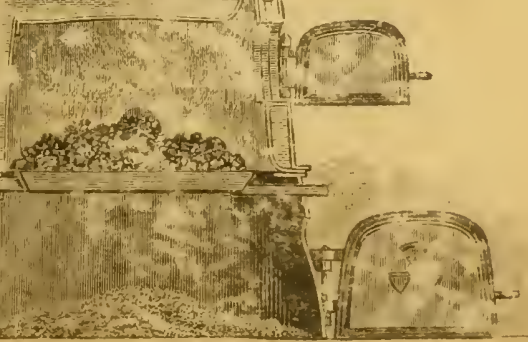

Cast Iron Pipe and

Pipe Fittirģs.

Galvanized ScrewEyes and Wire for Trellis Work.

Send For Cotalnrue.

Thos. W. Weathern:

46 Marion Strect.

NEW YCE:- 


\section{Ed.}

$124 \mathrm{~W}$.

NEV

MANUF.

Wire Desi

Baskets,

Etc.

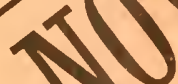

O]

$\mathbf{F}$

WIRE

BEST SHAPE.

SEND FOR PRICE LIST. LIBRARY

\section{$5 B$}

404

E83

\section{DATE DUE}

UNIV. OF MASSACHUSETTS/AMHERST 


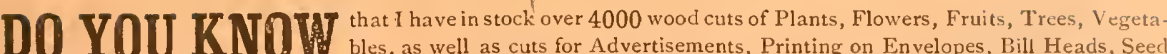

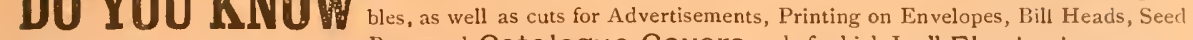

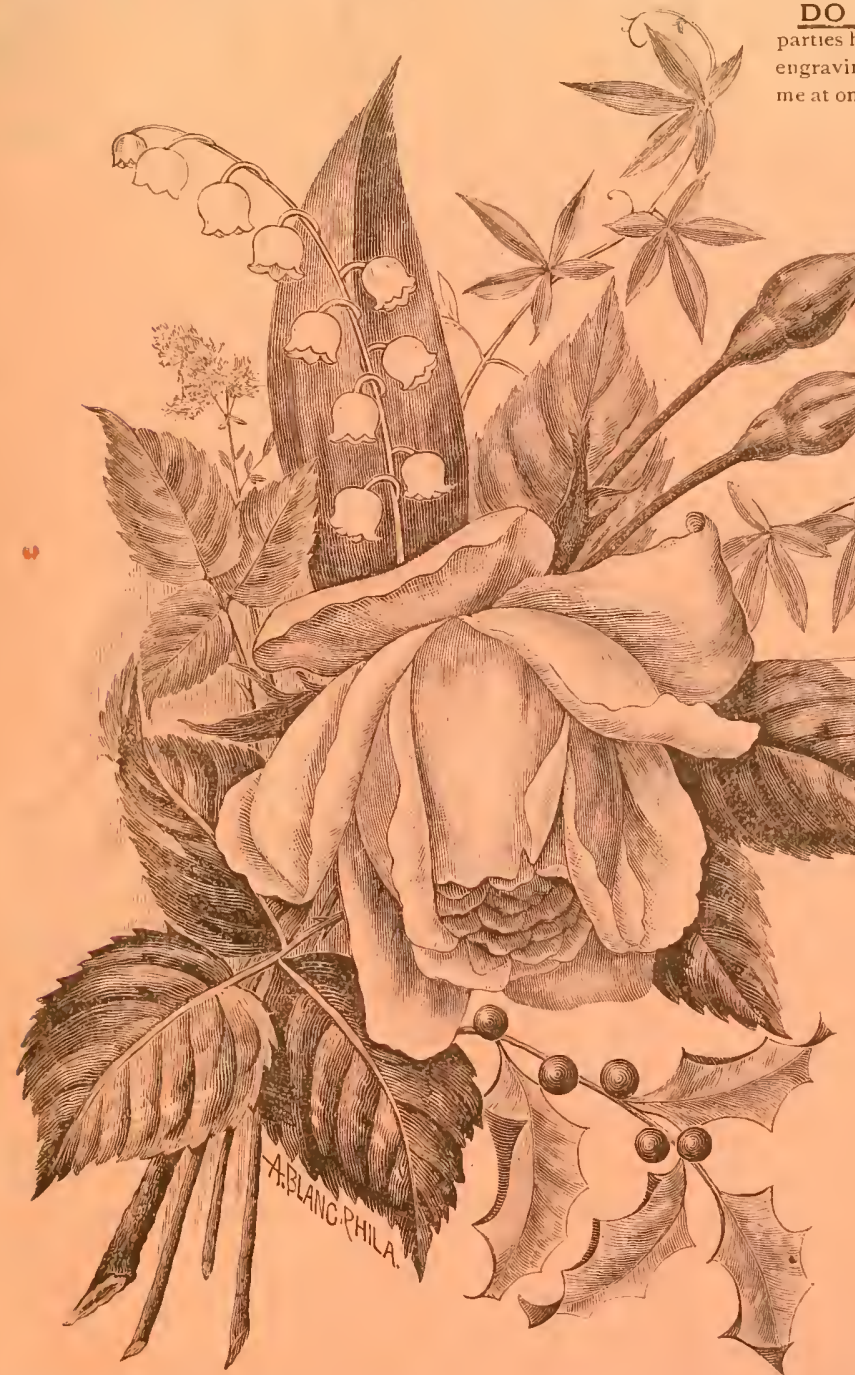
DO YOU KNOW that many pares have paid hundreds of dollars for gravings that they could have had of at one-tenth the price. Therefore, if al European Houses, avail themselves of my facilities and knowledge of the business and find it to their advantage to deal with me. For instance, suppose you have a New and Desirable Plant, Fruit, or Vegetable to introduce to the trade, and want an engraving of it, any other engraver may ask you from $\$$ ro to $\$ 50$ for the job, but, if (like the Parry Strawberry or the Bennett Rose for instance), I see that there is likely to be a demand for it, why, lipwould engrave you such a cut for Nothing. Can You Ask More.

Cuts and Lithographs for Catalogue Covers in stock and inade to order. Book of specimens 15 cents. Views of Nurseries in the best style at low prices, for specimens see Messrs. H. A Dreer, Storrs \& Harrison, W. \& T. Smith, C. Young \& Sons, and others. Colored plates in quantities of five hundred and upwards at from $\$ 350$ per thousand. In fact, anything in the Electro, and Engraving Line at Lowest Prices.

\section{A. BLANC, Philadelphia,}

Engraver and Electrotyper for Florists, Seedsmen, and Nurserymen. 

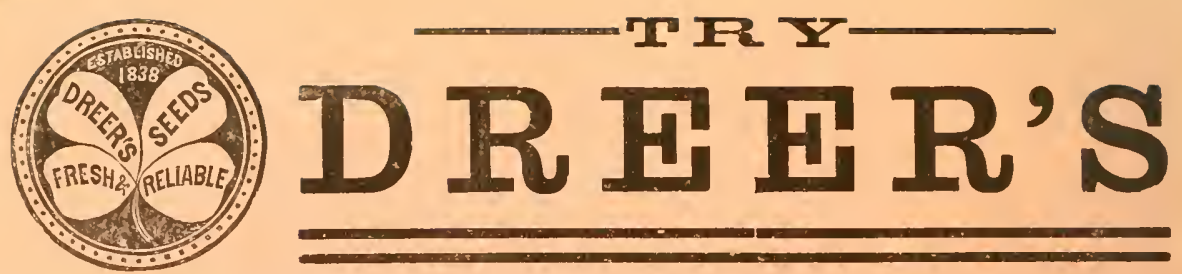

VEGETABLE SEEDS,

FLOWER SEEDS. Specialties of our own saving, or grown under contract by well-known responsible FLOWER SEEDS. raisers.

PI Varieties of home or foreign origin are offered as soon as they appear. Our correspondents in all PLANTS. parts of the world place us in this position.

BUL raised on own Nursery. or imported direct from growers of established reputation in Holland, France, BULBS, Italy, Cape of Good Hope, and Japan.

GARDEN REQUISITES, of well tried and modern introductions, for labor saving, profit, and pleasure.

TRIAL AND NURSERY GROUNDS are well located and organized to test and experiment in old and new varieties in every department. Here the experience is acquired to protect our customers from imposition and loss from spurious, defective, and worthless varieties.

DREER'S GARDEN CALENDAR, published January of every year, giving complete lists of everyDREER'S BULB CATALOGUE, issued in September, offering Dutch Bulbs and Winter-blooming WHOLESALE LIST TO THE SEED AND PLANT TRADE, issued quarterly.

All the above Catalogues mailed free on application.

HENRY A. DREER, Seedsman and Florist, 714 Chestnut St., Philada. Grein-House Heating and Ventilating. HITCHINGS \& CO.,

(ESTABLISHED 1844.)

No. 233 Mercer Street, New York.

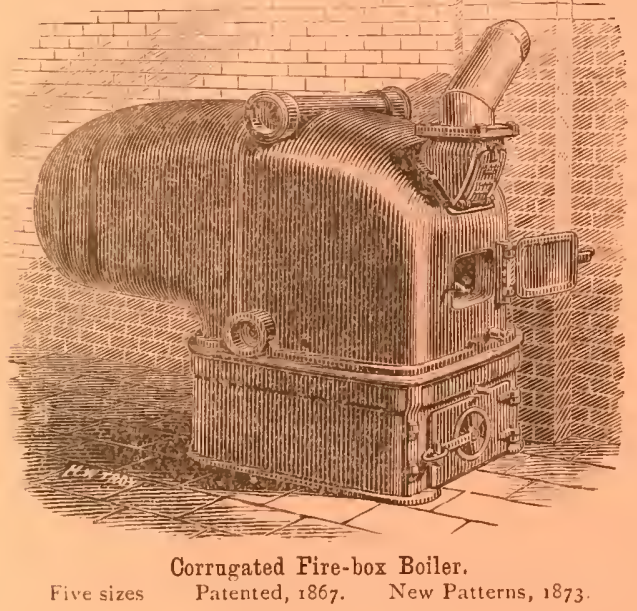

FOUR PATTERNS OF BOILERS, Eighteen Sizes.

SELF-FEEDING HEATERS, For Small Conservatories.

HEATING PIPES and FITTINGS.

SASH-RAISING APPARATUS AND LIFTING RODS.

Send 4 cents postage for Illustrated Catalogue and List of References. 


\section{HORTICULTURAL DIRECTORY}

OF THE

\section{UNITED STATES.}

VOL. I.-(FLORISTS AND NURSERYMEN.)

ALPHABETICALLY ARRANGED BY NAMES AND STATES. (INTERLEAVED.)

PRICE, SIX DOLLARS.

EDITOR :

CHARLES F. EVANS, ROWLANDVILLE NURSERIES, PHILADELPIIA.

Entered according to Act of Congress in the year 1885 by Isinc D. SAlLer, in the trfice of the Librarian of Congress at Washington, D. C.

PHIJ, A D ELPHIA:

PUBLISHED BY ISAAC D. SAILER. 1885. 


\section{HEADQUARTERS}

We are generally admitted to be in the foremost ranks in producing and distributing the following Plants and Bulbs:

Geraniums, Chrysanthemums, Carnations, Fuchsias, and all classes of New Plants.

Gladioli, Lilies, Tuberoses, Dahlias, Tigridias, and numerous other Bulbous Rooted Plants.

\section{Various Plants of Merit are always to be had.}

Specialties in Choice Seeds, as

Balsams, Zinnias, Primulas, Calceolarioes, etc. suitable for Florists' Use.

\section{DUTCH BULBS}

Of the very best quality only.

Our Hyacinths, Tulips, Narcissus, and Lily of the Valley are selected with only one object, that is to secure the best only.

1. We do not claim to sell goods below cost or without C. U. profit, but alway's endeavor to give value for prices asked. We issue Four Trade Catalogues each year, and from those not already dealing with us we solicit a share of their patronage. Respectfully,

\section{H. HALLOCK, SON \& THORPE, QUEEISS, IV. T.}

Letters should be addressed to East Hinsdale, N. Y.; and telegrams to QUEENS, N. Y. 


\section{PUBLISHER'S PREFACE.}

ONLY one who has undertaken the task can fully appreciate the difficulties to be overcome, the tedious details to go through, in order to compile a "Trade Directory." The new people going into the business, the old people going out, the changes in firm names and locations, new street numberings, etc., all stand in the way to prevent the publisher producing a perfect book. In offering this "Directory" to the trade, the Publisher feels that he is supplying a long-felt want. While aiming at perfection, he believes that he has fallen short, as all must do in such undertakings. Expense, labor, patience, and a long apprenticeship to this kind of work, have all been freely expended. IVith all these aids, he realized at the start that there would be many errors of omission and commission, and he therefore early determined to issue only interleaved copies of the "Directory," and to supplement the same with correction sheets, to be issued from time to time-annually only, it is expected. By this means he hopes finally to attain perfection, and then maintain it. He respectfully and earnestly asks of the trade to assist in this laudable ambition by using the enclosed envelope to inform him of such errors as may be known to them.

The trade must bear in mind that this "Directory" only purports to give the names and addresses of the Nurserymen and Florists. Seedsmen, Vineyards, Tree Dealers, etc., we do not pretend to include. Many, no doubt, are in the book; either it is a mistake, or else they combine something of the Nurseryman or Florist with their other business.

I cannot close these remarks without returning thanks, and acknowledging my obligations, to the several very prominent members of the trade who have kindly assisted in the final revision of this work.

Very respectfully,

ISAAC D. SAILER, Publisher.

Union Insurance Co Building, $3 \mathrm{~d}$ and Walnut Sts Philadelphia, PA., March, 1885.

All communications should be addressed to the Publisher. Correspondence solicited.

NoTE.-In the list of names, $n$ is for Nurseryman, and $f$ for Florist. 


\section{WELCH BROTHERS,}

\section{Wholesale ${ }^{a n d}$ Commission Florists,}

No. 165 Tremont Street, Boston, Mass.

All varieties of Fancy Roses and other Fresh Flowers carefully packed and selected for shipment to points in Western and Middle States at the lowest wholesale prices. All orders by mail, express, telegraph, or telephone will receive prompt attention.

Price List of Cut Flowers Furnished on Application.

\section{FLOWERS RECEIVED FOR SALE ON COMMISSION.}

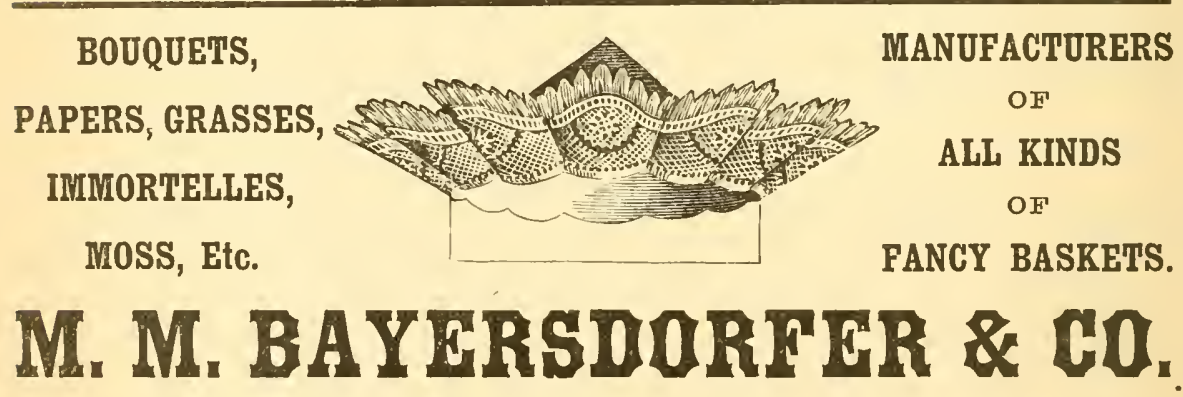

Importers of Florists' Supplies,

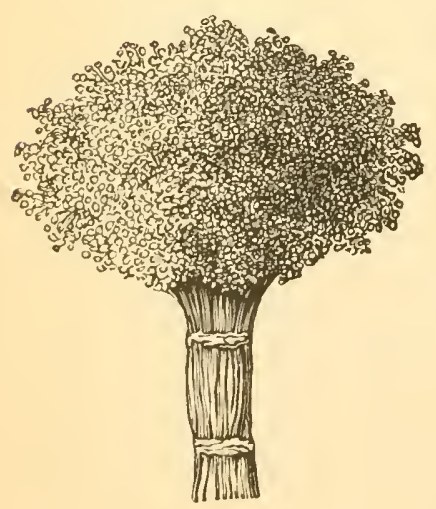

56 N. Fourth Street,

PHILADELPHIA.

CATALOGUES

EURNISHED

ON

APPLICATION.

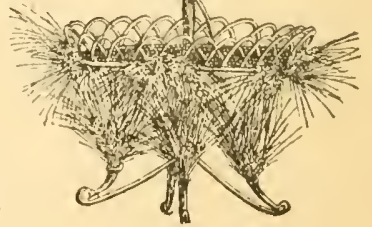




\section{CONTENTS.}

Title Page

Preface

Contents .

Index to Advertisers

Alabama .

Alaska Territory

Arizona Territory

Arkansas

California

Colorado

Connecticut

Dakota Territory

Delaware

District of Columbia

Florida

Georgia

Idaho Territory

Illinois

Indian Territory

Indiana

Iowa

Kansas

Kentucky

Louisiana

Maine

Maryland

Massachusetts

Michigan

Minnesota

Mississippi

Missouri

Montana Territory .

Nebraska

Nevada

New Hampshire

New Jersey

New Mexico Territory

New York

North Carolina

Ohio

Oregon

Pennsylvania

Rhode Island .

South Carolina

Tennessee

Texas

Utah Territory

Vermont .

Virginia

Washington Territory

West Virginia .

Wisconsin

Wyoming Territory 


\section{W. S. ALLEN,}

\section{Wholesale Florist,}

940 Broadway, New York.

๑ PRICE LISTS SENT TO ALL APPLICANTS. -

\section{FINE ROSES A SPECIALTY}

\section{HOOKER NURSERIES,}

ESTABLISHED 1835 .

H. E. HOOKER COMPANY, PROPRIETORS,

\section{ROCHESTER, N. Y.}

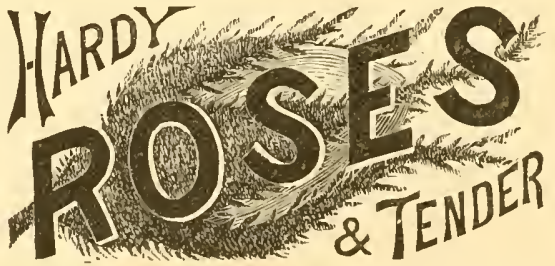

A Full Line of Fruit and Ornamental Trees, Vines, Shrubs, etc. etc.

GRAPE VINES AND ROSE BUSHES

A SPECIALTY. 


\section{INDEX TO ADVERTISERS.}

Allen, W. S.

Bayersdorfer, M. M.

Blanc, A.

Bowker Fertilizer Co.

Burpee, W. A., \& Co.

Cassell, J. C.

Clark \& Bros.

Clark \& Nettleton

Corcoran, A. J.

Craig \& Bro.

Cranston's Nursery and Seed Co. (England)

De Veer, J. A.

Dreer, Henry A.

Ellis, Charles R.

Ellwanger \& Barry

Evans, Charles F.

Exeter Machine Co.

Fennell, J. P.

Fergusson, D., \& Son

Gardeners' Monthly

Green, L., \& Son

Hallock, V. H., Son \& Thorpe

Hammond \& Hunter

H. E. Hooker Co.

Hews, A. H., \& Co.

Hitchings \& Co.

Horticultural Advertiser (England)

Jansen, Edward

Jones, A. L.

King, James

Ladies' Floral Cabinet

Marschuetz \& Bacharach

Moon, Samuel C.

Nanz \& Neuner

Parker \& Wood

Parsons \& Sons Co.

Pennock, Charles E.

Perkins, John J.

Pierson, F. R.

Reed \& Keller

Sailer, Isaac D.

Scollay, John A.

Siebrecht \& Wadley

Smith, Richard \& Co. (England)

Smith, William $\mathrm{H}$.

The American Garden

The "Bennett" Rose

Union Insurance Co.

Van der Veur, P. W.

Vaughan, J. C.

Weathered, Thomas W.

Welch Bros.

Whilldin \& Co.

Back cover

IO3

IO2

Back cover

IOO

104

8

I08

v

99

IOI

9

Front cover

Back cover

v

8

IOI

8

I06

ii

100

IOI

IOI

IOI

108

IOI

Facing page 8

Front cover

Front 4

Front cover 
JAMES KING, 59 \& 61 Lake Street, Chicago.

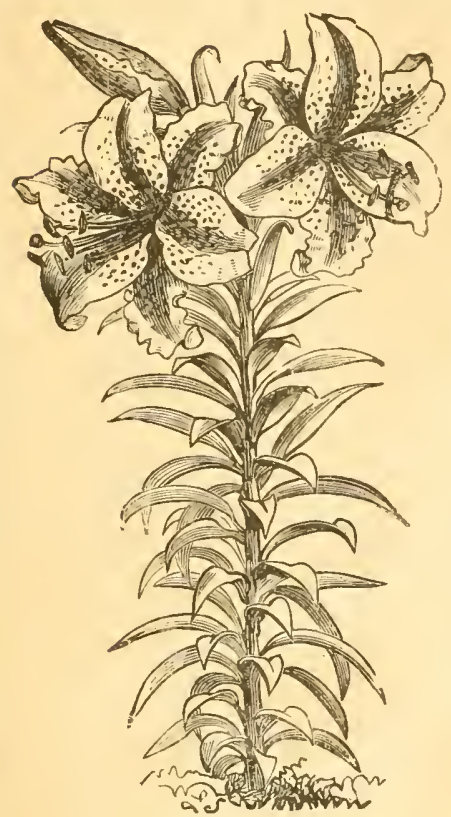

Lilium suratum.
SPRING and PALL BULBS

\author{
LILIES, \\ TUBEROSE, GLADIOLUS, \\ HYACINTHS,
}

TULIPS, NARCISSUS, Etc.

Fine Florists' Seeds,

Seeds for Market Gardenels: Plopists' slulluies,

Immortelles, Grasses, Mosses, Wire Work. Rustic Work,

Bouquet Papers.

Baskets in Large Variety.

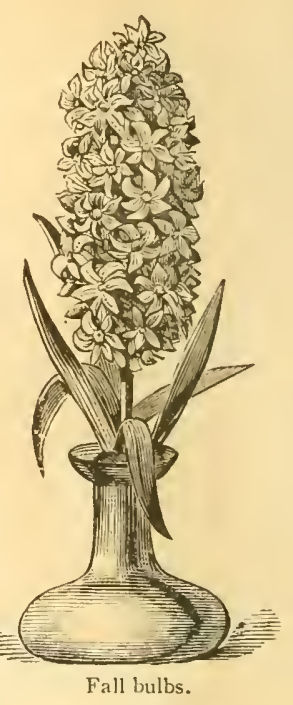

Send for Wholesale Catalogues.

\section{TO IMPORTERS OF ROSES.}

Cranston's Nursery and Seed Co., Limited, PROPRIETORS OF

\section{THE LARGEST ROSE GROUNDS IN ENGLAND,}

Have over HALF A MILLION ROSE PLANTS forsale, and are prepared to book orders for delivery in the Fall. All the leading varieties SUITABLE FOR FORCING, can be supplied in large as well as small quantities, as we grow from $\mathbf{1 0 0 0}$ to $\mathbf{1 0 , 0 0 0}$ of each sort. Visitors to England are cordially invited to inspect our Nurseries, and we shall be pleased to answer any inquiries as to the best and nearest route, etc. Before giving away your orders, write for our Catalogues, which will be mailed free of charge. Address,

CRANSTON'S NURSERY AND SEED CO., Limited,

KING'S ACRE, HEREFORD, ENGLAND.

\section{ADVERTISEMENT.}

\section{HORTICULTURAL ADVERTISER.}

We beg to call the attention of all American Nurserymen and Seedsmen to the above paper, the only advertising medium to the Horticultural Trade. The Horticultural Advertiser is published weekly, and sent gratis to every Nurseryman and Seedsman in Great Britain, and six hundred of the principal Continental Houses. Annual fee for postage, two shillings and sixpence. All American houses doing an export or import trade, will find this paper of the utmost value to their business. Specimen copy free on application. 


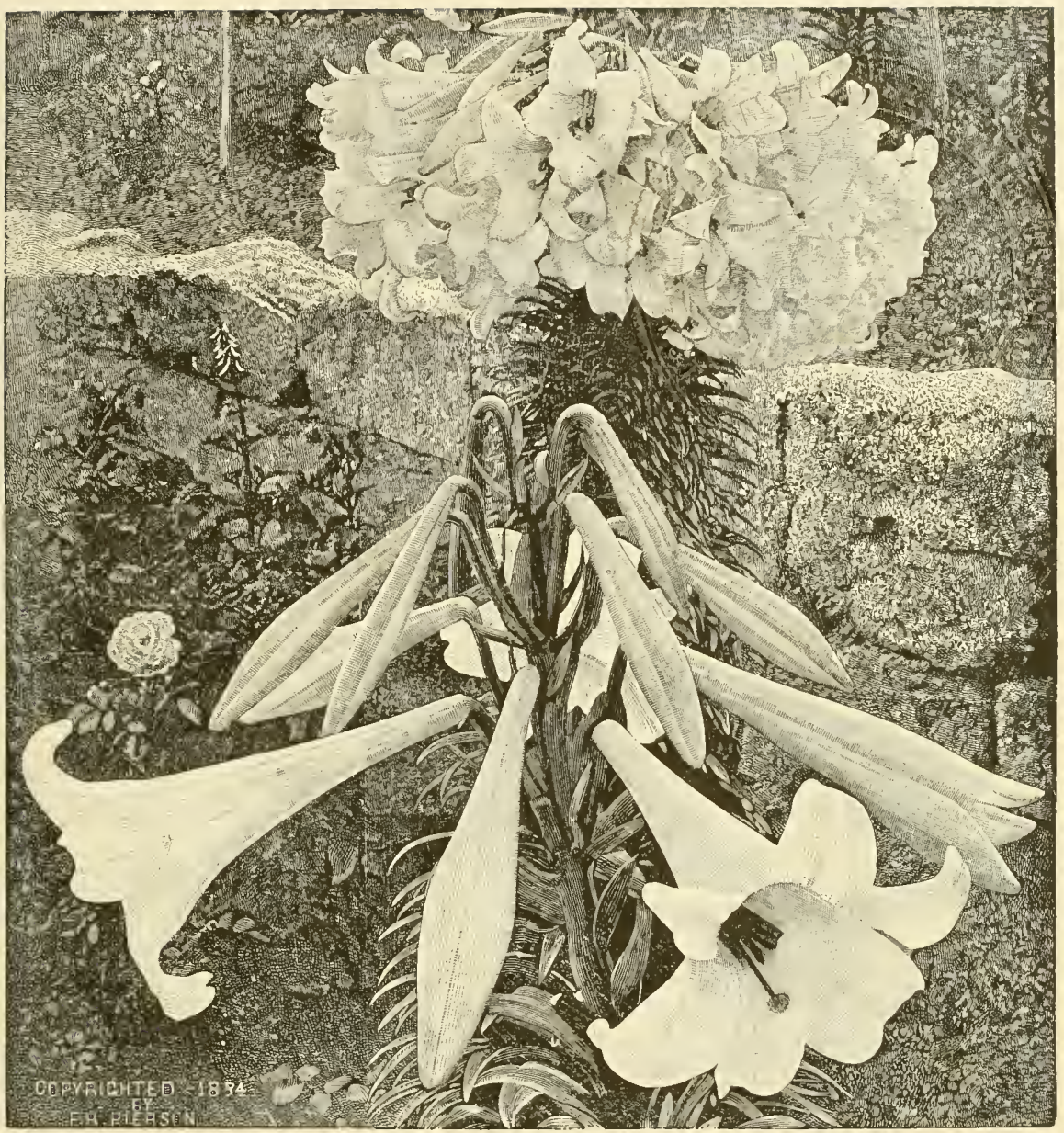

FROM A PHOTOGRAPH TAKEN IN BERMUDA, SHOWING EXTRAORDINARY DEVELOPMENT OF A PLANT, WITH 145 BLOSSOMS ON A SINGLE STEM; AN AVERAGE PLANT IN THE FOREGROUND.

\section{LI U M HARRISSI,}

BETTER KNOWN AS

\section{THE BER M U DA EASTER LILY.}

The Best in the World for Forcing for Winter Flowers.

We Offer only Strong Field-Grown Bulbs from Our Own Grounds in Bermuda.

This is not only the best by far of all Lilies for Winter blooming, but it is one of the most profitable flowers that can be grown by florists Notwithstanding the extreme depression in the cut-flower market the past winter, the demand for this flower warranted dealers in paying us as high as $\$ 6.00$ per dozen blooms, and notwithstanding the immense number of plants we flowered, we found at all times a ready demand at large prices for our entire product. It is very easily handled, and the flowers being especially effective for decorative purposes, always command large prices. It derives its name from the fact that, in Bermuda, grown in the open ground, it blooms at Easter time, but, in the greenhouse with successive lots, it can be had in bloom all the Winter from early in December until after Easter; in fact, by special, culture atl the year round. THE FACT THAT IT CAN BE FORCED INTO BLOOM BY THE CHRISTMAS HOLIDAYS adds particularly to its value, as it fills in at a time when flowers are usually scarce and in great demand.

For this purpose our Bermuda groun bulbs are indispensable, as in Bermuda the bulb reaches its highest development and ripens off perfectly, and is ready for shipment by the middle of July, before bulbs in our own country have hardly begun to make their growth. Our bulbs being grown in the open ground, without artificial culture or manure of any kind, are particularly strong and healthy, and calculated to give far better results than those grown by other growers in greenhouses in pots.

We are Headquarters for this Lily and Supply the Trade in this Country and in Europe.

We were the first to offer it at a reasonable price and to grow it in very large quantities, and we hold not only by far the largest, but, in fact, nearly the entire stock of this valuable Lily. New crop will be ready for delivery August rst. Orders booked now for delivery at that time. Wholesale price on application. In order to gain the full value of this Lily it should be potted early and so secure it in flower by the Holidays.

\section{F. R. PIERSON \& CO.,}





\section{The "Bennett" Rose.}

\section{The New Crimson Tea.}

ThE undersigned, the Importer and Sole Owner of this world-famous rose, offers plants to the trade at market prices.

For quotations, apply to

\section{Charles F. Evans,}

Station F, Philadelphia, $\mathrm{Pa}$.

Prices will not be lowered before January I, I886, if then.

We reserve the right to advance rates without notice.

Correspondence solicited. 


\section{THE HORTICULTURAL DIRECTORY OF THE UNITED STATES.}

\section{ALABAMA.}

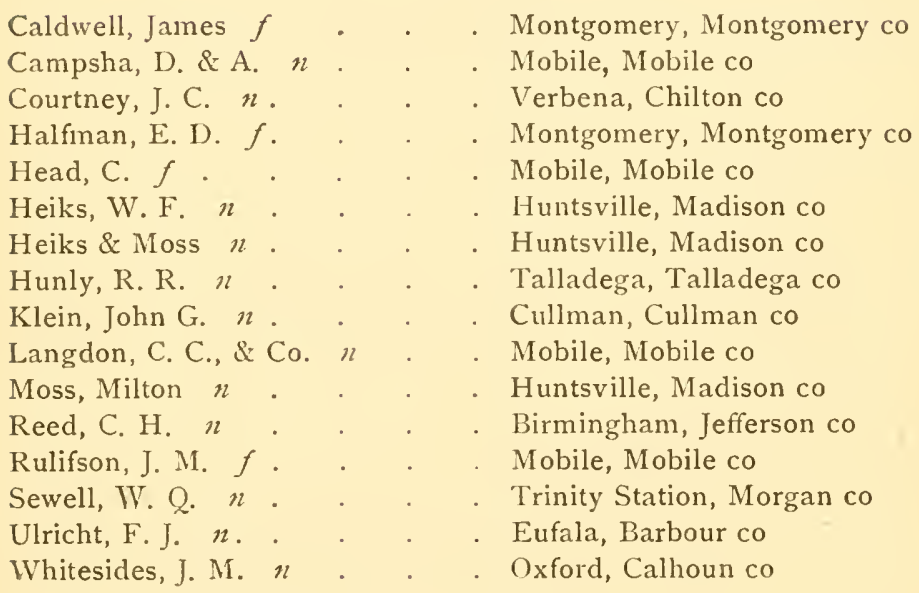

\section{ALASKA.}

\section{ARIZONA.}

Mason, A. $n$. . . . . Florence, I'inal co

Searle, H. S. $f$. . . Oro Blanco, Pima co 


\title{
ARKANSAS.
}

Archias, Leon $n$.

Babcock, E. F. $n$.

Birmingham, M. C. $n$

Bond, Joshua $n$.

Cates, F. P. n

Davis, B. F \& G C. n

Gill, John B., \& Co. $n$.

Gregory, C. H. $n$.

Hobbs, B. F, $n$.

Horn, D. G. H. $n$

Kirkman, WV. T. n

Legette, W.

Little, A. H. $n$.

Maggard, H. F. $n$

Matthews, S. J. $n$.

Morrow \& Son $n$.

Russell, J. M., \& Co.

Simons, Dr. J. F. $f$

Stromberg, William

Vestal, Joseph W. $f$

Welbourne \& Ayers $n$.

Whitman, James $n$

Woods, J. Walter $n$

Woolsey, Henry $n$

\author{
Fayetteville, Washington co \\ Little Rock, Pulaski co \\ Fort Smith, Sebastian co \\ Calico Rock, Izard co \\ Little Rock, Pulaski co \\ Bentonville, Benton co \\ Bentonville, Benton co \\ Altus, Franklin co \\ Clarksville, Johnson co \\ Liberty Springs, Van Buren co \\ Little Rock, Pulaski co \\ Batesville, Independence co \\ Nashville, Howard co \\ Elmwood, Boone co \\ Monticello, Drew co \\ Desare, Prairie co \\ Boonsborough, Washington co \\ Fayetteville, Washington co \\ Fort Smith, Sebastian co \\ Little Rock, Pulaski co \\ Paris, Logan co . \\ Dardanelle, Yell co \\ Malvern Junction, Hot Spring co \\ Bentonville, Benton co
}

\section{CALIFORNIA.}

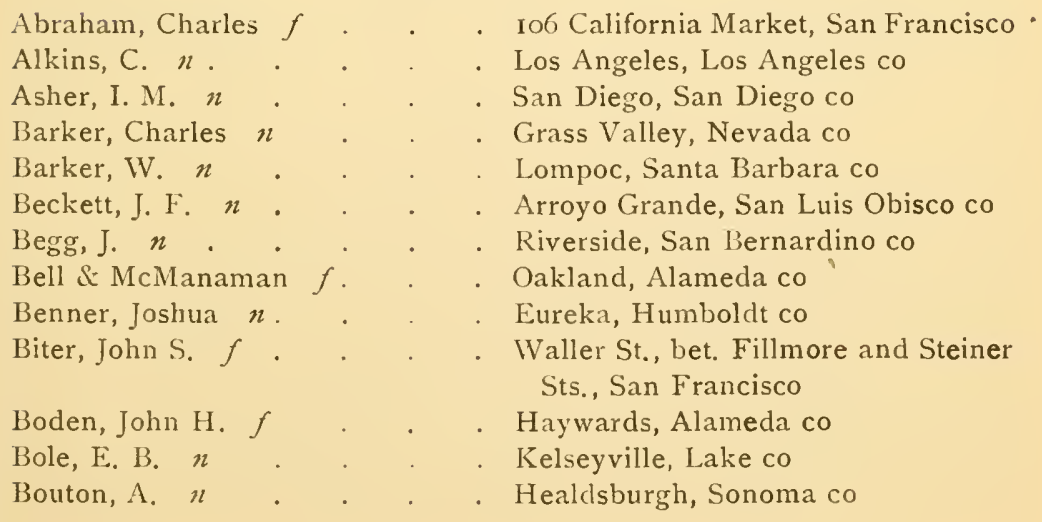


Bowen, E. J. $n$

Bowman Bros. $n$.

Buckner, Z. E. $f$.

Canning Co., J. Lush $n$

Card, J. E. $n$

Chapin, O. S. $n$.

Childs, O. W. $n$.

Collir, W. H. $f$

Cox, Thomas A., \& Co. $n$.

Culligan, John $f$.

Damon, W. C. $n$.

Daniels, Benjamin $f$

Dayton, C. N. $f$.

Dearborn, C. W. n

Deasy, Mrs. Jerome $f$.

Debicke, A. $f$.

Delbey \& Co. $f$.

Douglass \& Thorne $n$.

Doyle, Luke D. $f$

Duhem, August $f$

Eames, A. W. $n$.

Fahrenholz, Henry

Fautsch, F. E. $f$.

Ford, G, W. $n$.

Forsyth, John $f$.

Fowler, W. P. $n$.

Fox, R. D. $n$.

Franklin, M. $n$.

Fustini, Peter $f$.

Gaffenson, Elizabeth $n$

Gallo, B., \& Co. $f$.

Gallo, L., \& Co. $f$.

Garey, Thomas A. $f$

Gaubert, Madam Mary $f$

Geyer, Peter S. $f$.

Greget, L. $f$.

Harper, John $f$

Havens, H. B. $f$.

Herns, Mrs. Elizabeth $f$

Hibbert, Albert $f$.

Hogue, L. B. $n$

Howard \& Praiser $n$

Huchard, August $f$

Hutchinson, James $n$

Ingham, W. R., \& Son $n$
815 Sansome St., San Francisco

Gilroy, Santa Clara co

Santa Rosa, Shasta co

Oakland, Alameda co

Woodland, Yolo co

San Diego, San Diego co

Los Angeles, Los Angeles co

20 Post St., San Francisco

409 Sansome St., San Francisco

704 Turk St., San Francisco

Napa City, Napa co

409 Hayes St., San Francisco

P. O. Box 996, Los Angeles

Oakland, Alameda co

736 Market St., San Francisco

Alameda, Alameda co

252 Market St., San Francisco

Grangeville, Tulare co

N. E. cor. Central Ave. and Pine

St., San Francisco

I2 I Sutter St., San Francisco

Los Angeles, Los Angeles co

509 California St., San Francisco

Cor. Washington and Buchanan Sts.,

San Francisco

Santa Ana, Los Angeles co

20 Post St., San Francisco

Merced, Merced co

P. O. Box 847 San Jose, Santa Clara co

Carpenteria, Santa Barbara co

9 Eddy St., San Francisco

Sacramento, Sacramento co

246 Sutter St., San Francisco

69 Fourth St. and 109 Sixth St., San

Francisco

Los Angeles, Los Angeles co

6I 2 California St., San Francisco

Woolsey near Cambridge St., San

Francisco

Franklin and Greenwich Sts., San Francisco

San Francisco, San Francisco co 26 Nevada Block, San Francisco I03 Stockton St., San Francisco

15 Geary St., San Francisco

Carpenteria, Santa Barbara co 429 Montgomery St., San Francisco I 5 I4 Bush St., San Francisco Oakland, Alameda co

San Bernardino, San Bernardino co 
Jeannot, Francois $f$ Jones, George B. Kahn, Sarah $f$ King, M. $n$ Kohler, Bernard $n$ Kramer, Louis $f$.

Lanthéaume, John P. $f$ Leopold Bros. $f$. Lewis, Henry $n$ Livermore, H. P. Louideck, Mrs. M. Meherin, Thomas $n$ Meyer, Emile $f$ Meyer, William, \& Co. $f$. Miller, F. A. $n$ Miller, Sievers \& Co. Navlet, Charles S. Newman, Andrew $n$ Newsom, L. M. n O'Connor, Martin

O'Neil, J. $f$. Otoboni, B. $f$

Parish \& Bro. n Park \& Cassiday $n$ Parker Bros. Pasquier, L. $f$ Pepper, W. H. $n$. Pottet, H. $f$.

\section{Pouyal, Jean $f$}

Pouyallet \& Humbrecht $f$ Price, T. S. $f$ Pryal, A. D. $n$ Ranney, Rudolph $n$ Reed, C. W., \& Co. $n$. Robertson, William $f$. Rocks, John Sanders, W. A. n Sauchez, Felix $f$. Servel, Firman \& Co. Sevin, Vincent \& Co. $f$ Sexton, Joseph $n$. Short, W. N. n . Sievers, J. H. $f$. Silva, C. M., \& Son $n$. Silvester, George F. $n$. Sontag \& Co, n
I21 5 Geary St., San Francisco 20 Post St., San Francisco 716 Washington St., San Francisco Oakland, Alameda co St. Helena, Napa co

S. E. cor. Howard and Twenty-fourth Sts., San Francisco 2321 Mission St., San Francisco 35 Post St., San Francisco Carpenteria, Santa Barbara co Oakland, Alameda co 1229 Stockton St, San Francisco 5 I6 Battery, San Francisco 31 Geary St., San Francisco 339 Bush St., San Francisco San Francisco, San Francisco co San Francisco, San Francisco co 26 Stockton St., San Francisco Woodside, San Mateo co Oakland, Alameda co N. E. cor. Parker and Santa Rosa Aves., San Francisco Haywards, Alameda co Old San Jose Road near Industrial School, San Francisco San Bernardino, San Bernardino co Petaluma, Sonoma co Orange, Los Angeles co 1537 Broadway, San Francisco Petaluma, Sonoma co

S. E. cor. Twenty-first and Alabama Sts., San Francisco San Francisco, San Francisco co 2 I I O'Farrell St., San Francisco Selma, Fresno co Temescal, San Bernardina co Florence, Los Angeles co Sacramento, Sacramento co 126 Sixth St., San Francisco San Jose, Santa Clara co Sanders, Fresno co I ${ }_{03}$ Shotwill, San Francisco 105 Sixth St., San Francisco 607 Sansome St., San Francisco Santa Barbara, Santa Barbara co Arroyo Grande, San Luis Obisco 25 Post St., San Francisco New Castle, Placer co 317 Washington St., San Francisco San Francisco, San Francisco co 
Spence, John $n$.

Stengel, Louis J.

Stratton, W. A. T. $f$

Strong, WV. R., \& Co. n

Sweeney J. P., \& Co. n

Thomas, Milton $n$.

Tisch, D. $n$. . .

Tobin, M. $f$.

Trumbull, R. J.

Trumbull, R. J., \& Co. $f$

Vincent, F. $f$

Vincent, Sevin \& Co. ,

Vowinkel, Henry $f$

Weinhold, Louis $f$

Weinhold, Rufus "

Wellington, B. F. n

Welspiel, Jacob $f$.

Welspiel, W., \& Co. $f$.

Whiting, L. P. $n$.

Wolff, Bernhard $f$

Wolleson, M. $n$.
Santa Barbara, Santa Barbara co

Los Angeles, Los Angeles co

Petaluma, Sonoma co

Sacramento, Sacramento co

$t 09$ Davis St., San Francisco

Los Angeles, Los Angeles co

Oakland, Alameda co

Napa City, Napa co

San Rafael, Marin co

19 Sansome St., San Francisco

32 I Montgomery Ave., San Francisco

607 Sansome St., San Francisco

965 Mission St., San Francisco

Los Angeles, Los Angeles co

Los Angeles, Los Angeles co

425 Washington St., San Francisco

I2 I Sutter St., San Francisco

1 I 7 Sutter St., San Francisco

Milford, Lassen co

107.Fourth St., San Francisco

Calistoga, Napa co

\section{COLORADO.}

Barteldes, Hartig \& Co. $n$.

Bell, Dr. J. $f$

Bird, T. E. $f$

Braun \& Satterwait $n$.

Clark, William $f$.

Cornforth, B. $n$.

Cossea, E. R. n

Gallup, Charlotte R. $f$.

Grimes, D. S., \& Co. n

Hoag, A. N. $f$.

Latshaw, Mrs. Ida M. $f$

Leavy, John $f$

Lee, Henry $n$

Maxwell, C. A. $n$.

Rushmore, H., \& Son $f$

scott, George $n$
205 Fifteenth St., Denver

Colorado Springs, El Paso co

Denver, Arapahoe co

Denver, Arapahoe co

Colorado Springs, El Paso co

Denver, Arapahoe co

Colorado Springs, El Paso co

Denver, Arapahoe co

Denver, Arapahoe co

Fort Collins, Larimer co

Pueblo Floral Conservatory, Pueblo co

Greeley, Weld co

383 Wazee St., Denver

Boulder, Boulder co

Denver, Arapahoe co

367 Blake St., Denver 


\section{CONNECTICUT.}

Acker, Lafayette $n$

Allen, Charles $n$.

Atkins, Isaac $f$.

Augur, Charles P. ,

Austin, T. C. $n$

Barnes, Thomas E. $f$.

Barrows, A. H. $f$.

Bassett, Lyman $u$

Beebe, H. C., \& Co. $n$.

Bettis, D. M. C. $n$

Bishop, J. E. $n$

Bloch, T. E. $n$

Blush, W. C. $f$.

Bowditch, James H. n.

Brewster \& Burnett $n$.

Brooks, D. W. $f$.

Burnwell, E. E. $n$

Burton, John H. $f$

Butler, F. H. $n$

Cable, Mrs. H. C. $f$

Chamberlain, S. D. $n$.

Clark, Alburtus L. $n$.

Clark, John N. $f$.

Clark, Mrs. M. G.

Cocking, Robert

Coffee, James S. $f$

Comstock, Ferree \& Co. $n$.

Comstock, W. G. $n$

Cone, J. H. \& W. E. n

Cooke, John

Coombs, John $f$.

Crane, Stephen $n$.

Crosby, Gordon \& Co.

Dallachie, A. G. $f$

Dallas, Alexander $f$

Decker, Egbert $n$.

Dickerman, George A. $f$

Duerr, John $f$

Free, R. $f$.

Garlick, William, Jr,

Geduldig, Gustave $f$

Goldly, E. L. $n$.

Griswold, Thomas \& Co. $n$.
Easton, Fairfield co

Hartford, Hartford co

Hazardville, Hartford co

Woodbridge, New Haven co

Suffield, Hartford co

Collinsville, Hartford co

Glastonbury, Hartford co

Clintonville Station, New Haven co

Middletown, Middlesex co

Mansfield Centre, Tolland co

Thomastown, Litch field co

Woodmont, New Haven co

246 Fairfield Ave., Bridgeport, Fairfield co

Pomfret, Windham co

Norwich, New London co

- Hartford, Hartford co

P. O. Box 137, New Haven

New Haven, New Haven co

Woodstock, Windham co

Danbury, Fairfield co

Hartford, Hartford co

Orange, New Haven co

Old Saybrook, Middlesex co

Willimantic, Windham co

Danbury, Fairfield co

Norwich, New London co

Wethersfield, Hartford co

East Hartford, Hartford co

Hartford, Hartford co

Greenwich, Fairfield co

Hartford, Hartford co

Norwich, New London co

Danbury, Fairfield co

Plantsville, Hartford co

Waterbury, New Haven co

Wethersfield, Hartford co

New Haven, New Haven co

Meridan, New Haven co

Stamford, Fairfield co

Meriden, New Haven co

Norwich, New London co

Stamford, Fairfield co

South Wethersfield, Hartford co 
Guigue, Peter L. $f$ Hale, G. H. \& J. H. n Hamilton, C. A. Hamilton, William $n$ Hanford, R. H. $f$. Hardy, Alfred $f$. Hawley, R. D. $n$ Hippard, I. H. S. $f$ Holmes, William Horan, James $f$ Houghtail, W. $n$. Hoyt's Sons, Stephen Jennings, E. B. $f$. Jennings, James, \& Son $f$ Jewell, Pliney $f$. Johnson, Robbins \& Co. $n$. Kirtland, H. S. $n$. Lewis, J. A. n Lines, C. P. $n$ Lockwood, J. L. $f$. Loomis, Burdett $f$ Maltman, Thomas Marchant, Robert Mead, Alexander Meggat, William Merwin, Charles D. $n$. McCabe, M. J. $n$. McClunie Brothers McCrone, Robert McKenna, Patrick $f$ McLean, J. B. $f$. McRonald, Thomas $f$. Olmstead, Thomas W. $f$ Park, James W. $f$. Payne, F. W. $f$. Peck, B. H. " Peck, J. M. $f$

Pierson, Andrew $f$ Pilling, Reuben, \& Son $f$ Platt, Frank S. $f$.

Platt, N. S. $n$

Plumb, J. L. $f$

Porter, Charles $\mathrm{H}, f$

Precht, Henry $f$. Reck, John C. $f$. Rehbein, F. N. $f$. Revell, John Richardson, F. W. Sage, F. S., \& Son
Norwalk, Fairfield co South Glastonbury, Hartford co Meriden, New Haven co Norwich, New London co Norwalk, Fairfield co Farmington, Hartford co Hartford, Hartford co Plantsville, New Haven co Birmingham, New Haven co Bridgeport, Fairfield co Seymour, New Haven co New Canaan, Fairfield co Southport, Fairfield co Preston, New London co Hartford Wethersfield, Hartford co Yalesville, New Haven co Willimantic, Windham co 23 Beers St., New Haven Stamford, Fairfield co Hartford, Hartford co Hartford, Hartford co Hartford, Hartford co Greenwich, Fairfield co IVethersfield, Hartford co Milford, New Haven co West Cornwall, Litchfield co Hartford, Hartford co Thompsonville, Hartford co Wallingford, New Haven co Simsbury, Hartford co Hartford, Hartford co Stamford, Fairfield co New Haven, New Haven co 12 Village St., Hartford Cheshire, New Haven co Bristol, Hartford co Cromwell, Middlesex co Danielsonville, Windham co 396 State St., New Haven, New Haven co

Chesire, New Haven co Litchfield, Litchfield co South Woodstock, Windham co Waterbury, New Haven co Bridgeport, Fairfield co New Haven, New Haven co Norwich, New London co East Hartford, Hartford co Wethersfield, Hartford co 
Saunders, David

Scott, George \& R. E. $f$

Seymour, Ira $n$

Seymour, George

Smith, C. A. n

Smith, H. H. $n$

Smith, John H. $f$.

Spalding, John $f$.

Spear, D. A. $f$

Stedman, George C

Storck, P. H. $f$

Swan, Clarence $n$

Terry, W. A. $f$

Thornily, J. $f$

Thurber, W. R. $f$.

Trumbull, Hugh $f$

Veitch, Archibald $n$

Veitch, Robert, \& Son $n$

Wakeman, H. B. $n$

Wakeman, T. B. $n$

Waterbury, G. H., \& Sons

Wheeler, M. G. $f$.

White, John, Jr. $f$

White, Luman $f$.

Whiting, A. $f$

Wilskey, Herman

Woodruff, S. D. $n$

Young, Charles $n$.

Young, George $n$.
Whitneyville, New Haven co

New Britain, Hartford co

Norwalk, Fairfield co

Norwalk, Fairfield co

Putnam, Windham co

West Haven, New Haven co

Norwalk, Fairfield co

New London, New London co

Hartford, Hartford co

Middletown, Middlesex co

693 Broad St, , Meriden

Bridgeport, Fairfield co

Bristol, Hartford co

New Britain, Hartford co

Brooklyn, Windham co

Hartford, Hartford co

New Haven, New Haven co

428 Chapel St., New Haven, New

Haven co

Westport, Fairfield co

Westport, Fairfield co

Stamford, Fairfield co

Winsted, Litchfield co

Hartford, Hartford co

Winsted, Litchfield co

Hartford, Hartford co

Beau Hill, Norwich P. O., New London co

Orange, New Haven co

Norwich, New London co

Norwich, New London co

\section{DAKOTA.}

Brand, Charles $n$.

Brockman, W. L. $n$

Carl, James $n$.

Cowdry, G. W. $n$.

Dakota Home Nursery

Fuller, E. M. $f$.

Smith, A. B. $n$.

Williams, W. H. n
Big Stone City, Grant co

- Huron, Beadle co

- Deadwood, Lawrence co

Valley City, Barnes co

Running Water, Bon Homme co

Bismarck, Burleigh co

Parker, Turner co

Crook City, Lawrence co 


\section{DELAWARE.}

Anthony, J. IV. $n$

Baylis, L. E. $f$

Boggs, C. $n$.

Brown, J. L. $f$

Carter, E. J. ,

Chalk, J. $f$.

Clayton, H. $n$

Cochran, E. R. n

Coles, Walter $f$.

Dougherty, T. $f$.

Grier, T. $f$

Hargadine, R. W. n

Hayden, M. $f$

Hopkins, C.

Hudson, A. L. $n$.

Humphreys, E. $f$.

Johnson, R. S. $n$.

Landers, J., \& Son $f$

Lapham \& Anthony n

Myer, D. S. $n$

Peters, R. $f$.

Phillips, W. R. n.

Polk \& Hyatt $n$

Pullen, A. $n$.

Scout, A. $n$

Shockley, S. W. n

Stockwell, J. B. $n$

Sutton, R. $f$.

Vandenburg, J. H. n

Zimmerman, H. O. R. n

\author{
Smyrna, Kent co \\ IVilmington, New Castle co \\ Moorton, Kent co \\ Wilmington, New Castle co \\ Willow Grove, Kent co \\ Wilmington, New Castle co \\ Mt. Pleasant, New Castle co \\ Middletown, New Castle co \\ Claymont, New Castle co \\ IVilmington, New Castle co \\ IVilmington, New Castle co \\ Felton, Kent co \\ Wilmington, New Castle co \\ Milford, Kent co \\ Clayton, Kent co \\ Wilmington, New Castle co \\ Stockley, Sussex co \\ New Castle, New Castle co \\ Clayton, Kent co \\ Bridgeville, Sussex co \\ Wilmington, New Castle co \\ Milford, Kent co \\ Odessa, New Castle co \\ Milford, Kent co \\ Smyrna Landing, Kent co \\ Lewes, Sussex co \\ Moorton, Kent co \\ New Castle, New Castle co \\ Bridgeville, Sussex co \\ Georgetown, Sussex co
}

\section{DISTRICT OF COLUMBIA.}

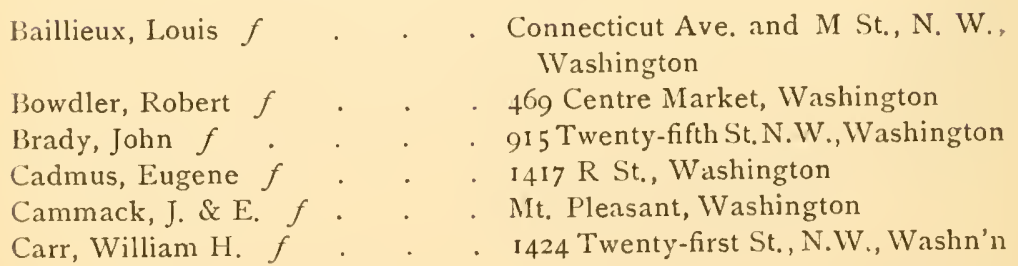


Coleman, William $f$. Diedrich Bros, $f$.

Douglass, Mrs. John $f$.

Esch, Michael $f$.

Field, George, \& Bro. $f$

Freeman, Joseph R. $f$.

Furmage, Mary $f$.

Garden, Alexander $f$

Glorious, George $f$

Huster, Jacob $f$

McQueeney, William, \& Bro.

Miller, Edwin $f$.

Miller, J. M., \& Co. $f$.

Minich, B. $n$

Noll, Simon $f$

Parker, John A. $f$

Pierce, James H. $f$

Saul, John $f$.

Saunders, David I. $f$

Schickler, Christian $f$.

Schmid, Louis, \& Sons

Schultz, Louis $f$.

Small, John H., \& Sons $f$

Strauss, C., \& Co. $f$

Studer, Nicholas $f$

Sivindells, Simeon $f$

Waters, J. J. $f$

Wolter, William $f$
I2 I9 New York Ave., N.W., Washn'n I 227 F St., N. W., Washington 7 I 9 Fourteenth St., N. W. ,Washington I98 Centre Market, Washington Lincoln Ave., Washington Thirteenth \& ESt., N. W., Washington 509 O St., N. W., Washington 44I Centre Market, Washington I I 2 Seventh St., N. W., Washington Brentwood Road, Washington Boundary and Champlain Ave., Washington Bunker Hill Road, Washington 607 Centre Market, Washington Washington

439 Centre Market, Washington I6I6 Nineteenth St. N. W. ,Washington 45 Eastern Market, IVashington 62 I Seventh St., N. W., Washington Pomeroy, N. W., Washington I 355 E St., S. E., Washington 317 Twelfth St., N. W., Washington 609 D St., N. W., Washington Fourteenth and G St., Georgetown 249 Centre Market, Washington 42 I Centre Market, Washington 33 I2 S St., N. W., Washington I 8 I 7 E St., N. W., Washington Fourteenth St. Road, Washington

\section{FLORIDA.}

Adams, C. G. $n$ Beach, A. J., \& Son Codrington, C. $n$. Connolly, D. M. $f$ Cross, J

I)ale, William $f$.

Farrel, Edw. E. $n$ Foster, J. H

Hardee, L. A. n.

Hart, J. E. $n$

Hawkins, W. W., \& Co. n . Helm, J. M., \& Son n. Puetz, Arnold $f$ Styles, W. F.

Whitney, C. J. N.
- Sorrento, Orange co

Palatka, Putnam co

De Land, Volusia co

Jacksonville, Duval co

Liverpool, Manatee co

Jacksonville, Duval co

Valdo, Alachua co

Tangerine, Orange co

Jacksonville, Duval co Jacksonville, Duval co

Lake George, Putnam co

Manatee, Manatee co

Jacksonville, Duval co

Jacksonville, Duval co

Lake City, Columbia co 


\section{GEORGIA.}

Alexander, J. H. n

Berkmans, P. J. n

Blackshear, T. E.

Cole, M., \& Co. $n$

Duncan, T. M. $n$.

Guild, L. A. $\quad x$

Hape, Samuel $n$

Harman, J. C. $n$

Johnson, M. W., \& Co. n

Jones, W. B., \& Sons n

Kiessling, G. $f$

Lambert, A. n

Mackondorff, Edward

Nelson, William K. $n$

Oelschig \& Moyer $f$

Page, T. S. n

Remsen \& Wright $n$

Robinson, WV. P. n

Rumph, S. H. $n$.

Sanford, H. H. $n$.

Sargent, E. M. $n$.

Searcy, IV. H. n

Seiler, Charles $f$

Thompson, IV. IV. $n$

Wachendorff, Edw. n .

Woodruff, C. N., \& Co. $f$

Woodruff, D. B. $n$
Augusta, Richmond co

Augusta, Richmond co

Thomasville, Thomas co

Atlanta, Fulton co

Dallas, Paulding co

Atlanta, Fulton co

Hapeville, Fulton co

Tennille, Washington co

- Atlanta, Fulton co

Herndon, Burke co

Savannah, Chatham co

Atlanta, Fulton co

Atlanta, Fulton co

Augusta, Richmond co

Savannah, Chatham co

Thomasville, Thomas co

Augusta, Richmond co

Atlanta, Fulton co

Marshallville, Macon co

Thomasville, Thomas co

Macon, Bibb co

Griffin, Spalding co

Savannah, Chatham co

Smithville, Lee co

Atlanta, Fulton co

22 Forsyth Road, Macon, Bibb co

Macon, Bibb co

\section{IDAHO.}

Morse, C. W. n

- Boisé City, Ada co

\section{ILLINOIS.}

Aldrich, H. A. $f$. . . Neoga, Cumberland co

Allen, W. D. $f$. . . Io $f$ State St., Chicago 
Alneer Bros, $n$

"American Seed Co." $n$

Anderson, James $H$. $f$.

Anslinger, Martin $f$.

Anthony, Joseph T. $f$.

Armbrush, Nicholas $f$.

Arnold, B. F. $n$.

Augustine, Henry, \& Co. n

Austin \& Co. n .

Bailey, A. H. $u$.

Bailey, William S. $f$

Bailey \& Hanford $n$

Baldwin, Isaac $n$.

Baller, F. A. $f$.

Bargholz, F. $f$.

Barnes, Ambrose B. " .

Bass, James $\mathrm{H}$. n

Beal, L. N. $n$

Belden, J. B. ,

Benthey, Frank F. $f$.

Benton, D. C., \& Co. $n$

Bloomington Nursery Co. $f$.

Blumenscheim, Adam $f$

Boettner, Herman $f$

Boneham, Samuel $n$

Briggs Bros. \& Co. n

Brigham, H. H. n

Bristol \& Gale $n$.

Brooks, F. W. $f$.

Brown, George W. n

Brubach, John G. $n$

Brundage, A. J. $n$

Bryant, Arthur, Jr. $n$.

Buckbee, H. W. $n$.

Buckholz, Charles $f$

Burdich, W. A., \& Co. $n$

Butterworth, T. $n$.

Caldwell, W. B. $n$

Canfield, Frederick P. $f$

Capps, J., \& Son n

Carbon, Joseph $f$.

Cation, William $f$.

Chandler, W. D. $f$

Charlesworth, Samuel

"Chicago Floricultural Co." $f$

Clemens, Peter $f$.

Cole Bros. $f$.

Coleburg, N. P. $f$.

Congdon, Ira $Z$. $n$

Conrad, Andrew $f$
Rockford, Winnebago co

I98 Market St., Chicago

Havelock P. O., Cook co

3545 Wabash Ave., Chicago

I 3 Thirty-eighth St., Chicago

Oak Park, Cook co

Galesburgh, Knox co

Normal, McLean co

Downer's Grove, Du Page co

Rantoul, Champaign co

Evanston, Cook co

Makanda, Jackson, co

Jacksonville, Morgan co

Bloomington, McLean co

Graceland, Cook co

46 Lake St., Chicago

Canton, Fulton co

Mt. Vernon, Jefferson co

Richmond, McHenry co

175 Wabash Ave., Chicago

Quincy, Adams co

Bloomington, McLean co

29 Io Cottage Grove Ave.,

2 I 5 North Clark St., Chicago

Oreana, Macon co

97 Clark St., Chicago

Hopedale, Tazewell co

30 South Canal St., Chicago

4525 Lake Ave., Chicago

46 La Salle St., Chicago

Princeton, Bureau co

P. O. Box 18 , Wing, Livingstone co

Princeton, Bureau co

Rockford, Winnebago co

434 1/2 North Clark St., Chicago

Rockford, Winnebago co

Quincy, Adams co

Paris, Edgar co

Springfield, Sangamon co

Mt. Pulaski, Logan co

Des Plaines, Cook co

Peoria, Peoria co

28I Wabash Ave., Chicago

3445 State St., Chicago

3800 Grand Boulevard, Chicago

4 Centre Ave., Chicago

Peoria, Peoria co

Princeton, Bureau co

Onarga, Iroquois co

$3^{8} 3$ North Clark St., Chicago 
Corbey, Thomas J. / .

Cotta, J. V. n

Craig, Joseph C. f

Cultra, R. B. n

Daube, F. $f$.

Dennis, C. N. n

Dennis \& Bullock $n$

De Rimmer, Thomas

Desmond, IVilliain $n$

Dickinson, Albert $n$

Dilger, F. P. $f$.

Donaldson, Mrs. Mary D. $f$

Donavan, R. J. $f$.

Douglass, Robert, \& Son $n$.

Douglass, T. J. $f$.

Doyle, Michael $n$.

Dresel, August $f$.

Dreuth, Henry $f$.

Duerson, A. L. $f$.

Dunning, D. S., \& Sons $n$

Eirle, P., \& Sons $n$

Ellsworth, Lewis $n$

Ewell, J. F. $n$

Farrell, James $f$

Felke, John $f$.

Fenity, F. C. n

Fischer, Frederick

Forest Glen Floral Co., M. E. Page,

Proprietor $f$

Forster, John $f$

Foster, A. $n$.

Foster, E. E. $f$

Frank, Conard E. n

Franks, Thomas $f$

French, R. H. $f$.

Fry Bros. $n$.

Fuller, Daniel $\mathrm{B}$.

Furman, A. D, $n$.

Galusha, O. B. $n$.

Gardner, W. H. f

Garrett, G. IV. $n$.

Garrison, J. H. n .

Gay, Charles V. n

Genteman, William $f$.

Gescheidle, Henry $f$

Giddings, A. H. $f$

Gillette, H. n

Goode, John $f$

Greb, John $f$.

Gregory Bros.
938 West Madison Ave., Chicago

Lanark, Carroll co

66 Washington St., Chicago

Onarga, Iroquois co

266 Milwaukee Ave., Chicago

Hamilton, Hancock co

Eureka, Woodford co

2720 Fifth Ave., Chicago

Kewannee, Henry co

115 Kinzie St., Chicago

266 Milwaukee Ave., Chicago

128 South Halsted St., Chicago

Havelock P. O., Cook co

W'aukegan, Lake co

Langley Ave. \& Fortieth St., Chicago

Springfield, Sangamon co

656 Clybourn Ave., Chicago

560 Twenty-eighth St., Chicago

Galesburgh, Knox co

Jefferson, Cook co

Cobden, Union co

Napierville, Du Page co

Auburn, Sangamon co

165 IVabash Ave., Chicago

268 Mohawk Ave., Chicago

Jerseyville, Jersey co

Evanston near DiverslyAve., Chicago

209 Wabash Ave., Chicago

P. O. Box 392, Evanston, Cook co

South Evanston, Cook co

Box 582, Jacksonville, Lake co

Bloomington, McLean co

Champaign, Champaign co

Libertyville, Lake co

Alton, Madison co

3759 Cottage Grove Ave., Chicago

Normal, McLean co

Morris, Grundy co

242 Wabash Ave., Chicago

Roscoe, IVinnebago co

Greenwood, McHenry co

Camp Point, Adams co

Quincy, Adams co

I63 Wilmot Ave., Chicago

Danville, Vermillion co

Highland Park, Mason co

Hyde Park, Cook co

Mendota, La Salle co

Bloomington, McLean co 
Crullett \& Berningham $f$ Hackman, H. S. $n$ Hall, S. E. $n$ Hammes, Henry $f$ Hansen, Henry $f$. Harms, A. J. $f$ Harris, H. P. $n$. Hart, Alfred $n$ Hartwig, Charles $f$ Hathaway, E. C. $n$ Heinl, Joseph $n$. Hill, David $n$ Hinckley, James $n$ Hobbs \& Co. $f$ Hoff, O. W. $n$ Hughes, H. R. $f$. Johnson, William H. n Keim, Cyrus $\mathrm{H}$. $n$ Keith, S. L. $n$ Kennicott, Joseph $n$. Kersting, Theodore $f$. King, James $f$

Kirchgraver, J., \& Son $n$ Kirchhoff, F., \& Son $f$. Klaus, Delker $n$.. Klimmer, Joseph $f$ Knoddle Bros. $n$. Knowlton, 1. S. $n$. Kreitling, Oscar $f$ Kreitling, WValter $f$ Krick, George $f$.

Krohn, Paul

Krueger, Charles F. $f$. Krug, J. $f$ Larson, N. P. $f$ Lawson, F. B. $f$. Leesby, Robert $n$. Lewis, R. J. n Lindeman, Charles $n$ Malsh, Moses $f$. Manahan, Frank T. $f$. Mann, F. D. n Marson, T., \& Sons $n$. Merritt, Mrs. J. E. $f$. Niller \& Hunt $f$

Miller, Lewis, \& Co. $f$. Minkler, S. G, $n$.
Lincoln, Logan co

Peru, La Salle co

Cherry Valley, Winnebago co 325 Division Ave., ChicagoHavelock P. O., Cook co Morton Grove, Cook co Champaign, Champaign co Vermont, Fulton co Wright's Grove P. O., Cook co ()ttawa, La Salle co Jacksonville, Morgan co Dundee, Kane co Rockford, Winnebago co 3112 Indiana Ave., Chicago Wataga, Knox co 103 Aberdeen St., Chicago Ivesdale, Champaign co Mt. Carroll, Carroll co Palatine, Cook co Arlington Heights, Cook co 177 Henry Ave., Chicago 78 Wabash Ave., Chicago Mattoon, Coles co 50 I West North Ave., Chicago 475 Blue Island Ave., Chicago I66 West Madison Ave., Chicago Irving, Montgomery co Byron, Ogle co 749 South Halsted Ave., Chicago 2 I9 Wabash Ave., Chicago 65 State St., and 235 Ontario Ave., Chicago

219 Clark St., and 26I Chicago Ave., Chicago

73 Washington Ave., Chicago Alton, Madison co 265 Washington Ave., Chicago 6I Washington Ave., Chicago Havelock P. O., Cook co 337 Racine Ave., Chicago Wright's Grove P. O., Cook co 206 South Halsted Ave., Chicago 578 Vest Madison Ave., Chicago Gilman, Iroquois co Morgan Park, Cook co 65 Lake St., Chicago Wright's Grove P. O., and Chicago,

Cook co

Peoria, Peoria co Oswego, Kendall co 
Moore, S. A. $f$

Morgan, Aaron W. $f$.

Morris, H. M.

Muir, Samuel

Mulder, Car

Munz, John $x$

Murdock, R. R. $n$

Murray, J. C. $f$

Musgrove \& Mills $n$

McCarthy, Charles $f$

McKinney, E. P. n

McKinstry, Byron N. n

McPheron, J. A. $n$

McWharter, T. $n$.

Neltnor, J. C. $n$

Newitt, William G. $f$

Nilson, John $f$

Noe, Smith $n$

Nourse, William A. $n$.

Olds, Warren $n$

Orr, Arthur W. $f$.

Page, M. E., \& Co. $f$

Painter, H. C. $n$. .

Palmer, S. E. \& Co. $n$.

Peck, D. E. $n$

"Peoria Home Nursery" $f$.

Pervis, Robert $f$.

Peters, William $f$.

Peterson, P. $n$.

Peterson, P. S. $n$.

Peterson, R. S. $n$.

Petingill, John A. n

Phelps, H. L. $f$

Philips, W. R. $n$.

Phoenix Bros. \& Emerson $n$

Piper, D. J. $n$

Prescott, C. W. $n$.

Pullman, S. M. $f$.

Rederluigo, Henry

Rees, Richard $n$.

Reeves, J. B. $n$.

Reid, W. J. n

Reissig, Charles

Richmond Bros.

Ricker, E. A., \& Co. n

Rike, L. A. n

Rimmer, Thomas D. $f$

Rindham, J. n

Riordan, John $n$

Rivers, Joseph $f$.
Leroy, McLean co

Danville, Vermillion co

Rantoul, Champaign co

3530 Michigan Ave., Chicago

87o West Lake Ave., Chicago

Odell, Livingston co

Paxton, Ford co

Peoria, Peoria co

Rozetta, Henderson co

Webster, cor. Sheffield Ave., Chicago

Lacon, Marshall co

Grant Park, Kankakee co

Carrollton, Greene co

Aledo, Mercer co

Turner Junction, Du Page co

3800 Grand Boulevard, Chicago

Graceland, Cook co

Beverly, Adams co

Moline, Rock Island co

Albany, Whiteside co

Sandwich, De Kalb co

203 Wabash Ave., Chicago

Effingham, Effingham co

Bloomington, McLean co

Marengo, McHenry co

I7or Perry St., Peoria, Peoria co

Havelock P. O., Cook co

78 Canalport Ave., Chicago

Jefferson, Cook co

I64 La Salle St., Chicago

Havelock P. O., Cook co

Bunker Hill, Macoupin co

Springfield, Sangamon co

Milford, Iroquois co

Bloomington, McLean co

Forreston, Ogle co

Marengo, McHenry co

Pullman, Cook co

I I Rosebud Ave., Chicago

Aurora, Kane co

Shelbyville, Shelby co

Chatsworth, Livingston co

I69 Wabash Ave., Chicago

Geneseo, Henry co

Elgin, Kane co

Leroy, McLean co

2720 Fifth Ave., Chicago

Bloomington, McLean co

Bloomington, McLean co

I 7 Blue Island Ave., Chicago 
Robinson, J. D. $f$.

Robinson, Norman $f$

Root, J. B., \& Co. n

Sanders, E.

Schmidt, F. C. G. $f$

Schroeder, H. $n$

Schwarz \& Heinecke $f$

Scott, D. W.

Semmler, Michael $n$.

Shank Bros. n

Sharpe, William H. $f$.

Shumivay, R. H. $n$

Silliman, Irving C. $f$

Sinnock \& Co. $n$.

Skinner, E. H. $n$.

Small, A. L. $n$.

Smith, Frank B. $f$

Smith, F. H. $n$

Smith, George H. $f$

Smith, IV. H. $f$

Smith \& Co.

Sommers \& IVilkes $f$. Spalding, J. B. \& Co. $f$ Spalding, J. B. \& Co. $f$ Specht, Joseph I. $f$. Stahley, Miss Julia A. $f$ Strubble, Philip $n$ Stuenkel, William $f$ Sulzer, Frederick $f$ Sulzer, Frederick $f$ Summer, Charles H. n Summerville, T. $n$ Then, Anton $f$

Thrapp, R. B. $n$.

Tilton Bros. $n$

Tull, John R. $n$

Twiss, George W.

Unverzogt, Louis $n$

Vanovan, E. $n$.

Vaughn, J. C. $f$.

Vawter, A. T. $n$

Vogil, Jacob $f$

Watson, W. A. $n$.

Webster, Jabez $n$.

Weeks, John B. $f$

Weiland, M. $f$

Weinhoeber \& Tonne $f$

"Western Seed Co." $n$

Westgate, C. A. $n$

Wharton, H. E. $n$
Bloomington, McLean co

862 West Lake Ave., Chicago

Rockford, Winnebago co

Chicago, Cook co

I04 Seminary Ave., Chicago

Bloomington, McLean co

66. Halsted Ave., Chicago

Galena, Jo Daviess co

Belleville, St. Clair co

Clayton, Adams co

${ }_{3} S_{3}$ N. Clark Ave., Chicago

Rockford, Winnebago co

553 West Madison Ave., Chicago

Quincy, Adams co

Rockford, Winnebago co

Kankakee, Kankakee co

Danville, Vermillion co

Bement, Pratt co

255 West Madison Ave., Chicago

Aurora, Kane co

Englewood, Cook co

Quincy, Adạms co

Riverton, Sangamon co

Springfield, Sangamon co

I 74 North Clark Ave., Chicago

55 Washington Boulevard, Chicago

Naperville, Du Page co

Chicago, Cook co

Graceland, Cook co

Ravenswood, Cook co

Polo, Ogle co

Bunker Hill, Macoupin co

830 Lanabee St., Chicago

Tallula, Menard co

Aurora, Kane co

Fruitland, Hancock co

Rockford, Winnebago co

Springfield, Sangamon co

Naperville, Du Page co

42 La Salle St., Chicago

Macomb, McDonough co

225 I Cottage Grove Ave., Chicago

Normal, McLean co

Centralia, Marion co

59 Lake Ave., Chicago

Evanston, Cook co

Wright's Grove P. O., Cook co

33 Michigan Ave., Chicago

Peotone, Will co

Payson, Adams co 
Wheeler, L. P. $n$.

Whitcomb \& Maxfield $n$

Whitcombe, S. M. $n$

Whitney, A. R. $f$.

Wienhoeber, Ernest $f$.

Wienhoeber, Otto $f$

Wilson, James $f$.

Williams, H. W. n

Williams, H. W. n

Williams, H. W., \& Sons $f$

Williams, W. H. n

Wood, H. A. $f$

Woodburn, J. H. $f$

Wright, Grove $n$.

Wyckoff, Samuel $n$

Yackley, M. $n$.

Zender, Adam $f$.
Quincy, Adams co

Albany, Whiteside co

Albany, Whiteside co

Franklin Grove, Lee co

425 Elm Ave., Chicago

38 I North Clark St., Chicago

Palatine, Cook co

Galesburgh, Knox co

Peoria, Peoria co

Batavia, Kane co

Pittsfield, Pike co

Petersburgh, Menard co

Sterling, Whiteside co

Rock Falls, Whiteside co

Woodson, Morgan co

Pittsfield, Pike co

Havelock P. O., Cook co

\section{INDIAN TERRITORY.}

\section{INDIANA.}

Albertson \& Co., $n$

Albertson \& Hobbs, $n$

Allen, Wm. Z. $f$.

Armentrout, J. N., $n$

Baldwin, Wm. $n$

Baxter, Mrs. Kate M. $f$

Beach \& Co., $f$

Bertermann Bros., $f$

Bettmann, John G. $f$

Bird, John $n$

Blackman, Wm. $n$

Bodge, J. S. $n$

Bowen, Geo. D. $n$

Bowman, Robt. $n$

Brown \& Bro. $n$.

Brown, Robt. P. $n$

Buckingham, B. F. n.
Canton, Washington co

Bridgeport, Marion co

Monrovia, Morgan co

Frankfort, Clinton co

Marion, Grant co

Mt. Vernon, Posey co

Richmond, Wayne co

Indianapolis, Marion co

New Albany, Floyd co

Raysville, Henry co

Evansville, Vanderburgh co

La Porte, La Porte co

Evansville, Vanderburgh co

Pennville P. O., Jay co

Warrington, Hancock co

Greenfield, Hancock co

Union City, Randolph co 
Burnett, S. $n$

Carmody, J. D., \& Co. $f$

Case, L. B. $f$

Chandler, A. G., \& Co. $n$.

Clarke, H. M. $n$

Cole, R. S. $n$

Conde, H. T.

Cooper, Mary E. $f$

Cowing, Granville, \& Son $n$

Cox, D. W. $f$

Crist, R. G. $n$

Davis, J. C. $n$

Dean, Argus $n$

Dorner, F. $f$.

Doswell, Geo. IV. $f$

Dumont, S. M. $f$.

Dunk, Edgar $f$

Eddy, Burton $n$

Everett, J. C. $n$

Fisher, D. A. ,

Fohl, Bernie A. $f$.

Foote, J. A. $f$

Glenn \& Ruddick $n$

Gould, Dr. Vernor $n$

Graham, David A. $f$

Graham, Henry $f$

Graham, J. K. $n$.

Gregg \& Son $n$

Griess, John Jr. n

Haines, G. W. $n$.

Halleck, James $n$

Hammond, Valentine $n$

Haney, Robt. $n$.

Heinl, John G. $f$.

Heinl, Lawrence

Henby, J. K. $n$.

Hilker, Henry $f$.

Hill \& Co. $f$.

Holland, William n

Holmes, L. $n$

Hubbard, W. J. n

Humfeld, S., Jr. $f$.

Kidd, W. J. $f$

Lawrence, Ellis $n$

Lewis, Thomas $f$.

Markey, E. J. $f$.

Mason, Robert $f$.

Mendenhall, J. F., \& Co. $n$.

Merrifield, George C. $n$

Miller \& Hunt $f$.
Vincennes, Knox co

Evansville, Vanderburgh co

Richmond, Wayne co

Indianapolis, Marion co

Covington, Fountain co

Indianapolis, Marion co

Indianapolis, Marion co

Columbus, Bartholomew co

Muncie, Delaware co

Crawfordsville, Montgomery co

New Market, Montgomery co

Bethlehem, Clark co

Otto, Clark co

Lafayette, Tippecanoe co

Ft. Wayne, Allen co

Vevay, Switzerland co

Evansville, Vanderburgh co

Wheeler, White co

Lima, Lagrange co

Denver, Miami co

Peru, Miami co

Terre Haute, Vigo co

Columbus, Bartholomew co

Rochester, Fulton co

Rockville, Parke co

Terre Haute, Vigo co

New Albany, Floyd co

Pennville P. O., Jay co

Evansville, Vanderburgh co

Big Spring, Boone co

Lake Village, Newton co

Millwood, Kosciusko co

Terre Haute, Vigo co

Terre Haute, Vigo co

Terre Haute, Vigo co

Greenfield, Hancock co

Indianapolis, Marion co

Richmond, Wayne co

Plymouth, Marshall co

Medora, Jackson co

Columbus, Bartholomew co

Cochran, Dearborn co

Logansport, Cass co

Plainfield, Hendricks co

Indianapolis, Marion co

Fort Wayne, Allen co

Madison, Jefferson co

Indianapolis, Marion co

Mishawaka, St. Joseph co

Terre Haute, Vigo co 
Millhouse, J. V., \& Son $n_{\text {, }}$.

Moore, George $f$.

Moyer, George N. $n$.

McMasters, William $f$.

Neyhard, L. C. $n$.

Parry, Thomas $n$.

Parsons, John $f$

Patterson \& Tomlinson $n$

Pegden, William $f$.

Phelps, J. C. $n$

Preble, A. C. $n$

Ragan, IW. A. $n$

Redding, Thomas B. n

Sanders, James $n$.

Shopp, H. L. $n$

Simpson \& Hogue $n$

Sleeper, Alonzo D. $n$.

Sleeper, William M. $n$.

Smith, G. $f$.

Stansil, H. W. $n$.

Stine, H. S. $n$

Street, I. F. $n$

Strumpf, G. W. $n$.

Teas, Eli Y. $n$.

Thompson, John T. $n$

Tinslow, E. V. $n$.

Walker, Francis $n$

Ward, Thomas J. $n$

Weary, John $n$.

Weaver, A. J. $n$.

White, Jesse $n$

Wiggan, A. $f$

Wooden, J. H. $n$.

Wooten, William S. $n$.

Wyeth, E. R. \& J. W. n

Young, James, \& Bros. $n$
Butlerville, Jennings co

Milton, Wayne co

Laketon, Wabash co

Montezuma, Parke co

Mulberry, Clinton co

Lagrange, Lagrange co

Floyd's Knobs, Floyd co

Logansport, Cass co

Marion, Grant co

Westfield, Hamilton co

Somerset, Wabash co

Clayton, Hendricks co

New Castle, Henry co

Westfield, Hamilton co

New Castle, Henry co

Vincennes, Knox co

Oxford, Benton co

Oxford, Benton co

Smithland, Shelby co

Delphi, Carroll co

Burnett's Creek P. O., White co

West Middleton, Howard co

Roanoke, Huntington co

Dunreith, Henry co

Amo, Hendricks co

Petersburgh, Pike co

New Albany, Floyd co

Saint Mary's, Vigo co

Kewanna, Fulton co

Danville, Hendricks co

Plainfield, Hendricks co

Indianapolis, Marion co

Indianapolis, Marion co

Danville, Hendricks co

- Riley, Vigo co

- Greensburgh, Decatur co

\section{IOWA.}

Alexander, James $n$. Baldwin, J. E. $n$. Bancroft, Joseph $n$

Barnard, C. \& F. G. $n$.

Barnes, W. W., \& Son n

Barton \& McGinnis $n$.

\section{Chariton, Lucas co}

Des Moines, Polk co

Cedar Falls, Black Hawk co

Waukon, Allamakee co

- Knoxville, Marion co

Harlan, Shelby co 
Barton, George D. n Bathers, J. R. \& A. $f$.

Beyer, Hugo $n$.

Binning, C. W. $n$.

Blair, R. L. $f$

Black, E. $n$.

Bock Bros. "

Bonham \& Hammond $x$

Bowers, Samuel $f$.

Brackett, G. B. $n$.

Branson, Abner $n$

Brice, A. C. $n$.

Briggs, D. M. $f$

Brown, T. F. $n$

Bruner, D. F. $n$

Bryan, James W., \& Co. $n$.

Burt, N. J., \& Co. n

Burtis, J. A. $f$

Burton, C. W. $n$

Carson, Thomas C. $n$.

Carroll, O. $n$.

Chapin, WV. E. $n$.

Christman \& Healy $n$.

Cole Bros. $n$.

Cooper, Albert $n$.

Cooper, L. A. $n$

Crabb \& Co. $n$

Cripps, J. B. $n$

Crouse, H. $n$

Cummings, G. J. n

Dayton, John P. $f$

Dorr, C. W., \& Co. n .

Ennis \& Patton $n$.

Farrington \& Bros. $n$.

Ferris, S. W. $n$

Filkins, Charles H. $n$.

Foster, S. $n$.

Foster, W. H.

Fowler, Mrs. H. J. $f$

Gardner, Charles F. $n$.

Giddings, A. n

Giddings, Alfred n

Gore, C. W.

Goodrich, Daniel O. $n$.

Graves, A. J. $n$

Grippen, A. L. $f$.

Guilford, W. H. n

Haldeman, F. W. n

Harkett Floral Nursery

Harvey Bros. $n$.
Perry, Dallas co

Clinton, Clinton co

New London, Henry co

Dyersville, Dubuque co

Des Moines, Pulk co

Jesup, Buchanan co

Burlington, Des Moines co

Malvern, Mills co

Cedar Rapids, Linn co

Denmark, Lee co

West Branch, Cedar co

Lenox, Taylor co

Avoca, Pottawattamie co

Newton, Jasper co

Toledo, Tama co

Cedar Rapids, Linn co

Burlington, Des Moines co

Boone, Boone co

Cedar Rapids, Linn co

Iowa City, Johnson co

Boonesborough, Boone co

Des Moines, Polk co

Dubuque, Dubuque co

Pella, Marion co

Coal Creek, Keokuk co

Council Bluffs, Pottawattamie co

Red Oak, Montgomery co

Albion, Marshall co

Montrose, Lee co

Mitchell, Mitchell co

Waukon, Allamakee co

Des Moines, Polk co

Clinton, Clinton co

Bloomfield, Davis co

Bristow, Butler co

Brooklyn, Powesheik co

Muscatine, Muscatine co

Council Bluffs, Pottawattamie co

Waterloo, Black Hawk co

Osage, Mitchell co

Sabula, Jackson co

Sterling, Jackson co

Belle Plain, Benton co

Downey, Cedar co

Ames, Story co

Mason City, Cerro Gordo co

Dubuque, Dubuque co

Avoca, Pottawattamie co

Dubuque, Dubuque co

Indianola, Warren co 
Haviland, A. J. n. Herman, B. D. $f$. Heyne, G. A. n Higley, H. G. $f$. Hilton, George O. n Hoffmeister Bros. n Hoyer, I. R. n Hoyt, Edward $n$. Humphrey, P. D. n Hysham, IV. J. $n$. Jacquith, B. F. $n$. Johnson, W. T. $f$. Kauffman, L. $n$. Kemble, A., \& Co. $\quad$ i Klein, W. H. n Lake, D. S. $n$ Lantz, J. W. n Leonard, David $n$ Light, S. n Livingston, A. W. $n$ Lovett, M. $n$. Mason, T. E. n Means, S. $\dot{\mathrm{E}}$. $f$ Meneray, Mrs. S. E. $n$. Mitchell, J. B. $n$ McClary \& Hitchcock n McClellan \& Moore $n$. McGill, P. $f$. Needles, I. M. $n$. Nichols, O. P. n Oaks, Edwin $n$ Oliver, E. A. $n$ Osborn, C. P. $n$. Page \& Kelsey, $f$. Parks, Samuel $n$. Patton, C. J. n Paxton, S. S. $n$ Pearson, J. H. n. Perkins, J. L. $n$. Pfautz, John M. n Pierce, L. F. $n$ Pilsbry, D. R. $f$ Pitts, T. J. $n$. Platt, John $n$ Porter, Erwin $n$. Prestle, W. H. $f$. Pugsley, M. Reppart, C. $n$ Reynolds, E. C. $n$ Rice, T. R. $u$
Fort Dodge, IVebster co Sigourney, Keokuk co Dubuque, Dubuque co Cedar Rapids, Linn co Keokuk, Lee co Fort Madison, Lee co Boonesborough, Boone co Scotch Grove, Jones co Nevada, Story co Red Oak, Montgomery co Des Moines, Polk co Ottumwa, Wapello co Iowa City, Johnson co Oskaloosa, Mahaska co Baxter, Jasper co Shenandoah, Page co Brooklyn, Powesheik co Burlington, Des Moines co Fairfield, Jefferson co Des Moines, Polk co Elkport, Clayton co Shenandoah, Page co Vinton, Benton co Crescent, Pottawattamie co Cresco P. O., Howard co High Point, Decatur co Batavia, Jefferson co Ainsworth, Washington co Atlantic, Cass co Davenport, Scott co Des Moines, Polk co Davenport, Scott co Fairfax, Linn co Des Moines, Polk co Ottumwa, Wapello co Charles City, Floyd co Ames, Story co Ainsworth, Washington co Little Sioux, Harrison co Lisbon, Linn co Kossuth, Des Moines co Iowa City, Johnson co Nashua, Chickasaw co Colesburgh, Delaware co Delhi, Delaware co Iowa City, Johnson co Woodbine, Harrison co Burlington, Des Moines co Muscatine, Muscatine co Council BIuffs, Pottawattamie co 
Robertson, W. W. f

Russell, J. M. n

Savage, E. W. $f$.

Schafer Bros. $n$

Schee, Nathan, \& Co. "

Shaver, C. J. $f$.

Shirland, J. H. $n$.

Smith, IV. $n$.

Springborn, William $f$.

Stacey, J. E. $n$.

Stewart, W. H. n .

Strohm, H. n

Swaney, John S. n

Temple, J. T. $n$

Terry, H. A. n

Tipton, John H. n

Van Sands, L. J. $n$

Walden, F., \& Co. n

Watrous, C. L. $n$.

Weber, Julius $n$

Weiss \& Mair $n$.

Wells, J. $n$.

Weyland, H.

Wilson, Silas $n$

Wisse, P. $n$.

Woodrow, J. M. $n$

York Nursery Co. $n$

Young, J. M. n
Keokuk, Lee co

Essex, Page co

Iowa Falls, Hardin co

Pella, Marion co

Milo, Warren co

Fredonia, Louisa co

Grinnell, Powesheik co

Sioux City, Woodbury co

Dubuque, Dubuque co

Algona, Kossuth co

Danville, Des Moines co

Iowa City, Johnson co

Marengo, lowa co

Davenport, Scott co

Crescent, Pottawattamie co

Springdale, Cedar co

Odebolt, Sac co

Albion, Marshall co

Des Moines, Polk co

Malcom, Poweshiek co

Council Bluffs, Pottawattamie co

Steamboat Rock, Hardin co

Keokuk, Lee co

Atlantic, Cass co

Alton, Sioux co

Newton, Jasper co

Des Moines, Polk co

Sioux City, Woodbury co

\section{KANSAS.}

Amburn, J. M. $n$.

Baird, J. C. n

Barnes, Reuben C. $f$

Barteldes, F., \& Co. $n$.

Beymer, Mrs. I. C. $n$.

Bidgood, J. W. $n$.

Bowen, J. H. $n$.

Brackett, G. C. $n$.

Brane, C. J.

Bristol, C. E. $n$

Bristol Sisters $f$

Brooks, Mrs. P. $f$.

Brower, E. L. $f$.

Brown, G. B. $n$
Peabody, Marion co

Easton, Leavenworth co

Atchison, Atchison co

Lawrence, Douglas co

Hiawatha, Brown co

Leavenworth, Leavenworth co

Eureka, Greenwood co

Lawrence, Douglas co

Winfield, Cowley co

Fulton, Bourbon co

Topeka, Shawnee co

Lawrence, Douglas co

Clay Centre, Clay co

Guilford, Wilson co 
Cadwallader \& Bro. $n$.

Carter, C. A. $n$. .

Chambers, A. D. $n$.

Chase, Reuben C. $n$.

Chevey, A. A. $n$.

Clothier, E. E. $n$.

Cook, H. A. B. $n$.

Cook, T. F. n .

Cozad, D. W. $n$.

Crandall, R. W. $n$

Cross, John A. $n$.

Cutler, William, \& Sons $n$

Diehl, E. P. $n$

Downs, L. H. $n$.

Eason, Frederick $n$

Ellsworth, A. $n$

Gardner, O. A. $f$

Goodwin, J. n

Gray, O. N. n

Griesa, A. C. $n$

Griesa, A. H. n

Groves, R. J. $n$

Harding, W. M., \& Son $n$

Hart, H. B. n

Hart's Pioneer Nursery $n$

Hawthorne, D. C. $n$

Holdsworth, S. \& Co. n

Holman, E. J. $n$.

Keith, Wilson $n$.

Kern, H. H. n

Ketcham, Oscar $n$

Kroh, G. L. $n$

Latimer, J. W., \& Co. n

Lyne, G. $n$

Mangeldorf Bros. $f$

Mosher, James A. n

Moshiskey, John $n$

Mostellar, G. W., \& Bro. $n$

Myer, E. L.

McCauley, E. P. $f$

McVay, L. C., \& Co. $n$.

Parson's Nursery $n$

Peters, Son \& Taylor $n$

Phillips, P. P. $n$. .

Salmon, J. $n$.

Shaw, C. W. $n$

Sieves, C. T. $n$

Sinith, B. F. n

Sturns, J. H. n

Stewart, Martin

Swift, Harry $f$
Louisburgh, Miami co

Quakervale, Cherokee co

Hartford, Lyon co

Hiawatha, Brown co

Belleville, Republic co

Morantown, Allen co

Blue Mounds, Linn co

Monrovia, Atchison co

La Cygne, Linn co

Newton, Harvey co

Chanute, Neosho co

Junction City, Davis co

Olathe, Johnson co

73 Kansas ave., Topeka, Shawnee co

Leavenworth, Leaven worth co

Hutchinson, Reno co

Belleville, Republic co

Wıllis, Brown co

Jewell, Jewell co

Lawrence, Douglas co

Lawrence, Douglas co

Atchison, Atchison co

Lawrence, Douglas co

Fort Scott, Bourbon co

Fort Scott, Bourbon co

Leavenworth, Leavenworth co

Enterprise, Dickinson co

Leavenworth, Leavenworth co

Topeka, Shawnee co

Tiblow, Wyandotte co

Chetopa, Labette co

Wyandotte, Wyandotte co

Pleasanton, Linn co

Huron, Atchison co

Atchison, Atchison co

Belleville, Republic co

Marysville, Marshall co

Girard, Crawford co

Hutchinson, Reno co

Ellsworth, Ellsworth co

Wetmore, Nemaha co

Parsons, Lebette co

Topeka, Shawnee co

Lawrence, Douglas co

Corning, Nemaha co

Sabetha, Nemaha co

Washington, Washington co

Lawrence, Douglas co

Wichita, Sedgewick co

Quindaro, Wyandotte co

Chapman, Dickinson co 
Taylor, E. A. $n$

Taylor \& Jackson $n$

Treat, H. B.

Truitt, James $n$

Williams, J. L. $u$.

Williams, Lewis $n$

Williams, O. N. $n$

York Nursery Co. n
Beloit, Mitchell co

Grantville, Jefferson co

Atchison, Atchison co

Chanute, Neosho co

Oswego, Labette co

Chetopa, Labette co

Columbus, Cherokee co

Fort Scott, Bourbon co

\section{KENTUCKY.}

Bacon, W. A. $n$

Bell, C. S. $n$.

Bell, W. S. $f$.

Bingham, J. C. $n$.

Central Floral Bazaar $f$

Chambers, S. R. n

Collings, H. \& Co. n

Dietrich, C. P., \& H. C. $n$

Downer \& Bro. $n$.

Downer \& Bro. $n$.

Dunlavy, B. B. $n$.

Estelle, Samuel $f$.

Fall \& Bro. $n$

French, L. G. $n$

Gaar, S. L. $n$

Gage, Lewis, \& Co. $n$.

Garnier, Louis $f$

Gibson, L. $f$.

Greene \& Greene $f$

Greene, Mrs. P. $f$.

Hale, C. P. $n$

Haupt Bros. $f$

Hewitt, Field \& Co. $n$

Hillenmeyer, H. F. $n$.

Hinton, James $f$

Hodgman, F. E. $f$

Honaker, David $n$

Johns, U. E. n

Keller, J. A. $f$

Knight, George $f$.

Lauer, Anton $f$.

McGill \& Truman $n$

McKee, J. A. $n$

McNally, Ed. $f$

Miller \& Garner $n$
Paris, Bourbon co

Lexington, Fayette co

Lexington, Fayette co

Russell, Greenup co

Louisville, Jefferson co

Louisville, Jefferson co

224 West Main St., Louisville

Maysville, Mason co

Elkton, Todd co

Guthrie, Todd co

Pleasant Hill, Mercer co

Owingsville, Bath co

Fulton, Fulton co

Gordonsville, Logan co

Anchorage, Jefferson co

Louisville, Jefferson co

Dayton, Campbell co

Louisville, Jefferson co

4 I 7 th St., Louisville, Jefferson co

Louisville, Jefferson co

Calhoun, McLean co

Louisville, Jefferson co

Louisville, Jefferson co

Lexington, Fayette co

Paris, Bourbon co

Louisville, Jefferson co

Lexington, Fayette co

South Union, Logan co

Lexington, Fayette co

Bowling Green, Warren co

Louisville, Jefferson co

Louisville, Jefferson co

Cynthiana, Harrison co

Anchorage, Jefferson co

Winchester, Clark co 
Morat, Ed. $f$.

Morat, Francis $f$.

Nanz \& Neuner $f$.

Neuner, Charles $f$

Reimers Bros. $f$.

Richardson, WV. A. $f$

Roach, John E. $f$.

Rompeu \& Marret $f$

Ruth, C. D. \& E. D. $n$.

Samuels, William, \& Co.

Schulz, Jacob $f$

Schwarz, Mrs. Mary $f$.

Sherman \& Co. n.

Stallard, L. $n$

Troll \& Co. $n$

Walker, George $f$.

Watts \& Davis $n$.
Louisville, Jefferson co

Louisville, Jefferson co 585 Fourth Ave., Louisville

St. Matthews, Jefferson co

Louisville, Jefferson co

Louisville, Jefferson co

Newport, Campbell co

Louisville, Jefferson co

Milton, Trimble co

Clinton, Hickman co

I 325 East Broadway, Louisville

Louisville, Jefferson co

Louisville, Jefferson co

Woodburn, Warren co

Louisville, Jefferson co

Louisville, Jefferson co

Louisville, Jefferson co

\section{LOUISIANA.}

Auger, Bernard A. $f$

Baker, Edward $f$.

Barinque, Philip $f$

Bechler, Louis $f$.

Bogle, A. V. n

Bremer, Joseph, Jr. $f$

Buel, Peter $n$

Clingman, A. K. $n$

David, M. $f$.

Despomier, H. A.

Dolder, John $f$

Dubo, John $f$

Eblen, John $f$

Etienne, Oliard $f$.

Fonta, Jules $f$
- Lepage near Seventh Street, New Orleans

St. Andrew near Chestnut Street, New Orleans

N. W. cor. Peters Ave. and Camp St., and 688 Magazine St., New Orleans

Taylor between Prytania and Pitt Sts., New Orleans

Shreveport, Caddo co

N. E. cor. Eighth and Coliseum Sts., New Orleans

New Orleans

Homer, Claiborne co

5or Baronne St., New Orleans

470 Carondelet St., New Orleans

N. E. cor Pleasant and Chestnut Sts., New Orleans

412 St. Andrew St., New Orleans

East Side Hurst between Arabella and Nashville Sts., New Orleans

N. E. cor. Washington Ave. and Carondelet St., New Orleans

Hurst between State and Webster Sts., New Orleans 
Frotscher, Richard n

Gaerdner, Mrs. Catherine $f$.

Hablutzel, George

Howarth, James $f$

Jaeger, Louis $f$

Lacy, T. J. n

Laurent, John B. $f$

Leber, Jules $f$

Lemaire, J. B. $f$.

Lewis, T. C., \& Co. $n$

Little, Robert N. $f$

Mahar, John $f$.

Maitre \& Cook $f \cdot$.

Maitre, Reinhardt $f$

Menard, John H. $f$

Miller, Joseph $f$.

Morris, Thomas, \& Co. n

Muller, Joseph $f$.

Nelson, John

Normand, J. L. $n$.

Pons, Emile $f$.

Rapp, Joachim $n$.

Schultz, Conrad $f$.

Schuler, F. $f$.

Schwarz, Louis $f$.

Sibley, B. C. $n$

Simons, R. \& C. $f$

Singer, Mrs. Caroline $f$

Stoner, G. IV. $n$

Thierry, Mrs. Jane $f$

Thomas, Christian $f$.

Valentine, Valentine $f$.
New Orleans

Dauphin between Lesseps and Poland Sts., New Orleans

S. E. cor. Chestnut and Pleasant Sts., New Orleans

Dryades bet. Dufossat and Soneat Sts., New Orleans

Joseph bet. Laurel and Constance Sts., New Orleans

Washington, St. Landry co

325 Marais St., New Orleans

S. W. cor. Washington and Carondelet Sts., New Orleans

St. John bet. White and Dupre Sts., New Orleans

Shreveport, Caddo co

S. W. cor. Hurst and State Sts., New Orleans

Cor. Adams and St. Charles Sts., New Orleans

976 Magazine St., New Orleans

New Orleans

Carondelet bet. Delord and Calliope Sts., New Orleans

Esplanade, N. W. cor. Grande Route St. John, New Orleans

Shreveport, Caddo co

N. W. cor. Canal and Dauphine Sts., New Orleans

Metairie Road bet. Canal and New Basin, New Orleans

Marksville, Avoyelles co

I 58 Kerlerec St, New Orleans

I 2 St. Philip St., New Orleans

N. W. cor. Carondelet and Felicity Sts., New Orleans

New Orleans

New Levee bet. Joseph and Arabella Sts., New Orleans

Robeline, Natchitoches co

Igo Camp St., New Orleans

St. Charles near Clinton Sts., New Orleans

Shreveport, Caddo co

Derbigny bet. Hospital and Bayou Road, New Orleans

N. E. cor. Eighth and Magazine Sts., New Orleans

240 Sixth St., New Orleans 
Vincent, John $f$

Virgin, Edward F. $n$. Wicks, J. $f$.

Wild, Caspar $f$
N. E. cor. Baronne and Washington Ave., New Orleans

New Orleans

Bordeaux, N. W. cor. Coliseum St., New Orleans

I686 Tchoupitoulas St., New Orleans

\section{MAINE.}

Allen, Thomas $f$

Almond \& Pike $f$.

Atwood, Joseph C. $n$

Blanchard, George, \& Bros. $n$

Burbank, E. W. $n$

Burr, John $n$.

Burrows, G. W. $f$.

Cannon, S. T., \& Co. $n$

Casey, Francis $f$.

Chapman, George H. n

Chapman, L. B., \& Co. n

Chase, M. V. B. n

Derwanger, Albert

Derwanger, Joseph A. $f$

Freeman, N. S. $f$.

Gerrish, O. K. $n$.

Goddard, C. IV. $f$.

Gurney, H., \& Co. $f$

Hammond, J. H., \& Son

Ham, O. S. \& E. P. $n$

Hill, O. A. $n$.

Jackson, Thomas $n$

Jerrard, G. IV. P. n

Kane, Thomas $f$.

Kendall \& Whitney $n$.

Kimball, W. R. $f$.

Knowlton, D. H. $n$

Morton, William $f$

Morton, W. E., \& Co. $f$

Moses, F. H. $f$

Peters, R. B. $n$

Rogers, John $f$

Sawyer, W. C. $n$

Sturdevant, Oscar R. $f$.

Sturgis, S. J. $n$

Trufant, Zina $f$

Vickery, James $f$.

Walker, John C. $n$
Bangor, Penobscot co

Calais, Washington co

Winterport, Waldo co

Portland, Cumberland co

Fryeburgh, Oxford co

Freeport, Cumberland co

Fryeburgh, Oxford co

Augusta, Kennebeć co

Bangor, Penobscot co

Portland, Cumberland co

Stroudwater, Cumberland co

Augusta, Kennebec co

Portland, Cumberland co

Portland, Cumberland co

Bridgton, Cumberland co

Portland, Cumberland co

Lisbon Falls, Androscoggin co

Auburn, Androscoggin co

North Berwick, York co

Lewiston, Androscoggin co

Portland, Cumberland co

Stroudwater, Cumberland co

Caribou, Aroostook co

Portland, Cumberland co

Portland, Cumberland co

Bath, Sagadahoc co

Farmington, Franklin co

Portland, Cumberland co

Portland, Cumberland co

Bucksport, Hancock co

Blue Hill, Hancock co

Kittery, York co

Portland, Cumberland co

Portland, Cumberland co

Greene, Androscoggin co

Bath, Sagadahoc co

Portland, Cumberland co

Mechanic Falls, Androscoggin co 


\section{MARYLAND.}

Anderson, Charles H. n

Arning Bros. $f$.

Bagley, Edward A. $n$.

Bain, Miss M. E. $f$

Balderson, George $n$

Baltimore Nurseries, F. Davis \& Co.

Proprietors $n$

Barnard, Norris $n$

Barnhard, John S. $n$

Bassler, Andrew $f$

Bauer, Henry $f$.

Belvidere Nursery, Andrew

Black, Proprietor $f$

Berger, Gustave $f$.

Blair, Alexander $f$

Bolgiano, J., \& Son $f$.

Booth, Joseph, \& Son n

Brackenridge, Archibald

Brackenridge, W. D. n

Bramble, John H. $n$

Brendel, Henry $f$.

Brendel, William $f$

Brunner, Rudolph $f$

Bucher, Charles T. $f$

Burger, Frederick G. $f$.

Burton, William T. $f$.

Canoles, C. E. $f$.

Carpenter, Lewis $f$

Cook, Anthony $f$.

Cook, Charles $f$.

Cook, John $f$.

Cook, William $f$.

Corse, William \& Son $n$

Corse, William \& Sons $n$

Cottingham \& Bro. $n$.

Cromwell, Richard $f$.

Cromwell, Richard $f$.

Cromwell \& Congdon $n$

Davis, F., \& Co. $f$.

Day, Hoff \& Co. $f$

Diedman, B. $f$

L.
Baltimore

38 Penna. Ave., Baltimore

Reckord, Baltimore co

Madison Ave., Baltimore

Colora, Cecil co

- Baltimore and Paca Sts., Baltimore

Still Pond, Kent co

- Denton, Caroline co

28 N. Green St., Baltimore

Belair and North Aves., Baltimore

Eager and the Falls, Baltimore

Baltimore

Monument and Loury Sts., Baltimore 28 S. Calvert St., Baltimore

Elkton, Cecil co

Govanstown, Baltimore co

Govanstown, Baltimore co

East New Market, Dorchester co

4 I S. Burke St., Baltimore

Third Ave. and Dillon St., Baltimore

Homestead, Baltimore City

Friendship, York Road, Baltimore

208 Green Mountain Ave., Bdtimore

Peddicord Lane near Millington Ave.,

Baltimore

Hereford, Baltimore co

7 I Chew St., Baltimore

Mulbury and Arlington Aves., Baltimore

Mulbury and Arlington Aves., Baltimore

74 N. Charles St., Baltimore

72 N. Arlington Ave., Baltimore

Belair Ave., P. O. Box 248, Baltimore

Orangeville, Baltimore co

62 Light St., Baltimore

5 I Light St., Baltimore

Brooklyn, Anne Arundel co

Baltimore

Baltimore

Hagerstown, Washington co

I 26 Orleans St., Baltimore 
Eichelberger, H. $f$. Fairley, Thomas $f$ Feast, John $f$

Feast, Samuel, \& Son $f$ Fox, Charles A. $f$. Fraser, William $f$.

Frederick, Henry Galloway, James $f$ Ganges, Joseph A. $f$ Gerben, Charles A. $f$. Gettier, George T. Gifford, Margaret $f$ Gosnell, Mrs. Clara E. $f$ Grant, D.S. $f$ Griffith \& Turner $n$ Gummel, Christian G. $f$ Hajda, John

Halliday, Robert $f$

Halliday, Robert J. $f$.

Hallock, E. D. $f$. Hamilton, Charles $f$ Harold, Thomas G. $f$ Harstke, Morris A. $f$. Held, Charles $f$. Hess, Conrad $f$. Hoffman, C. Calvert $f$. Hoffman, Ephraim $f$. Hohn, Edward A. $f$. Holzborn, Adolph O. n Hornick, Peter A. Jarrell, C. E. $n$. Johnson, Patrick $f$ Kemp, Oliver $f$. Kerr, Jonathan W. $n$. Kirkley, Edward $f$ Kress, E. \& N. $f$. Le Brun, Henry $f$. Le Brun, Joseph $f$ Lee, John $f$.

Lee, IVilliam $f$ Lockley, John Martin, Joseph $f$ Martindale, Amos $n$. Mason, Thomas $f$.
I 88 Madison Ave., Baltimore 235 Druid Hill Ave., Baltimore 271 German St., Baltimore 56 N. Charles St., Baltimore I 36 Dolphin St., Baltimore 277 E. Baltimore St. and Belair Ave. opposite Schuetzen Park, Baltimore Iog S. Ann St., Baltimore Waverley, Baltimore City I08 IV. Preston St., Baltimore Barclay near Sixth Sts., Baltimore 102 W. Townsend St., Baltimore I88 E. Fayette St., Baltimore " Baltimore

Govanstown, Baltimore co Baltimore Baltimore

North Ave. near Baltimore Cemetery, Baitimore

Liberty Road near City Limits, Baltimore

$238 \mathrm{IV}$. Baltimore St. and N. E. cor. Penna. Ave. and Dolphin St., Baltimore

Balderston and Charles St., Baltimore Waverly, Baltimore co Kingston, Somerset co Lanvale near Charles Sts., Baltimore 24 S. Eutaw St., Baltimore IVaverly, Baltimore co Madison and North Aves., Baltimore Baltimore 267 N. Stricker St., Baltimore Frostburg, Allegheny co 53 Division Ave., Baltimore Hillsboro, Caroline co Baltimore

Lauraville, Baltimore co Denton, Caroline co 320 Fort Ave., Baltimore North St. near Belair Ave., Baltimore 65 E. Baltimore St., Baltimore Io Bank St., Baltimore Quaker Lane near York Road, Baltimore

422 E. Monument St., Baltimore Elkton, Cecil co 335 Eastern Ave., Baltimore Zion, Cecil co 193 Myrtle Ave., Baltimore 
McCawley, Patrick $f$ McColgan, Charles $f$. McNeal, Andrew $f$ Millman, F. X. $f$. Moses, James $f$. Muller, John H. Nicholson, R. G. $f$ Patterson, Andrew $f$ Patterson, Gilbert H. Patterson, Robert $f$ Pentland, James $f$ Pentland, Jefferson $f$ Perlich, Herman $f$ Peters, Randolph $n$ Precht, Theodore $f$ Pritchard, John W. $f$ Rieve, Werner C. $f$ Roche, Joseph C. $f$ Rouck, Lewis $f$ Ruppert, F. Louis $f$ Russell \& Geise $n$ Sands, S. $f$ Schewa, John $f$ Schmidt, Robert $f$ Scott, A. $f$.

Simcoe, J. S. Smith, George V. $f$ Stewart, Lemuel Stoll, A. $f$.

Sutton, Thomas V. $f$. Taylor, Colin F. $n$

Thomas, Mrs. M. J. $f$. Tredway, Aquilla E. $f$. Waldenberg, C. $f$. Wiedey, John $f$. Wright, Isaac H., \& Son $n$. Zentgrof, Mrs. Sophia $f$ Zink, Henry $f$.
I 32 Conway St., Baltimore Baltimore 38 Penna. Ave., Baltimore Cumberland, Allegheny co Barclay near Sixth St., Baltimore Waverly, Baltimore co Chestertown, Kent co York Road near Sixth St., Baltimore Waverly, Baltimore co I33 W. Madison Ave., Baltimore 27 N. Charles St., Baltimore 319 Greenmount Ave., Baltimore 183 S. Broadway, Baltimore

Denton, Caroline co Linden Ave., Baltimore 242 German St., Baltimore 42 Warren Ave., Baltimore 12 Wilson St., Baltimore Homestead, Baltimore I 46 West St., Baltimore Baltimore I 28 W. Baltimore St., Baltimore 506 Penna. Ave., Baltimore Baltimore Waverly, Baltimore co North East, Cecil co

Baltimore Baltimore Baltimore Baltimore Galena, Kent co 83 N. Charles St., Baltimore Havre de Grace, Harford co Waverly, Baltimore co 203 Madison Ave., Baltimore East Newmarket, Dorchester co Brooklyn, Anne Arundel co Govanstown, Baltimore co

\section{MASSACHUSETTS.}

Adams, C. T. $n$ Adams, J. W. $n$ Ago, John P. $f$ Allen's Nursery $n$
West Medway, Norfolk co Springfield, Hampden co Warren St., Dorchester, Boston Springfield, Hampden co 
Angevine, Bros. $f$

Arey, Thomas $n$.

Armstrong, G. S. $n$

Assig, A. $f$

Atwill, W. A. $f$

Babbit, C. D. $f$

Bamford, Sidney $f$

Barrett, Thomas $f$

Bassett, George F. n

Beals, E. B. $f$

Becker, Frank, \& Co. $f$

Belcher, A. F. $f$.

Beverley, W. R. $f$

Blanchard, John W. $f$.

Bock, William A. $f$

Borden, Clark P. $f$

Bowditch, William E. $f$

Boyle, Neal $f$

Bragg, B. L., \& Co. $f$.

Breed, Edward W. $f$.

Brewster, C. H. $f$.

Brigham, Charles G. $f$.

Broughton, H. L. $n$

Brown, William S. $f$.

Bryant, George $n$.

Buffum, C. M. $n$.

Buffinton, F. $f$.

Cadwell, C. H. $f$.

Cafrey, A. T. $f$.

Calder, Aug. P. $f$.

Callender \& Knapp f .

Cartwright. G. n .

Carney, Hugh A. $f$

Cavannagh, L. $f$.

Chamberlain, John $f$.

Chandler, G. F. $f$.

Chase, R. G., \& Co. $f$.

Clapp, E. B. $f$.

Clark, E. W., \& Son $f$.

Clark, J. P. $f$

Close, O. $n$

Cole, Seth L. $f$

Coleman, J. L. $f$

Cook, W. W. $f$.

Cooledge, G. M. n

Cox, C. A. $f$.

Cox, Thomas A.

Cressey, George W. $f$.

Crawford, A. W. $f$
Melrose, Middlesex co

Yarmouth Port, Barnstable co

Lawrence, Essex co

Florence, Hampshire co

Lynn, Essex co

Berkley, Bristol co

Lynn, Essex co

Washington, near Green, J. P., Boston

Holyoke, Hampden co

Greenfield, Franklin co

Cambridgeport, Middlesex co

Foxboro, Norfolk co

Rockland, Plymouth co

Park and Commercial Sts., Dorchester, Boston

North Cambridge, Middlesex co

Taunton, Bristol co

649 Warren St., Roxbury, Boston

Clarence Place, Dorchester, Boston

Springfield, Hampden co

Clinton, Worcester co

Northampton, Hampshire co

Hudson, Middlesex co

Marblehead, Essex co

New Bedford, Bristol co

Elmwood, Plymouth co

Salem, Essex co

Fall River, Bristol co

Ashburnham, Worcester co

37 Tremont St., Boston

I 27 Tremont St., Boston

Wellesley Hills, Norfolk co

Dedham, Norfolk co

Somerville, Middlesex co

Amesbury, Essex co

Lynn, Essex co

South Lancaster, Worcester co

8 Pemberton Square, Boston

Near Mt. Vernon, Boston

Springfield, Hampden co

I Park St., Boston

Worcester, Worcester co

Gloucester, Essex co

North Adams, Berkshire co

Worcester, Worcester co

Sharon, Norfolk co

Dorchester, Boston

Olney St. Place, Boston

Salem, Essex co

Medford, Middlesex co 
Croughan, P. $f$.

Cruickshanks, G. n

Cruikshank, J. F. $f$

Cummisky, P. $f$.

Cunningham, S. W. $f$.

Cushing, Charles H. $n$. Cutler, George P. $f$

Cutler, S. M. $n$

Dave, C. R. $f$

Davenport, E. N. $f$

Davenport, Lyman $f$

Davis, Mrs. J. W.

Deady, Arthur $f$.

Dee Bros. $f$.

Delay \& Meade $f$

Dickinson, A., \& Son $n$

Dillon, John H.

Dinsmore, W. J. $f$

Donavan, F., \& Co. $f$.

Doogue, Wm. $f$

Dooley, David $f$.

Downing, Henry H. $f$.

Doyle, Wm. E. $f$.

Draper, James $n$.

Drury, Lyman $n$

Duane, J. $f$.

Earle, Wm. H. n.

Eaton, B. Ellis $f$.

Eaton, H. n.

Eaton, H. N. $n$

Ege, John P., \& Co. $f$.

Esterbrook, James $f$.

Ewell, Wm. S., \& Son $f$

Farquhar, Robert

Farquhar, R. \& J., \& Co. n

Fewkes, E. $f$

Fielding, H. A. $f$.

Fisher, F. C. $f$

Fisher, Sewall $f$

Fletcher, Rufus B. $n$

Flynn, E., \& Co.

Forbes, John G. $f$

Foster, J. A.

Foster, L. H. $f$

Gage, Edwin V. $n$
Arlington Place, Brighton, Boston

Fitchburg, Worcester co

Worcester, Worcester co

Dracut, Middlesex co

Salem St. corner Baste Place, W.

Roxbury, Boston

Wellesley Hills, Norfolk co

Taunton, Bristol co

Holliston, Middlesex co

I689 Washington St., Boston

Mather St., Dorchester, Boston

Readville, Norfolk co

Holyoke, Hampden co

Rear 219 M St., Boston

I04 Tremont St., Boston

Park and Stanford Aves., Dorchester,

Boston

Cambridgeport, Middlesex co

Columbia and Bowdoin Aves., Dorchester, Boston

North Cambridge, Middlesex co

Natick, Middlesex co

849 Washington St., Boston

North Attleboro, Bristol co

Lynn, Essex co

43 Tremont St., Boston

Worcester, Worcester co

Worcester, Worcester co

West Medford, Middlesex co

Worcester, Worcester co

Brockton, Plymouth co

Middlesex, Middlesex co

South Sudbury, Middlesex co

4I I Warren Si., Roxbury, Boston

Swansea, Bristol co

Haward near Bird St., Dorchester,

Boston

40 Temple St., Boston

I9 S. Market St., Boston

Newton Highlands, Middlesex co

Lowell, Middlesex co

South Sudbury, Middlesex co

Framingham, Middlesex co

Ayer, Middlesex co

Lawrence, Essex co

3070 Washington St., Boston

Dorchester Ave., corner Mather St.,

Boston

Kings, Dorchester, Boston

Bradford, Essex co 
Gale, W. F. $f$

Gallagher, M. $n$.

Galvin Bros.

Galvin, John

Gardner Bros. $n$.

Gardner, C. P. $f$.

Geist, Justus $f$.

Gill, E. M. n

Gillett, Edward n

Ginnis, M. \& J. $f$

Goodall, L. W. $n$.

Goodwin, Samuel T. $f$.

Goodwin, Thomas W. $f$

Gordon, Jason $f$.

Gormley, John $f$.

Gormley, Patrick $f$

Graves, G. $f$.

Green, Stephen $f$.

Greenlaw, Alexander $f$

Gregory, James J. H. n

Grimmer, Charles P. $f$

Grout, H. $f$.

Hall, O. L. $f$

Halley, Thomas D. $f$.

Hannan, William $f$.

Hanson, J. F. $n$

Harvey, C. H. \& Co. $f$

Haskell, Edward S. $f$.

Hathaway, A. B. $f$

Hathaway, A. S. n

Hathaway, Wm. A. $f$.

Herrick, Nelson J. $f$.

Hill, George H. $f$

Hill, J. Henry $n$.

Hilton, Leander $f$

Holbrook, Gennet L. $f$

Holbrow, H. E. $n$

Hooper, Thomas $n$.

Hovey, C. M., \& Co. $f$.

Howland, Edward $f$.

Hoxie, D. E. $f$

Hughson, L. T. $f$.

Husband, A. \& M. A. $f$

Hutchins, Mary H. $f$.

Hutchinson, B. $f$.

Innisfallen Green Houses $f$

Jackson, Joseph $f$

Jennings, George S. $f$.

Jennings, W. H. $f$

Jephson, V. M. n
West Springfield, Hampden co

Marlboro, Middlesex co

6I Tremont St., Boston

Wheatland Ave., Dorchester, Boston

Nantucket, Nantucket co

Newburyport, Essex co

Melrose, Middlesex co

Medford, Middlesex co

Southwick, Hampden co

8 Winter St., Boston

Amherst, Hampshire co

Lynn, Essex co

Newburyport, Essex co

Adams and Dickens St., Boston

I I Tremont St., Boston

Worcester, Worcester co

I 8 Bedford St., Boston

Amesbury, Essex co

2023 Washington Ave., Boston

Marblehead, Essex co

5 I West St., Boston

Springfield. Hampden co

Pittsfield, Berkshire

Lawrence, Essex co

Park St., Dorchester, Boston

Winchester, Middlesex co

69 Tremont St., Dorchester, Boston

New Bedford, Bristol co

New Bedford, Bristol co

Oxford, Worcester co

New Bedford, Bristol co

306 Main St., Springfield, Hampden co

Haverhill, Essex co

Amesbury, Essex co

Ware, Hampshire co

Boston, Suffolk co

Dorchester, Boston

Bridgewater, Plymouth co

I6 S. Market St., Boston

East Hampton, Hampshire co

Northampton, Hampshire co

Milton, Norfolk co

Taunton, Bristol co

Cambridge, Middlesex co

I93 Tremont St., Boston

Pittsfield, Berkshire co

Fitchburg, Worcester co

West Brookfield, Worcester co

Newton, Middlesex co

Worcester, Worcester co 
Johnson, J. J. $f$.

Kaulbach, Charles $\mathrm{H}$. $f$

Keene, Cyrus R. n

Kendall, William P. $f$.

Keniston, J. A. n .

Keyes, Charles A. $f$.

Kinney, C. M. $n$.

Kinney, F. J. $n$.

Lackey, Andrew $n$

Lang, H. F. A. $f$.

Larcom, Benjamin $f$.

Lavler, John $f$.

Leonard, George E. $f$.

Lewis, John A. $n$.

Lindsay, F. G. $f$.

Little, Samuel $f$.

Lord, Andrew $f$.

Lothrop, H. A. $f$.

Love, William $f$.

Low, Aaron $n$

Luce, William T. $f$

Lyman, E. M. $n$.

Lyons, Thomas F. $f$

Mack, John $f$

Macomber, J. H. $f$

Makepiece, A. D. $n$

Mandall, Jonathan A. $f$

Mandell, J. W. $f$.

Mann, L. W. $n$.

Mann, H. W. $f$.

Manning, J. W. n

Mansfield, Henry A. $f$

Marfield \& Putnam $f$.

Marroth, John $f$.

Mason \& Co. $n$.

Mathison \& Bunker $f$.

Merrill, William $f$

Miller, Andrew $f$.

Minton Bros. $f$

Moony, John $f$

Morrey, E. T. $f$

Morse, E. P. $f$

Mulhall, W. B. $f$

McCarty, Timothy $f$

McDonald, J. C. $f$

McDowell, T. $f$.

McGee \& Geary $f$

McGrath, John \& Son $f$
Newton, Middlesex co

Haverhill, Essex co

Cohasset, Norfolk co

Boston, Suffolk co

Newburyport, Essex co

Worcester, Worcester co

Northampton, Hampshire co

Worcester, Worcester co

Haverhill, Essex co

Worcester, Worcester co

Beverly, Essex co

Milton St., corner Granite Ave., Dorchester, Boston

Foxboro, Norfolk co

Dighton, Bristol co

Taunton, Bristol co

Warren St., Dorchester, Boston

Lynn, Essex co

Sharon, Norfolk co

Withington St., Dorchester, Boston

Essex, Essex co

New Bedford, Bristol co

Springîeld, Hampden co

Canton, Norfolk co

Beverly, Essex co

Fall River, Briston

Hyannis, Barnstable co

Somerville, Middlesex co

I 38 Main St., Boston

Randolph, Norfolk co

Stoughton, Norfolk co

Reading, Middlesex co

Newtonville, Middlesex ro

Danvers, Essex co

5 Hudson St., Dorchester, Boston

I I Central St., Boston

147 B. Tremont St., Boston

West Newbury, Essex co

I 20 Tremont St., Boston

Corner Adams and Shelton Sts., Dorchester, Boston

River St., near Cedar Lane, Boston

Boston

Dedham, Norfolk co

Ioo Tremont St., Boston

West Newton, Middlesex co

$20 \frac{1}{2}$ Temple Place, Boston

Emerson, near K St., Boston

Salem, Essex co

Cottage City, Dukes co 
McLaren, A. M. $f$

McLaughlin, Charles $f$ McNulty, E. P. $f$.

Nash, George $f$.

Neil, Samuel $f$.

Nevins, J. K. $n$.

Newman, J, \& Son $f$.

Newman, Miss M. S. $f$

Nichols, Samuel $f$

Norton Bros. $f$

Nugent, James $f$.

Ober, Charles $f$

Oldrieve, R. $f$

Oliver, John $f$

O'Brien, James $f$.

O'Brien, P., \& Son

Page, W. E. $f$.

Parker, J. L. $f$

Parker, R. G., \& Co. $f$

Parker \& Wood $n$

Parker, Mr.

Parrott, Geo. O. $f$.

Pasell, F. H.

Patten \& Co.

Patten, George W. $f$

Payne, R. $f$.

Pearce \& Esterbrook $f$

Phelps, C. H. $f$.

Phillips, Alfred $f$.

Pierce, William $f$.

Pierce, W. G. $f$.

Power, Charles J. $f$

Pratt, C. S. $n$

Pulsifer, George H. $f$.

Punchard, John H. $f$.

Putnam, Henry W. $f$.

Rawson, W. W., \& Co. $n$

Reddy, Nicholas

Reed, A. A. $f$

Regan, Frank A.

Richardson Bros.

Robbins, E. R., \& Co. $f$

Robbins, H. H. $n$

Roberts Bros. $f$.
Corner Washington and Morton Sts.,

\section{Boston}

Haverhill, Essex co

Saven Hill Ave., Boston

Cambridgeport, Middlesex co

Washington, opposite Park, Dorchester, Boston

Montague, Franklin co

7 Tremont St., Boston

27 A. Beacon, Boston

Cohasset, Norfolk co.

Corner Bowdoin and Olney Sts., Dorchesrer, Boston

Lamartine, near Cedar Ave., J. P., Boston

Beverly, Essex co

Newton, Middlesex co

Lowell, Middlesex co

Main St., Harvard Square, Boston

Cambridge, Middlesex co

St. Mary's, near Brighton Ave., Boston

Rock Bottom, Middlesex co

Corner Washington and Rockwell

Sts., Dorchester, Boston

49 N. Market St., Boston

Stoneham, Middlesex co

Lynn, Essex co

New Bedford, Bristol co

Lowell, Middlesex co

Lowell, Middlesex co

Newtonville, Middlesex co

Fall River, Bristol co

South. Framingham, Middlesex co

Marshfield, Plymouth co

New Bedford, Bristol co

Swansea, Bristol co

South Framingham, Middlesex co

North Reading, Middlesex co

Gloucester, Essex co

Salem, Essex co

Salem, Essex co

43 S. Market St. , Boston

Fall River, Bristol co

South Abington, Plymouth co

Lawrence, Essex co

Melrose, Middlesex co

South Abington, Plymouth co

South Abington, Plymouth co

Hyde Park, Norfolk co 
Robinson, J. C. $n$

Rogers, Samuel C. B. $f$

Ross, S. W. $f$

Rough, James $f$.

Ruggles \& Minott

Ruggles, Otis A. $f$

Salisbury, Stephen

Saunders, S. O. $n$

Sawyer, Sylvanus

Seaverns, George $f$

Severy, L. F.

Shaw, E. D.

Sheehan, Jeremiah $f$.

Sheehan, John $f$.

Sheppard, E., \& Sons $f$

Showers, Patrick $f$

Small, N. S. $f$

Smith, C. H. n

Smith, David $f$

Smith, G. N.

Smith, H. E.

Smith, H. N.

Smith, Mark

Snow, T. M. $f$

Spooner, William H. $f$

Steiert Bros. $f$

Stewart, William J. $f$.

Story, E. A. $f$

Strong, William C. \& Co. $n$

Stroud, John E. $f$.

Sweet, William O. $n$

Tailby, Joseph $f$.

Talbot, W. Allen $f$

Tapper, Thomas $f$

Tasker, G. H. $n$

Taylor, A. $f$.

Taylor, D. L. $f$

Teel, James M. $f$.

Temple, F. L. $u$.

Thayer, Charles D. $f$.

Thompson. C. H. n

Thornton Bros. $f$.

Townsend, Hammer \& Co. $f$

Twombley, S. W., \& Sons $f$

Vass, William J.

Vaughn, J. A., \& Co. n Walker \& Co. $n$

Walkup, E. A. $f$
Boston Highlands, Boston

9 Tremont Place, Boston

Chestnut Hill Ave., Brighton, Boston

I 559 Washington St., Boston

Three Rivers, Hampden co

I 58 Tremont St., Boston

Worcester, Worcester co

Everett, Middlesex co

Fitchburg, Worcester co

Auburndale (P. O. Box 63), Boston

5 I6 Tremont St., Boston

Holyoke, Hampden co

Springfield, Hampden co

Lynn, Essex co

Lowell, Middlesex co

98 Old Harbor, Boston

Foxboro, Norfolk co

Holyoke, Hampden co

Fall River, Bristol co

Newton, Middlessx co

Wales Place, Dorchester, Boston

South Sudbury, Middlesex co

Fall River, Bristol co

Old Colony Depot, Boston

Centre St., near Boylston, J. P. Boston

3039 Washington St., Boston

67 Bromfield St., Boston

N. Beacon, Brighton, Boston

Nonantum Hill, Brighton, Boston

West Newton, Middlesex co

West Mansfield, Bristol co

Wellesley, Norfolk co

Norwood, Norfolk co

Canton, Norfolk co

Attleboro, Bristol co

Io Meriden East, Boston

Melrose, Middlesex co

Lynn, Essex co

Somerville, Middlesex co

Worcester, Worcester co

Boston

Lawrence, Essex co

Hyde Park, Norfolk co

I6I Tremont St., Boston

Corner Cyprus and Walnut Sts.,

Brookline, Boston

Carver, Plymouth co

Dennis and Dudley Sts., Boston

Framingham, Middlesex co 
Warren, H. F. $f$.

Watson, B. M. $n$.

Watts, H. B. n

Webb, William $f$

Welch Bros. $f$

Weld, C. E. $f$

Wells, Benjamin T. $f$.

Welton, Charles

Wentworth, William G. $f$

Westgate, S. F. $n$

Whipple, L. K. n .

White \& Co. $f$.

White, W. B. $n$.

Wilde, Mrs. C. B. $f$

Wilkinson, John, \& Son $f$.

Wilson, George W. $f$.

Winter Bros.

Wood, Edmund M., \& Co. $f$

Wood, Elijah W. $f$

Woodman, E. \& C. $f$.

Woolson, E. L. $f$

Worch, Charles $f$.

Wright, Alida $n$.

Young, Henry $f$.

Young, Thomas $f$

Zingierbel, D. $f$.
Waltham, Middlesex co

Plymouth, Plymouth co

Leicester, Worcester co

Quincy, Norfolk co

I65 Tremont St., Boston

Roslindale, Boston

I8 Hawley St., Boston

Corner Washington and Morton Sts.,

Forest Hill Station, Boston

Winchester, Middlesex co

Long Plain, Bristol co

- Newburyport, Essex co

Pittsfield, Berkshire

Norwood, Norfolk co

I63 Tremont Place, Boston

Springfield, Hampden co

Malden, Boston

Mansfield, Bristol

- 34 Oliver St., Boston, Suffolk, and

Natick, Middlesex co

West Newton, Middlesex

Danvers, Essex co

Hopkinton, Middlesex

307 Warren St., Roxboro, Boston

Cambridge, Middlesex co

Lynn, Essex co

Somerville, Middlesex co

Needham, Norfolk co

\section{MICHIGAN.}

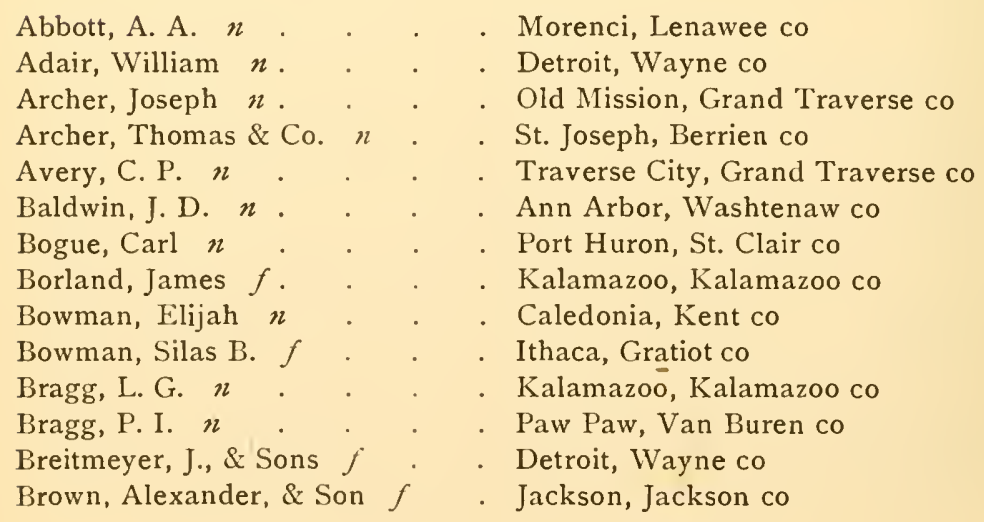


Bullard, S. F. $n$. Childs, Lewis E. $f$ Conkwright, A. A. n

Consaul, Lewis $f$. Cousins \& Hall $f$. Crawford, J. T. $f$. Davis, J. N. $n$. Dew, Henry F. $n$. Dumphrey, W. C. $n$ Edgell, H. J. $n$. Edmiston, D. G. $n$ Efferts, Andrew $f$ Escher, J. F. $n$ Farnum, Mrs. H. T. $f$. Ferry, D. M., \& Co. $n$. Ford, John, \& Sons $f$. Fulkerson, W. G. $n$. Galvin \& Jones $f$. Ganshorn, Jacob $n$ Gœtter, G. R. $f$. Goldie, William $f$

Greening, J. C. W., \& Sons $n$ Hallett \& Donaghy $n$. Hasselbring, B $f$. Haven, Edw. M. n Hays, A. E. $f$

Hendricks, Frederick $n$ Hopkins, J. L. $n$. Hoskins, Thomas $n$ Hovey, J. A. $f$. Hudson, W. O. $n$. Hughes, W. $n$

Ilgenbritz, J. E., \& Sons $n$. Irvine, John Jeffrey, J. $n$. Johnson, W. W. $n$ Jones, A. B. n

Kaichen, Pauline C., \& Co. $f$ Kellogg, R. M. $n$ Knowlton, C. C. $n$ Kribbs, J. S. $f$. LaFleur, G. H. $n$. Lanning, Joseph $n$ Loud, George B. n LeValley, E. $n$. Lewis, S. B. n Lucia, Frederick $n$ Lyon, T. T. $n$ Mackintosh, A. $f$. Mann, R., \& Son $f$
Coldwater, Branch co Ypsilanti, Washtenaw co Chelsea, Washtenaw co Adrian, Lenawee co Ann Arbor, Washtenaw co Pontiac, Oakland co Marshall, Calhoun co St. Johns, Clinton co Battle Creek, Calhoun co South Haven, Van Buren co Adrian, Lenawee co Pontiac, Oakland co Albion, Calhoun co Hillsdale, Hillsdale co Detroit, Wayne co Detroit, Wayne co Ovid, Clinton co Detroit, Wayne co Ann Arbor, Washtenaw co Ionia, Ionia co Niles, Berrien co Monroe, Monroe co Hillsdale, Hillsdale co Flint, Genesee co Bloomingdale, Van Buren co Advance, Charlevoix co Zeeland, Ottawa co South Haven, Van Buren co Waucedah, Menominee co Grand Rapids, Kent co Allegan, Allegan co Hillsdale, Hillsdale co Monroe, Monroe co Bay City, Bay co Kalamazoo, Kalamazoo co Snowflake, Antrim co Lawton, Van Buren co Detroit, Wayne co Ionia, Ionia co. Old Mission, Grand Traverse co Sand Beach, Huron co Allegan, Allegan co South Haven, Van Buren co Romeo, Macomb co Ionia, Ionia co Monroe, Monroe co Flushing, Genesee co South Haven, Van Buren co Detroit, Wayne co Lansing, Ingham co 
Merrick, C. J. $n$. Milliken, R. n Mylins, A. D. $f$. McCrea, J. F. $n$ McLeod, Archibald $f$. McManus, Peter n Nagle, F. D. $n$. Nye, Silas $n$.

Palmer, C. E. $n$ Parker, F. H. $n$. Penfield, W. S. $n$. Phillips \& Williams $n$ Potter, E. M. $n$. Randall, Hiram n Randall, J. M. $n$. Renwick, R. $n$. Reynolds, E. H. $n$ Roby, E. A. n Rœthke, William $f$ Rowley, S. G. $n$. Rundell, Truman $f$ Schrœter, Bruno $f$

Schultz, C. H $f$

Severace, S. M. n Smith, C. F. $f$

Smith, Mrs. N. M. $f$

Smith, N. E. $\quad x$

Smith, W. T. $n$ Staples, Delos $n$. Stearns, J. N. $n$ Stowell, Charles B. $f$. Strelmger, Julian $f$ Taber, W. B. \& M. $f$. Taplin, Stephen Taylor, George $n$ Thomas, H. F. $n$ Van Hafften, C., \& Sons $f$. Vanstambrook, E. $n$. Vernor, James $f$. Voorhees, W. G. n Wakefield, F. A. n Waltz, Fred. $n$

Warner, F. A. $f$. Watson, R. H., \& Son $f$ Wetterling, M. $n$. Wolff, Charles $f$. Woodford, Harry E. $f$. Wright, F. L. $n$ Zinnen, John $f$
Adrian, Lenawee co Almont, Lapier co Detroit, Wayne co Detroit, Wayne co Romeo, Macomb co Howell, Livingston co South Haven, Van Buren co Romeo, Macomb co South Haven, Van Buren co Eaton Rapids, Eaton co Detroit, Wayne co South Haven, Van Buren co Kalamazoo, Kalamazoo co Martinsville, Wayne co Adrian, Lenawee co Grand Rapids, Kent co Monroe, Monroe co Sparta Centre, Kent co Saginaw, Saginaw co Ypsilanti, Washtenaw co

Bay City, Bay co

Elmwood Ave. and Lafayette St., Detroit

New Buffalo, Berrien co

West Sebewa, Ionia co

Adrian, Lenawee co

Sturgis, St. Joseph co

Ionia, Ionia co

Schoolcroft, Kalamazoo co

West Sebewa, Ionia co

Kalamazoo, Kalamazoo co

Hudson, Lenawee co

I 3 Bates St., Detroit, Wayne co

Detroit, Wayne co

Detroit, Wayne co

Kalamazoo, Kalamazoo co

Jackson, Jackson co

Kalamazoo, Kalamazoo co

New Baltimore, Macomb co

Detroit, Vayne co

South Frankfort, Benzie co

South Haven, Van Buren co

Detroit, Wayne co

Saginaw, Saginaw co

Lapeer, Lapeer co

Ionia, Ionia co

Detroit, Wayne co

Battle Creek, Calhoun co

Plainfield, Livingston co

Detroit, Wayne co 


\section{MINNESOTA.}

Andrews, J. P. $u$.

Baldwin, S. D. L. $n$

Brimhall, W. E. $n$

Bunde, A. H. $f$

Cook, Martin W. $n$

Fleischer, John $f$.

Frank, Carl $n$

Gill, E. A. $f$.

Gilmore, J. $n$

Hill, H. B. $n$

Kilbourne, F. M.

Kilvington, W. C. $f$

Latham, A. W. $n$.

Lempke Bros. $f$.

Lempke, E. F. $f$

Mathews, A. C. $n$

May, L. L., \& Co. "

Mendenhall, R. J. $f$

Metcalf, T. W. $n$.

McComber, Warren $f$

McHenry, William $n$.

Northrup, Breslau \& Co.

Roe, A. D. $f$

Schuman \& Co. $f$

Stevens, Mrs. B. C. $f$

Stubbs, N. J.

Taylor \& Morgan $f$

Vencke \& Haupt $n$

Walton, W. L. $f$.

Wunder, John $f$.
Faribault, Rice co

Cedar Mills, Meeker co

St. Paul, Ramsey co

St. Paul, Ramsey co

Rochester, Olmstead co

St. Paul, Ramsey co

Winona, Winona co

St. Paul, Ramsey co

Richfield, Hennepin co

Duluth, St. Louis co

Lakeville, Dakota co

Minneapolis, Hennepin co

Excelsior, Hennepin co

St. Paul, Ramsey co

St. Paul, Ramsey co.

Lake Benton, Lincoln co

St. Paul, Ramsey co

Minneapolis. Hennepin co

St. Paul, Ramsey co

Duluth, St. Louis co

St. Charles, Winona co

St. Paul, Ramsey co

Stillwater, Washington co

Minneapolis, Hennepin co

Red Wing, Goodhue co

Long Lake, Hennepin co

Forestville, Fillmore co

St. Paul, Ramsey co

Wabasha, Wabaslia co

Winona, Winona co

\section{MISSISSIPPI.}

Brown, R. S. $n$

Coleman, J. J. $f$

Dille \& Son $n$

Fry, D. S. $f$.

Gaines, Coles \& Co. $n$.

Gregory, T. S. $n$

McArthur, Son \& Co. n

Vick, C. W. $f$

Ward, T. G. $x$
Brandon, Rankin co

Columbus, Lownds co

Starkville, Oktibbeha co

Water Valley, Yalobusha co

State Line, Greene co

Vicksburg, Warren co

Meridian, Lauderdale co

Vicksburg, Warren co

Jackson, Hinds co 


\section{MISSOURI.}

Ambrose, A. n

Armstrong, Luther $f$

Arnd, W. P. $f$.

Bailey, Robert E. $n$

Bayles, J. A.

Bayles, Samuel M. $f$

Bell, William M. n

Berdan, A. $f$

Beyer, Charles $f$.

Blair \& Kaufman $n$

Bledsoe, William $n$

Brittain, Robert $n$

Burrows, E. $n$

Bush \& Meissner $n$

Bushnell \& Co. $n$

Bushnell, M. J. n

Butterfield, M. $n$.

Cannon, Charles $f$

Carver, James $n$.

Cassinelli, Angeline $f$.

Chamberlain, F. B., \& Co. $n$

Colman \& Son $n$.

Cox, Samuel N. $f$

Determan, Ernest $n$

Durkas, A. n

Elliard, Charles N. $f$.

Ellison, William $f$

English, C. H. $n$ :

Evans, Edward $f$

Fetter, G. W., \& Bro. $n$

Fillmore, Francis J. $f$.

Flower, Jacob H. $f$

Frow, Richard $f$.

Gaunt, T. W. $n$.

Glahn, August $f$.

Goodman, L. A. $f$

Griffith, N. S. $n$.

Guth, Theobald $f$

Gutzman, Frank $f$
Nevada, Vernon co

2950 Easton Ave., St. Louis

I 5 South 7 th St., St. Louis

Fulton, Callaway co

Lee's Summit, Jackson co

Michigan Ave. cor. Ellwood, St.

Louis

Miami, Saline co

Grand, near St. Louis Aves., St. Louis

S. W. cor. Grand Ave. and Gravois,

St. Louis

Kansas City, Jackson co

Pleasant Hill, Cass co

Half Rock, Mercer co

Canton, Lewis co

Bushberg, Jefferson co

I7 South Main St., St. Louis

St. Louis

Lee's Summit, Jackson co

2740 Olive St., St. Louis

Guilford, Nodaway co

3 I I South 4th St., St. Louis

I9 Main St., St. Louis

St. Louis

St. Joseph, Buchanan co

St. Louis

Weston, Platte co

605 Washington Ave., St. Louis

2602 Olive St., St. Louis

Sullivan, Franklin co 2org Spring Ave., St. Louis

Strafford, Greene co

S. E. cor. Virginia Ave. and Oceola, St. Louis

Cor. Aubert and Knight, St. Louis

Natural Bridge, near Clay Ave., St. Louis

Maryville, Nodaway co

Boonville, Cooper co

Westport, Jackson co

Independence, Jackson co

29 I9 Nebraska Ave., St. Louis

Broadway, opposite Bellefont Cemetery, St. Louis 
Harland, Frank $n$ Hicks, E. H. $f$. Hix, Winfield E. $f$ Holloway \& Skinner $n$ Hugernin, Margaret $f$. Hyman, J. A. $n$. Jeager, Julius E. $f$ Jordan Floral Co. $f$ Juengel, J., \& Son $f$ Juette, H. F. $n$.

Kellogg, George M. $n$. Kelsey \& Co. $n$. Kendall, M. A. $n$. Klockenkemper, Theo, $f$ Knoll, John A. $f$. Knoop, W. W. $f$. Kœnig, Julius $f$.

Kuntz, Charles $n$. Leddle, J. F. $n$

Little, W. S. $n$

Lowen, F. W. $n$.

Luckardt, George P.

Meyer, Andrew $f$

Michel Plant and Seed Co. n

Mitchell, B. F. $n$.

Mohler \& Son $n$.

Murnane, Thomas $f$

Murray, N. F. $n$.

Myers, E. W. $n$

McIntyre, J. W. $f$

Neilson, Hans $f$.

O'Byrne, C. T. $f$.

Palmer, S. C. $f$.

Pape, William $f$.

Patterson, Charles $f$

Paule, A. $f$.

Prenty, C. E. $n$

Pommer \& Lash $n$

Reynolds, Mark L. $n$.

Ringrose, Thomas $n$.

Rivers, C. $n$

Roesch, Herman

Roundtree \& Co., M. J. $n$

Sanders \& Beneke $f$

Schaper, E., \& Co. $n$.

Schnell, H. $n$.

Schray, William $f$

Sharp. Thomas J. n
Canton, Lewis co

Ellardville, St. Louis co

Garfield, near Goode Ave., St. Louis

Butler, Bates co

827 North $4^{\text {th }}$ St., St. Louis

Breckenridge, Caldwell co

I814 Sidney St., St. Louis

706 Olive St., St. Louis

I 833 Linn St., St. Louis

Palmyra, Marion co

Pleasant Hill, Cass co

St. Joseph, Buchanan co

Mt. Vernon, Lawrence co

Sullivan Ave., n 26th St., St. Louis

Virginia Ave., near Delor, St. Louis

Cameron, Clinton co

Florissant Ave., near Marcus Ave.,

St. Louis

Montrose, Henry co

Holden, Johnson co

La Plata, Macon co

Trenton, Grundy co

Oregon, Holt co

3214 South Jefferson Ave., St. Louis

708 Olive St., St. Louis

Butler, Bates co

Cornelia, Johnson co

Cor. Taylor and Evans Ave., St. Louis

Elm Grove P. O., Holt co

Monroe City, Monroe co

Fulton, Callaway co

St. Joseph, Buchanan co

Broadway, near Morin Ave., St. Louis

Kansas City, Jackson co

Pennsylvania Ave., near Utah, St.

Louis

Kirksville, Adair co

St. Charles, St. Charles co

7 Main St., St. Louis

I 5 Main St., St. Louis

Buffalo, Dallas co

Palmyra, Marion co

Moberly, Randolph co

St. Louis

Springfield, Greene co

I 2 I 4 Olive St., St. Louis

St. Louis

Glasgow, Howard co

4IO4 Pennsylvania Ave., St. Louis

Monroe City, Monroe co 
Shults, A. N. $n$

Stuppy \& Reichard $f$.

Stahl, J. M. $f$

Stebbin, IV. S. $n$.

St. Louis Nursery Co. n

Sylvester, E. A. n

Tact, Henry $f$

Taylor, W. A. $f$.

Teas, J. C. $n$

Tholen, C., \& Son $f$

Thomas, John $f$.

Trice Bros. $n$

Viaille, Vincent $f$

Von Canpenhoudt, J. $f$

Waldbert, Alexander $f$

Wallace, M. A.

Wayman, Kennedy \& Co. n Weatherbee, G. W. n .

Weber, Frederick C. $f$

IVild, J. B., \& Bros. $n$.

Willers, Frederick $f$.

Williams, E. $n$

Wilson, James S., \& Bros. $f$

Young, C., \& Son $f$.
Moscow Mills, Lincoln co

St. Joseph, Buchanan co

2 I 7 Olive St., St. Louis

Springfield, Greene co

St. Louis

Osborn, De Kalb co

Taylor, near Easton Ave., St. Louis

Georgetown, Pettis co

Carthage, Jasper co

St. Joseph, Buchanan co

Broadway, near Thatcher Ave., St. Louis

Columbia, Boone co

I I 2 Elliott Ave., St. Louis

- Clagett, near Taylor Ave., St. Louis

3534 Chestnut St. St. Louis

Jackson, Cape Girardeau co

Ravanna, Mercer co

- Utica, Livingston co

1220 Olive St. St. Louis

Sarcoxie, Jasper co

Cor. Capitol Ave. and Winnebago,

St. Louis

Festus, Jefferson co

34 5 Olive St., St. Louis

I 406 Olive St., St. Louis

\section{MONTANA.}

Wells \& Crawford $n$ - Helena, Lewis and Clarke co

\section{NEBRASKA.}

Anderson, A. C. $n$

Arnold, Mrs. Emily E. $f$

Bernard, S. $n$

Carpenter \& Gage $n$.

Carpenter, G. I. $n$

Childs, Edward L. $n$

Clark, G. F. n

Cobbledick, J. $n$.
Creston, Platte co

Omaha, Douglas co

Table Rock, Pawnee co

Fairbury, Jefferson co

Bower, Jefferson co

Crete, Saline co

Odell, Gage co

Republican City, Harlan co 
Craig, Hiram $n$

Cramer, J. W. $n$

Day, R. W., \& Co. $n$.

Donaghue, A. $f$

Eifling, Edward C. $f$.

Evans, John

Festner, F. C. $f$

Forsythe, John $f$.

French, A. n

Furnas, R. W. $n$

Gurney, C. W. "

Harrison, W. A., \& White Bros. $n$

Holmes Bros. $n$

Howe, O. D. $n$.

Kauffman, C. M. n

Long, David $n$

Meyer, Karl

Murray, Peter $f$

Norris, J. B. $n$

Ruthbum, J. C. $n$.

Sawyer \& Co. $f$.

Stephens, E. F. $n$

Stephens, S. L. $n$.

Tannahill, John $n$

Trescher, A. S. $n$

Walcott, H. G. $n$.

Warner, M. M. n

Warren, George F.

Wickham, James

Yonte, John $n$
- Fort Calhoun, Washington co

Hastings, Adams ${ }^{\circ} \mathrm{co}$

- Omaha, Douglas co

- Omaha, Douglas co

- Omaha, Douglas co

- Omaha, Douglas co

Omaha, Douglas co

Fremont, Dodge co

Blair, Washington co

Brownville, Nemaha co

Wakefield, Dixon co

York, York co

Beatrice, Gage co

Table Rock, Pawnee co

Brownville, Nemaha co

Falls City, Richardson co

Fort Calhoun, Washington co

Lincoln, Lancaster co

Fremont, Dodge co

Fairmont, Fillmore co

Lincoln, Lancaster co

Crete, Saline co

Beatrice, Gage co

Columbus, Platte co

Saint Paul, Howard co

Fremont, Dodge co

Fremont, Dodge co

Harvard, Clay co

Salem, Richardson co

Nebraska City, Otoe co

\section{NEVADA.}

Chapin, P. R. $n$. . . Reno, Washoe co

Levy, M. S. $f$. . . . Virginia City, Storey co

\section{NEW HAMPSHIRE.}

Burley, Charles $f . \quad$. . Exeter, Rockingham co

Colby, W. M. $f$. . . . Concord, Merrimack co

Copp, John $n$. . . . Wakefield, Carroll co 
Dodge, Oliver $n$

Dunlap, A. H., \& Son $n$

Ellis Bros. $f$

Folsom, Irwin $n$.

Gordon, Nathaniel $f$.

Hawkins, John $f$.

Hayes, Daniel F. $f$

Hills, W. H. n

Hoitt, C. W. $f$

Holt, IV. P. $n$

Huntress, H. H., \& Son $f$

Hutchinson, M. E., \& Co. $f$

Main, George $n$

Pillsbury, Gorham

Shaw \& Co. $n$

Smith, A. B. $f$

Smith, J. T. $f$

Smith, John, \& Son $f$.

Spangler, W. H. $n$.

Stevens, T. M. $f$.

Stowell, G. H. $n$.

Worthen, F. S. \& Son
Nashua, Hillsboro co

Nashua, Hillsboro co

Keene, Cheshire co

Raymond, Rockingham co

Exeter, Rockingham co

Concord, Merrimack co

Exeter, Rockingham co

Plaistow, Rockingham co

Nashua, Hillsboro co

South Lyndeboro, Hillsboro co

Manchester, Hillsboro co

Portsmouth, Rockingham co

Concord, Merrimack co

Nashua, Hillsboro co

Hill, Merrimack co

Manchester, Hillsboro co

Brentwood, Rockingham co

Brentwood, Rockingham co

Mount Delight, Rockingham co

Littleton, Grafton co

Claremont, Sullivan co

Manchester, Hillsboro co

\section{NEW JERSEY.}

Allen, Edward $n$.

As , S. P. $f$.

Ash, W. H. $f$

Asmus, Ernest $f$.

Atkinson, R. H. $f$

Atkinson, R., \& Son $f$.

Austin, Edward $f$

Baird, David, \& Son $n$

Bauer, Frederick

Baumann, Peter

Baumans, C. E.

Bassett, W. F. $n$.

Batty, J. n

Bedman, Charles

Bedman, William, Sr.

Bedman, William, Jr. $f$

Bedwell, William $f$

Beebe, E. P. $f$

Begerow \& Gerlach $f$.

Binsted, George W. $f$.

Bird, Henry
New Brunswick, Middlesex co South Vineland, Cumberland co South Vineland, Cumberland co

Bergen, Hudson co

Edgewater Park, Burlington co

Beverly, Burlington co

Camden, Camden co

Manalapan, Monmouth co

New Brunswick, Middlesex co

West Hoboken, Hudson co

Rahway, Union co

Hammonton, Atlantic co

Manchester, Ocean co

Rahway, Union co

Woodbridge, Middlesex co

Woodbridge, Middlesex co

Madison, Morris co

Elizabath, Union co

Newark, Essex co

Summit, Union co

Newark, Essex co 
Black, Charles \& Bro. n Blackwell Bros. $n$ Blakeley, J. W. $f$ Blanchard, Henry Bœner, Frederick $f$ Bogie, Alexander $n$ Bohl, F. S. $f$

Bostfeldt, Marianna $n$. Borneman, B. $f$. Bradbury, Joseph $f$ Brandis, Henry F. $n$. Brendt, Ferd. $n$. Brice, J. $f$

Brown, Asa L. $f$

Brown, John $f$

Brown, Leander $f$

Bruns, Herman $n$

Burhaus, J. L. $f$

Burt, Joseph n

Butler, C. $f$.

Butlerton, John $n$

Cain, Nathaniel $n$

Carré, William P. $f$

Carson, William H. $n$.

Carton, John

Chinnick, W. J. $f$.

Chitty, Henry E.

Churchman, John $n$

Cole, R. D. $n$

Coleman, F. F. $f$.

Collins, John S. $f$

Cook, A. T. n

Cook, John A. $f$.

Cook \& Brown $f$.

Cosgrove, Frank $f$

Crowell, H. F. $f$.

Da Costa, J. S. $f$.

Dahl \& Skaarup $f$

Dailey, M. A. $n$

Darseley, George $f$

De Cou, Samuel C. $n$

De Forest, A. V. N. $f$

De Forrest, William H.

Demaray \& Wheeler $f$

Denton, L. J. $n$

Dieffenbach, Charles $n$

Doe, H. \& Son $f$.

Donnely, Thomas
Hightstown, Mercer co

Titusville, Mercer co

Elizabeth, Union co

Newark, Essex co

Cape May, Cape May co

499 Summit Ave., Jersey City

Metuchen, Middlesex co

I 49 Washington St., Hoboken

Passaic, Passaic co

South Orange, Essex co

209 Washington St., Hoboken

47 I Orange St., Newark, Essex co

Madison, Morris co

East Newark, Essex co

Haddonfield, Camden co

Madison, Morris co

Cor. Charles St. and Nelson Ave., Jersey City

Westfield, Union co

Bridgeton, Cumberland co

Summit, Union co

Hammonton, Atlantic co

Harlingen, Somerset co

Mantua, Gloucester co

Crescent and Harrison Aves., Jersey City

Sea Bright, Monmouth co

Trenton, Mercer co

Paterson, Passaic co

Burlington, Burlington co

Bridgeton, Cumberland co

Red Bank, Monmouth co

Moorestown, Burlington co

Clinton, Hunterdon co

Beverly, Burlington co

Madison, Morris co

Madison, Morris co

Atlantic City, Atlantic co

Camden, Camden co

Mt. Holly, Burlington co

Bloomfield, Essex co

Jersey City, Hudson co

Moorestown, Burlington co

Metuchen, Middlesex co

Summit, Union co

Somerville, Somerset co

Plainfield, Union co

I 24 Griffith Ave., Jersey City

Elizabeth, Union co

Madison, Morris co 
Durand, E. W. $n$

Edelefsen, A. T. $f$

Edwards, A. $f$.

Edwards, Stein $f$.

Ellis, C. R. $f$

Ely, E. A. $n$

Emery, P. E. $f$

Engle, Fredericl: $f$

Esler, J. G. \& A. $f$

Felson, Oscar $n$.

Ferris, J. D., \& Co. $n$.

Field, E. T. $n$.

Fithian \& Robinson $n$.

Flumerfelt, M. C. $n$

Force, M. L. $f$.

Fraser, James $f$.

Gamewell Bros. $f$.

Gardner, L. W. $n$

Gibson \& Bennett

Glynn, Thomas $n$

Green, Miss Alice $f$

Greenbrook \& Paterson

Hales, H. W. $f$

Hall, A. G. $f$

Hance \& Borden $n$

Hancock, L. R. $f$.

Harrison, John $f$.

Haviland, J. T. $n$.

Henderson, James $f$

Herr, H. C.

Henry, G. \& L. $f$.

Hewitt, Richard $f$

Hibbins, A. $f$

Hill, W. C $f$

Hoffsteter, John $f$

Holding, John $n$.

Hornecker, Charles $f$.

Horner, C. B. $n$.

Horner, Joel $f$.

Howard, E. U. $f$.

Howard, J. H. $f$.

Hurff, Freeman $n$

Hutchinson, Mrs. J. $n$.

Hunter, A. R. $f$

Hunter, A. R. $f$

Hunter, David $f$.

Jaeger, C. $f$.

Kaiser, Otto $f$.

Knott, Henry $f$.

Knapp, G. R. $n$.
Irvington, Essex co

Bound Brook, Somerset co

Jersey City Heights, Jersey City

Bridgeton, Cumberland co

Avondale, Essex co

Hightstown, Mercer co

Flemington, Hunterdon co

West Hoboken, Hudson co

Saddle River, Bergen co

Merchantville, Camden co

P. O. Box 18, Belleville, Essex co

Red Bank, Monmouth co

Bridgeton. Cumberland co

Danville, Warren co

Morristown, Morris co

Summit, Union co

Hackensack, Bergen co

Washington, Warren co

Woodbury, Gloucester co

Salem, Salem co

Madison, Morris co

Paterson, Passaic co

Ridgewood, Bergen co

New Brunswick, Middlesex co

Red Bank, Monmouth co

Burlington, Burlington co

Greenville, Hudson co

Rahway, Union co

Greenville, Hudson co

Masonville, Burlington co

Jersey City, Hudson co

South Vineland, Cumberland co

Watsessing, Essex co

Beverly, Burlington co

West Hoboken, Hudson co

Millville, Cumberland co

Newark, Essex co

Mount Holly, Burlington co

Merchantville, Camden co

Madison, Morris co

Berlin, Camden co

Swedesboro, Gloucester co

Elizabeth, Union co

Lodi, Bergen co

Passaic, Passaic co

Lodi, Bergen co

Green Village, Morris co

Fairview, Bergen co

Cape May, Cape May co

Little Silver, Monmouth co ${ }^{\circ}$ 
Koch, E. J. $f$

Krause, George $f$

Kuehl, H. $f$.

Kumerle, J. U. $f$.

Lampe, Mrs. A. T. F.

Lanahan, John $f$.

Leahey, Henry $f$.

Lehman, N. G. $f$.

Lester, Benjamin $f$

Mr. Lenly $f$

Lippincott, James $n$

Littejohn, James $f$

Littejohn, James, Jr. $f$.

Lovett, John T. n

Marple, C. C. $f$

Marsh, A. B. $f$

Maul, J. A. $f$

Mawer, William $f$

May, John $f$.

Meadow Brook Nursery $n$

Miller, John $f$

Millinger, Henry

Mitchell, Thomas

Mitchie, Alexander $f$

Mueller, Aloys $f$.

Muzzy Brothers $n$

McBean, A. S. $f$.

McCallum, Donald $f$

McCullough, James $f$.

McGall, Henry J. $f$

McIntyre, James $f$

McKay, Donald $f$

Newbold, F. $f$

Nichol, J. $f$.

Nichols, P. $f$.

Norton, Henry A. $n$

Norton, Wyckoff $f$

Olm, Peter $f$

Parry, William $n$.

Paulsen, J. $f$

Perkins, John $n$

Petit, Clark $n$

Pierson, John $f$

Purdue, R. $f$

Quinlan, William $f$

Raddican, Thomas $f$.

Ransom, J. O. $n$.

Rassbach, John $f$.

Rau, N.

Read, Charles $f$.
West Hoboken, Hudson co

Jersey City, Hudson co

West Hoboken, Hudson co

Newark, Essex co

Jersey City, Hudson co

Princeton, Mercer co

Elizabeth, Union co

Leonia, Bergen co

Boonton, Morris co

West Hoboken, Hudson co

Mt. Holly, Burlington co

Chatham, Morris co

Chatham, Morris co

Little Silver, Monmouth co

Gloucester, Gloucester co

Perth Amboy, Middlesex co

Sea Isle City, Cape May co

Newark, Essex co

Summit, Union co

Englewood, Bergen co

Bergen, Hudson co

Merchantville, Camden co

Morristown, Morris co

Montclair, Essex co

New Brunswick, Middlesex co

Paterson, Passaic co

Lakewood, Ocean co

Camden, Camden co

Madison, Morris co

Orange, Essex co

Morristown, Morris co

Leonia, Bergen co

East Newark, Essex co

Madison, Morris co

Bridgeton, Cumberland co

Hightstown, Mercer co

Hightstown, Mercer co

Newark, Essex co

Parry, Burlington co

West Hoboken, Hudson co

Moorestown, Burlington co

Salem, Salem co

Jersey City Heights, Jersey City

East Orange, Essex co

Madison, Morris co

Madison, Morris co

Hammonton, Atlantic co

Bloomfield, Essex co

West Hoboken, Hudson co

Orange, Essex co 
Reagan, James $f$

Reid \& Roehrs $f$

Reisenberger, L. $f$

Rhoades, Charles $f$

Ribsam, Carl \& Son $f$.

Rodgers, S. E., \& Son $n$

Roehrs, Julius $f$.

Roem, A. $f$.

Rossow, J. C. $f$

Rowbach, A. \& G.

Rulon, B. C. $n$

Rusling, Mrs. Mary $f$.

Schrader, C. F. Jr. $f$

Schrœter, A. C. $f$.

Schweinhagen, C. $f$

Shearman, Major \& Co. $n$

Shim, Wardell $f$.

Shultz, Alexander $f$

Shultz, G. A. $n$

Slaughter, F. J. $f$.

Smalley, I. M. $u$.

Snyder, George B. $f$

Spears, Charles $f$.

Starn, C. W. $n$.

Steigauf, Philip $f$.

Stillwell, H. $f$.

Stokes, Ezra $n$.

Sturtevant, E. D. $f$

Such, George $f$.

Thorley, Mrs. Charles

Thurston, C. $f$.

Torrey, A. J. $f$.

Towell, Joseph $f$.

Townsend, James $f$

Turnley, C. W. $f$.

Ulmer, W. M. $n$.

Vail, A. R. $n$

Vandeventer, James $n$.

Vanneman, Miss Helen $f$.

Van Reyper, E. J., \& Bro. $f$

Van Syckle, S. W. $n$

Voght, William $f$.

Voigt, Carl $f$

Vreeland, S. B. $f$.

Wainwright, George $f$.

Ward, P. J. $f$

Wehrly, Mrs. A. $f$

Welch, George $f$.

White, John $f$.
Camden, Camden co

Madison, Morris co

Bergen, Hudson co

Moorestown, Morris co

Trenton, Mercer co

Mount Holly, Burlington co

270 2d St., Jersey City

Union, Union co

Jersey City Heights, Hudson co

Pemberton, Burlington co

Mullica Hill, Gloucester co

Hackettstown, WVarren co

Bergen Point, Hudson co

Bayonne City, Hudson co

Camden, Camden co

Lakewood, Ocean .

Mt. Holly, Burlington co

Phillipsburg, Warren co

Jamesburgh, Middlesex co

Madison, Morris co

Roadstown, Cumberland co

Fair Haven, Monmouth co

Salem, Salem co

Parry, Burlington co

Egg Harbor City, Atlantic co

Crosswicks, Burlington co

Berlin, Camden co

Bordentown, Burlington co

South Amboy, Middlesex co

Jersey City, Hudson co

Ridgewood, Bergen co

Madison, Morris co

Paterson, Passaic co

Berlin, Camden co

Haddonfield, Camden co

Milford, Hunterdon co

Quakertown, Hunterdon co

Princeton, Mercer co

Woodbury, Gloucester co

Jersey City, Hudson co

Rocky Hill, Somerset co

Wenonah, Gloucester co

Newark, Essex co

Jersey City, Hudson co

Trenton, Mercer co

Bloomfield, Essex co

Somerville, Somerset co

Madison, Morris co

Jersey City, Hudson co 
Wiegand Bros. $f$.

Wilcox, J. $n$.

Williams, C. \& J. C. $n$

Williamson, William $n$

Willson, Pierpont $n$.

Witheridge, Charles $f$.

Woodruff, W. B. $f$

Woolson \& Co. $f$.

Wyckoff, W. H. $f$
West Hoboken, Hudson co

Vineland, Cumberland co

Mount Clair, Essex co

New Durham, Hudson co

Vineland, Cumberland co

Newark, Essex co

Westfield, Union co

Passaic, Passaic co

Asbury Park, Monmouth co

\section{NEW MEXICO.}

Garcia, Felipe \& Co. $n$

Thompson \& Bull $n$
Albuquerque, Bernatillo co

San Lorenzo P. O., Santa Rita, Grant co

\section{NEW YORK.}

Aldrich, Alexander $f$. Alexander, John W. $f$. Allen Bros. $n$

Allen, C. L., \& Co. $n$. Allen, William S. $f$. Alverson, J. C. $f$. Ammann, Charles $f$

Amos, W. $f$.

Ancock, J. $f$

Anderson, Henry S. $n$ Ankem, F. G. $n$

Anthony, Horace $n$

Arnold, Charles $f$

Arnold, Joseph $f$.

Arnold's Sons, I. H. n

Ascott, Thomas $f$.

Atwood, D. F., \& Co. $n$ Babcock, I. H. $n$.

Bacon, Edmund $n$

Baer \& Reisig $f$.

Bailey, Frederick B. $n$
White's Corners, Buffalo, Erie co I3 Mandeville St., Utica, Oneida co

Palmyra, Wayne co

Garden City, Queens co

940 Broadway, New York city

Poughkeepsie, Dutchess co

Cor. 7 th Ave. and West I2oth St., and

254 W. I 3 Ist St., New York city

IVoodside, Queens co

464 Whitesboro St., Utica

Union Springs, Cayuga co

Troy, Rensselaer co

Union Springs, Cayuga co

I 2 I 2 d Ave., New York city

Flatbush, Kings co

Benton Centre, Yates co

Flatbush, Kings co

I64 Main St., Geneva, Ontario co

Lockport, Niagara co

Westbury, Cayuga co

New York city

Geneseo, Livingston co 
Bailey, Harriet $f$.

Baily, John W. $f$.

Baker, Charles F. $f$

Ball, John $f$.

Ball, W. $f$

Barilaite, M. H. $n$

Barrett, Clarence T. $n$.

Batchelor, John $f$.

Bebus, Jacob G. $f$

Bechamp, Joseph $f$

Bechamps, John B. $f$.

Bechamps, John G. $f$.

Beckwith, H. \& J. K. n

Belding, Chester $f$

Bennett, William $f$

Benz, Albert $f$.

Bingham, J. W. $n$

Binley, Arthur J. $f$

Bishop, James $f$.

Blauvelt, A. T., \& Son $n$

Blissard, Frank $n$

Bogue, J. T. $n$

Bogue, N. $"$

Boice, Edwin L. $n$

Borrman, Emil $f$.

Bouck, D. $n$

Bowden, H. J., \& Co. n

Bowden, H. J., \& Co. $n$

Bowman, Thomas W. n

Boyce, George $f$.

Bradley, C. H. $n$.

Bradshaw, John $f$

Brandow, John I. $n$

Brauer, John $f$

Breese, H. G. $n$.

Bridgeman, Alfred $f$.

Brill, Francis $n$.

Brocksbank \& Allen $f$

Bronson \& Hopkins $n$.

Brown, E. M. $n$.

Brown, Everett $n$.

Brown, David $n$.

Brown, G. $f$.

Brown, James $f$

Bryant Bros. $n$

Bryant, Henry C. $n$

Buchanan, Isaac $f$

Buchanan, Isaac $f$
8I 5 8th Ave., New York city

Plattsburgh, Clinton co

57 Cornelia St., Utica, Oneida co

Jamaica, Long Island

Spuyten Duyvil, Station S, New York city

- P. O. Box 3392, New York city

West New Brighton, Richmond co

14 Liberty St., Utica

958 Broadway, New York city

Bayside, Queens co

${ }_{5} 6$ 3d Ave., New York city

East 85th St., near Avenue B, New

York city

Brighton, Monroe co

Middletown, Orange co

Flatbush, Kings co

Douglaston, Queens co

Marlborough, Ulster co

221 Clinton Ave., Albany

ro24 North St. Paul St., Rochester

Blauveltville, Rockland co

West Brighton, Monroe co

Batavia, Genesee co

Batavia, Genesee co

Madalin, Dutchess co

332 High St., Buffalo, Erie co

Lockport, Niagara co

Brighton, Monroe co

Rochester, Monroe co

Rochester, Monroe co

48 Kemble St., Utica

Gerry, Chautauqua co

Sing Sing, Westchester co

Coxsackie, Greene co

37 North St., Buffalo, Erie co

Hoosick, Rensselaer co

37 Eåst I9th St., New York city

Riverhead, Suffolk co

Hudson, Columbia co

39 Seneca St., Geneva, Ontario co

Ellisburg, Jefferson co

Bluff Point, Yates co

Lockport, Niagara co

Long Island City, Queens co

Sing Sing, Westchester co

Dansville, Livingston co

Buffalo, Erie co

407 5th Ave., New York city

Long Island City, Queens co 
Buongiorno, R. $f$

Burgevine, Charles $n$. Burgevine, Valentine $n$ Burnham, Sarah J. $f$. Burns, Alexander S. $f$. Burns, Alexander S. $f$. Burrow, J. S. $n$ Burt, Albert $f$

Bussard, Henri $f$. Busse, William $f$. Butler, E. A. $f$. Butler, Henry $f$. Butler, P. W. $n$. Canary, Thomas $n$ Campbell, R. J. $f$. Carey, John \& Edward $n$ Carpenter, S. W., \& Son $n$. Carr, Thomas $\mathrm{F}$. Carson, William H. $f$. Carter, Henry $f$.

Case, B. J. $n$

Cass, W. H.

Caywood, A. J., \& Sons $n$

Chapman \& Austin $n$.

Chapman, J. C. $f$.

Chapman, William I. $n$

Charlton, John $f$.

Chase Bros. $n$

Chase, R. G., \& Co. $n$.

Chatfield, A. F. $f$

Chielester, W. P. n

Childs, J. Lewis $n$

Christensen, Chris. $f$.

Clark, A. G.

Clark, David, \& Son $f$

Clark, E. P. $n$

Clark, Thomas $f$.

Clayton, Thomas $f$

Cockburn, Samuel $f$

Cole, C. S.

Coleman, Antholy \& Co. $n$.

Condon, John $f$

Cook, A. F. $n$

Copin, August A.

Cordier, Mrs. IVilhelmina $f$

Corney, H. G. $n$.

Corning, W. B., Jr. $f$.
Boulevard C, West 8oth St., New York city

Kingston, Ulster co

Kingston, Ulster co

245 West 129th St., New York city

840 6th Ave., New York city

Woodside, Queens co

Fishkill, Dutchess co

Cor. Warren and Washington Sts., Syracuse, Onondaga co

Yonkers, Westchester co

Broadway, near Fowler Bath, Albany 3d and Ontario Sts., Niagara Falls 9 8 Broadway, New York city

East Penfield, Monroe co

Hamburgh Turnpike, Buffalo

Forrest Hill Cemetery, Utica

Rochester, Monroe co

Harrison, Westchester co

596 6th Ave., New York city

I 4 Chambers St., New York city

Newburgh, Orange co

Sodus, Wayne co

Geneva, Ontario co

Marlborough, Ulster co

Fayetteville, Onondaga co

I I I Water St., Newburgh, Orange co

Hartford, Washington co

University Av., Rochester, Monroe co

Rochester, Monroe co

I64 Main St., Geneva, Ontario co

66 Chestnut St., Albany

Smith's Landing, Greene co

Queens, Queens co

Delaware Ave., Buffalo, Erie co

Marlborough, Ulster co

Boulevard C, West 78th St., New

York city

Dansville, Livingston co

Brooklyn, W. D., Kings co

90 Richmond Ave., Buffalo, Erie co

Woodlawn, Station S., New York city

Spencerport, Monroe co

Lewis St., Geneva, Ontario co

Brooklyn, W. D., Kings co

Clinton Hollow, Dutchess co

223 6th Ave., New York city

College Point, Queens co

Cornwall-on-Hudson, Orange co

Sparkhill, Rockland co 
Cornish, WV. H., \& Co. n

Corwin, George $f$

Cotes, Byron S. $f$

Covey, Alvah $n$

Covey, E. T. $n$

Cowan, A. D., \& Co. $f$

Cowen, James $f$.

Craik, Joseph, \& Son $f$

Crissey, J. H. $n$

Cropp, Charles $f$.

Crosby, Samuel D.

Crossman Bros. $f$.

Crowe, Peter $f$.

Crystal, Matthew $f$

Cullen, Hugh $f$.

Dagge, Edward $n$

Dagwell, S. L. $n$.

Dailledouze Bros.

Dards, Charles A

Darman, C. G. $f$.

Davidson, Thomas

Davies, Samuel $n$

Davis \& Sons $n$.

Day, J. G. $f$.

Dean, David $f$

Dean, James $f$

Defries, Henry $f$.

Degnelle, Adolph $f$

Delmour, Lawrence $f$.

De Pew, Tunis $f$.

De Veer, J. A. $n$.

Dietz \& Holman $f$

Dietzenberger Bros. $f$.

Dihm Bros.

Dingwall, John $f$.

Dixon, J. B. $n$.

Dobbertin, J. Jr. $f$

Dobbins, S. $f$

Dole, Charles $f$.

Donadi, M. $f$.

Donnelly, R. J. $f$.

Donohue, Frederick $f$.

Doran, Charles L.

Dorval, Victor S. $f$

DuBois, Marcus D. $n$.

Dunscombe, J. Howard

Durbin Bros. $n$
Newburg, Orange co

Havana, Schuyler co

West Io4th St., near 9th Ave., New York city

Penfield, Monroe co

Penfield, Monroe co

I 4 Chambers St., New York city

Yonkers, Westchester co

Culver Road, Rochester

Fredonia, Chautauqua co

297 Hoyt St., Brooklyn

Io6 Broad St., New York city

503 Monroe Ave., Rochester

73I Genesee, Utica, Oneida co

9I 8 Broadway, New York city

240 6th Ave., New York city

ochester, Monroe co

Utica, Oneida co

Flatbush, Kings co

Cor. 44th St. and Madison Ave., New York city

Batavia, Genesee co

Knox S. Madison Ave., Albany

72 Perkins Ave., Utica, Oneida co

Fredonia, Chautauqua co

307 S. Salina St., Syracuse, Onondaga co

Long Island City, Queens co

Bay Ridge, Kings co

587 Plymouth Ave., Rochester

IoI West I4th St., New York city

I 500 3d Ave, New York city

Nyack, Rockland co

3I 8 Broadway, New York city

66 Carmine St., New York city

Bay Ridge, Long Island

408 West I roth St., New York city

Iozo Broadway, Albany

Waterloo Road, Geneva, Ontario co

595 St. Joseph St., Rochester

Buffalo, Erie co

Lockport, Niagara co

Long Island City, Queens co

Rochester, Monroe co

I I East I4th St., New York city

813 6th Ave., New York city

Woodside, Queens co

Newburgh, Orange co

Sing Sing, Westchester co

Weedsport, Cayuga co 
Dutcher, W. C. $n$

Dutchess Nurseries Co. $n$

Eckard, Philip $f$.

Edwards, W. H., \& Co. $f$

Eger, J. C. $n$

Ekirch, Alphonse $f$

Elletson, Jacob $f$.

Ellis, Robert $f$.

Ellis, William H.

Elwanger \& Barry $n$

Elwanger, Frank E. $n$.

Esch, John $f$

Escott, T. $f$.

Fellows, Wm., \& Son $n$

Ferries, W. H., Jr. $n$.

Ferris, W. L. $n$

Field, H. N. $n$

Finn, John $f$

Fish, George T. $n$

Fitzelle, F. A. $f$.

Fitzgerald, James $f$

Fitzpatrick, Mrs. A. C.

Fitzsimmons, Michael $f$

Flechter, A. B. $n$.

Foote \& Co. $f$.

Force, T. S. $n$

Ford, W. $n$.

Forrest, William $n$

Foster, P. H. $n$.

Foulis, David $f$

Franck, Henry $f$.

Frank, T. F. $f$.

Frederick, G. W. n

Frederick, Victor $n$

Freund, Charles

Fricker \& Clark $f$

Friedlander, Mrs. L. $f$.

Frielingsdorf, Anna $\mathrm{H} . \quad f$

Frielingsdorf, Francis M. $f$.

Frielingsdorf, John $f$

Frith, Thomas n

Frost \& Co, $n$

Frost, Adolph $f$.

Frost, E. A. $n$

Frost, E. D. $n$

Fulton, Andrew $n$

Fulton, H. P. $n$.
Naples, Ontario co

Poughkeepsie, Dutchess co

College Point, Queens co

Dunkirk, Chautauqua co

Schenectady, Schenectady co

I 197 Broadway, New York city

Io6 Franklin St., Auburn, Cayuga co

White Plains, Westchester co

4I 7 3d Ave., New York city

Mount Hope Nurseries, Rochester, Monroe co

Rochester, Monroe co

East 17oth St., near Boston Road, New York city

Flatbush, Kings co

Penfield, Monroe co

Poughkeepsie, Dutchess co

Poughkeepsie, Dutchess co

West Oneonta, Otsego co

East 67th St., near 2d Ave., New York city

Rochester, Monroe co

Yonkers, Westchester co

Astoria, Long Island, Queens co

1213 Broadway, New York city

74 Fulton St., New York city

Marlboro, Ulster co

Ithaca, Tompkins co

Newburgh, Orange co

Fishers, Ontario co

Ridgeway, Orleans co

Babylon, Suffolk co

I 482 Broadway, New York city

8I 5 Broadway, New York city

95 Gelston St., Buffalo, Erie co

Wellsville, Allegany co

Long Island City, Queens co

Rye, Westchester co

Poughkeepsie, Dutchess co

New York city

896 3d Ave., New York city

I 384 3d Ave., New York city

539 3d Ave., New York city

Brooklyn

I6 Frost Ave., Rochester

Cortland, Cortland co

Rochester, Monroe co

Havana, Schuyler co

Johnstown, Fulton co

Gerry, Chautauqua co 
Gabrielson, G. $f$.

Gamble, J. B. $n$.

Gard, Joseph $f$

Gardner, Rowland W.

Garreston, G. R. n

Geib, George $f$.

Geib, Valentine \& Son $f$

Germond, W. C. $f$

Gibson \& Towell $f$

Glen Bros. "

Glœcker, L., Mrs. $f$

Goble \& Vredenburg $n$

Golsner, Gabriel $f$

Gordon, Frederick $f$.

Gorham, J. R., Jr. $f$

Grandall \& Morrison $n$

Graves, B. M. $n$

Grecht, Ernest $f$.

Green, Mrs. Amanda $f$

Green, Charles A. $n$.

Greene, W. W., \& Son n

Gregory, Hiram $n$

Grinton, David $f$.

Griswold, W. H. n

Gurks, Harvey $n$.

Guenther, John F.

Guterdeng, H. $f$.

Haag, Charles $f$.

Haake, Christian $n$

Haggerty, Mrs. R. V. $f$

Hahl, Mrs. $f$

Haines, E. C. $f$.

Haines, Lemuel $f$

Hale, Mrs. J. $f$

Hall, William A. $n$

Hallock, V. H., Son \& Thorpe

Hamilton, John S., Jr., $f$

Hammer, J. M. $f$.

Hammond \& Hunter

Hammond, A. $n$.

Hammond, J. $n$.

Hammond, W. S. $f$

Hanft Bros. $f$

Hanft, George $f$.

Hanley, C. L. $f$.

Hannor \& Parker $n$

Hansen, Frederick $f$

Harrison, Joseph $n$

Hart, A. $f$.
82 I Broadway, New York city

Oak Orchard, Orleans co

Flatbush, Kings co

Fredonia, Chautauqua co

Flushing, L. I., Queens co

300 2d Ave., New York city

509 Grand St., New York city

Sparkhill, Rockland co

509 Madison Ave., New York city

Rochester, Monroe co

Albany Rural Cemetery Ave., Albany

Rochester, Monroe co

College Point, Queens co

26 Broad St., New York city

79 Water St., Newburgh, Orange co

Troy, Rensselaer co

Farmer Village, Seneca co

215 East 14 th St., New York city

Elmira, Chemung co

Clifton, Monroe co

Watertown, Jefferson co

Lockport, Niagara co

67 West Chippewa, Buffalo, Erie co

Rose, Wayne co

Oswego, Oswego co

255 Park Ave., Rochester

Boulevard C, West 95th St., New York city

53 Little West 12th St., New York city

Port Chester, Westchester co

Poughkeepsie, Dutchess co

Flatbush, Kings co

Bedford Station, Westchester co

Peekskill, Westchester co

I 74 Pearl St., Buffalo, Erie co

Au Sable Forks, Essex co

Box A, Queens, Queens co

7 I Broadway, New York city

359 West Ave., Rochester

5I West 3oth St., New York city

Geneva, Ontario co

Geneva, Ontario co

Carthage, Jefferson co

$2245^{\text {th }}$ Ave., New York city

795 Broadway, New York city

Hornellsville, Steuben co

Mt. Morris, Livingston co

I 4 East I I th St., New York city

Rochester, Monroe co

Flatbush, Kings co 
Hart, Charles S. $f$

Hart, James $f$

Hasselblad, William $f$

Hawkins, G. W.

Hawkins, J. R. V. n .

Hay, J. S. $f$.

Hays, R. H., \& Co. $f$.

Haywood, Thomas E. n

Hefele, Simon $f$.

Heinrich, Julius J. $f$.

Henderson, Peter, \& Co. $f$.

Henderson, John $f$

Hendricks, James $f$

Hennessey, Michael $f$.

Herod, William $f$

Herring, George E. $f$.

Heyardt, August

Hicks, Isaac, \& Sons $n$

Hicks, Sarah M. $f$.

Higgins, William G. $f$.

Hoag \& Cox $f$

Hoag, John $f$

Hodgson, John M. $f$

Hoelzle \& Grassmuck

Hof heimer, Solomon $f$

Hofmeister, Charles F. $f$

Holley, E. $f$

Holwede, George $f$.

Hood, Lewis M.

"H. E. Hooker Company" $f$

Hooper, John $f$.

Hopkins, L. D. $n$.

Horn, Oliver $n$.

Hosmer, S. A. $n$.

Hubbard, Elisha C. $n$.

Hubbard, T. S. $n$.

Hubbart, J. E. $n$.

Huckins, Edwin

Hudson, Mrs. A. $f$

Hughes, James F. $f$.

Hull, N. S. $n$

Humphrey, C. B.

Husted, S. B.

Hyatt, N. R. $n$

Irvin, William $\mathrm{H}$.

Isaacs, Abraham L. $f$.

Jaeger \& Sons $f$.
Watertown, Jefferson co

I 7 West 3oth St., New York city

48 West 3 Ist St., New York city

Middle Hope, Orange co

Goshen, Orange co

Oneida, Madison co

Cor. Ist and Chamber St., Newburgh,

Orange co

Pittsford, Monroe co

I06I North 3d Ave., New York city

Brooklyn, W. D., Kings co

35 and 37 Cortlandt St., New York city

Flushing, L. I., Queens co

36 N. Pearl St., Aılbany

Poughkeepsie, Dutchess co

I389 Atlantic Ave., Brooklyn

Iog West 4Ist St., New York city

Cor. Reid Ave. and McDonough St.,

Brooklyn

Westbury Station, Queens co $4064^{\text {th }}$ Ave., New York city

69 Barclay St., New York city

4I 3 Fulton St. and 357 Broadway, Troy

Newburgh, Orange co

$5455^{\text {th }}$ Ave., New York city

I I 6 3d Ave., New York city

3 East 4th St., New York city

299 8th Ave., New York city

Hudson, Columbia co

27 East Main St., Rochester

I 4 West 34th St., New York city

"Hooker Nurseries," Rochester

Gloversville, Fulton co

Cortland, Cortland co

Lyons, Wayne co

Clifton, Monroe co

Water Valley, Erie co

Fredonia, Chautauqua co

Brighton, Monroe co

Mt. Vernon, Westchester co

2 W. Lake Ave., Auburn, Cayuga co

107 E. I27th St., New York city

Norwich, Chenango co

Walesville, Oneida co

Blauveltville, Rockland co

Pultney, Steuben co

69 Broadway, New York city

300 West 28th St., New York city

I80 Ave. B, New York city 
Jahn, Augustus $f$. Jenkins, David $f$. Jenkins, Richard F. $f$ Jenkins, Thomas B. $n$. Jennings, Richard $f$. Jervis, G. S. $f$ Johnson, J. T. $f$. Johnson R. $n$ Johnson, William $n$ Johnson, William G. $f$. Jones, A. N. $n$. Jones, Morris R. $f$ Jones \& Rouse $n$. Jonston, Abram $f$ Joosten, Christian H. $f$ Joslin, George H. n Josselyn, George S. $n$. Kadletz, John $f$. Katoll, Frederick F. $f$. Keene, Peter A. $f$ Keenan, Ernst $f$. Keitsch, Henry $f$ Keller, John B. $f$. Keller, John M. $f$ Kelsey, Frederick IV. n Kennedy, T., \& Sons $n$ Keyes, John $f$. Keyser, Mary $f$. Kind, Charles, \& Son $f$

King \& Murray $n$ King, William H. n

Kirke, G. W. $n$ Kirkpatrick, Edward $f$ Klodt, W. R. $f$ Klunder, Charles F. $f$. Knickman, Charles Koch, Charles E. $f$

Koch, John $f$ Kœvend, Arthur Kohl, August $f$. Kopp, Jacob $f$ Korn, Julius $f$ Krauter \& Noeding $f$. Kretschmar Bros. $f$ Krick, Charles $f$. Krombach, Charles $f$. Kruger, Charles E. $f$.
108 Broadway, Brooklyn I653 Fulton St., Brooklyn 682 8th Ave., New York city

Rochester, Monroe co

Poughkeepsie, Dutchess co

Maspeth, Queens co

Kingston, Ulster co

Shortsville, Ontario co

East Penfield, Monroe co

Ithaca, Tompkins co

Le Roy, Genesee co

Rome, Oneida co

Rochester, Monroe co

Newburgh, Orange co

22 New Church St., New York city

Long Year, Ulster co

Fredonia, Chautauqua co

New Dorp P. O., Richmond co

I 2 I Linwood Ave., Buffalo, Erie co

I 54 Reed St., New York city

408 Myrtle Ave., Brooklyn

I 3 Clinton Market, Buffalo, Erie co 361 South Ave., Rochester

Bay Ridge, Kings co

208 Broadway, New York city

Dansville, Livingston co

9I 8 Broadway, New York city

Washington Market, New York city 300 West 3 Ist St. and 300 West $44^{\text {th }}$

St., New York city

Flushing, Queens co

232 Mount Hope Ave., Rochester,

Monroe co

26 Burling Slip, New York city

Sing Sing, Westchester co

342 Myrtle Ave., Brooklyn

907 Broadway, New York city

764 3d Ave., New York city

Boulevard N, West 8gth St., New York city

183 Essex St., New York city

Parkville, Kings co

13 Avenue A, New York city

120 Troutman St., Brooklyn

215 East 14th St., New York city

I7 Avenue A, New York city

Flatbush, Kings co

I097 Broadway, Brooklyn, Kings co

Brooklyn, WV. D., Kings co

496 Monroe Ave., Rochester 
Krull, G. H. $f$

Kuhn, Jacob $f$

Kullman, Martin $f$

Kunzel, Christopher $f$.

Lane, Samuel Y. $f$

Landon, IV. N. - $f$.

Lang, Louis $f$

Lang, Theodore J. $f$.

Larochelle, Amelia $f$.

Latour, Philip $f$.

Lawrence, Thomas $n$.

Leach, Bailey $f$

Le Clare \& Herrick $n$.

Le Clare, James F. $n$.

Lee, John $f$.

Lehde, J. G. n

Lehmann, Abraham $f$.

Le Moult, Adolphus $f$.

Le Mouit, Emil $f$.

Lewis, E. B. $f$.

Lichtenberger, Max $f$.

Lindmark, Charles G. $f$

Link, Henry $f$.

Little, William S. $n$

Long Bros. $f$.

Long Bros. $f$.

Long, Charles $f$.

Long, David N. $n$

Long \& Houghton $f$

Lorentz, John $f$.

Lorenz, Frank J. n

Love, J. W. n

Loveland, Levi A $f$

Lucke, Adolph $f$.

Lurk, John $f$.

Lutts, Henry

Lutz, George $f$

Lynch, John H. $f$.

Lynch, M. J. $f$

Lyon, Harrison A. $n$.

Mallon, James $f$.

Mallon, Peter $f$

Mann, E. $n$.

Mansfield, Thomas $f$.

Marc, Gabriel $f$.

Marinor, George L. $f$.

Marsden, Joseph $f$
129 8th St., Brooklyn

Nyack, Rockland co

College Point, Queens co

Ridgewood, Long Island

Cor. South and Miller Sts., Utica

Syracuse, Onondaga co

250 N. Division St., Buffalo, Erie co 302 West 35th St., New York city

259 8th Ave., New York city

503 Washington St., Buffalo, Erie co

Ogdensburg, St. Lawrence

Long Island City, Queens co

Rochester, Monroe co

Rochester, Monroe co

Hastings-on-the-Hudson, Westchester co

Gardenville, Erie co

296 Grand St., New York city

I 72 Bowery, New York city

436 4th Ave., New York city

Lockport, Niagara co

33 Avenue A, New York city

254 3d Ave., New York city

25 Washington Mrkt., Buffalo, Erie co

407 East Ave., Rochester, Monroe co

Niagara St., Buffalo, Erie co

Williamsville, Erie co

Rochester, Monroe co

Williamsville, Erie co

7216 th Ave., New York city

167 Broadway, Brooklyn

St. Nicholas Ave., N. W., I 5 Ist St.,

New York city

Geneva, Ontario co

Newark, Wayne co

Ithaca, Tompkins co

College Point, Queens co

Youngstown, Niagara co

I4 Avenue A, New York city

141/2 2d Ave., New York city

Poughkeepsie, Dutchess co

Brighton, Monroe co

405 Fulton St., Brooklyn, W. D.,

Kings co

473 Fulton, Brooklyn

Alexander, Genesee co

Lockport, Niagara co

Woodside, Queens co

I80 South St., New York city

Far Rockaway, Queens co 
Marvin, D. S. $n$.

Mathesen, Charles W. $f$

Matthews, William $f$

Mattison, E. C. $n$.

Mattoon, A. C. $f$.

Maxwell, T. C., \& Bro. $f$

Meindel, George $f$

Mellin, J. P. $n$

Menand, L., \& Son $n$.

Mendahl \& Holwede $f$

Merritt, Mrs. M. C. $f$.

Merritt, S. H. $f$

Messeburg, Gustavus $f$

Metz Bros. $f$.

Metz, Henry J. $f$.

Meyerholz \& Blum $f$.

Midler, Mrs. P. P. $f$

Millany, Frank $f$.

Miller, A. J. $f$.

Miller, Henry $f$.

Milley, James $f$.

Milliott, Pierre $f$.

Mills, Myron H. $f$

Moody, E., \& Sons $n$.

Morey \& Sweet $n$.

Morf, C. $n$

Morgan, James $n$.

Morris, J. A. $f$.

Morris, J. W. $f$

Moulson, Samuel $n$

Muller, John $f$

Mullins, John T. $n$

Munch, Herman $f$

Myers, John

Myers, John H. $n$.

McAllister, Francis E. $f$

McClelland, Aleck $f$

McConnell, Alexander $f$

McConnell, Andrew $f$.

McDonald \& Hanft $f$.

McElroy, Thomas $f$.

McFarland, L. W. $f$.

McGarry, John $f$.

McGomay, John $f$

McGovern, Patrick $f$.

McGraw, D. C. $f$.

McKelvey, IVilliam G. n

McKinstry, R. W. $n$.

Neal, Mrs. Lena $f$.
Watertown, Jefferson co

673 6th Ave., New York city

Cor. York and Green Sts., Utica

Jacksonville, Tompkins co

Oswego, Oswego co

Castle St., Geneva, Ontario co

54 Avenue A, New York city

Castle St., Geneva, Ontario co

Menand Station, Albany, Albany co

92 East Main St., Rochester

I01 Oneida St., Utica

Geneva, Ontario co

Flatbush, Kings co

East 83d St., near 3d Ave., N. Y. city 201 East $125^{\text {th }}$ St. and East 170 North

Washington Ave., New York city

77 3d Third Ave., New York city

Syracuse, Onondaga co

Bayside, Queens co

East New York, Kings co

East New York, Kings co

346 Maston St., Buffalo, Erie co

Yonkers, Westchester co

Binghamton, Broome co

Lockport, Niagara co

Dansville, Livingston co

Brewster, Putnam co

P. O. Box 23, Auburn, Cayuga co

Warsaw, Wyoming co

Utica, Oneida co

Rochester, Monroe co

9o Clinton Market, New York city

Rhinebeck, Dutchess co

266 East 4th St., New York city

912 6th Ave., New York city

Saratoga Springs, Saratoga co

3I Fulton St., New York city

Dobbs Ferry, IVestchester co

7296 th Ave., New York city

West New Brighton, Richmond co

63 West I4th St., New York city

3o West Broadway, New York city

New Hartford, Oneida co

22 Costar St., Rochester

28 Locust St., Rochester

455 2oth St., Brooklyn

Binghamton, Broome co

Geneva, Ontario co

Hudson, Columbia co

79 East Broadway, New York city 
Nellis, A. C., \& Co. $f$.

Nelson, J. $n$.

Newborn, A. M. $f$

Newdale, Albert $f$

Newton, J. M. $n$.

Nichols, George $n$

Nickel, George $f$.

Nilsson, William $f$

Norris, J. F. n

Northrup, B. D. $f$.

Nugent, John B. $f$

Nugent, J. J. $f$

Nungesser, Henry $f$

Nurge, Christopher $f$.

O'Connor, James J. $f$.

Opry, William G. $f$.

Palmer, Elisha O. $n$

Palmer, Gridley $n$

Palmer, S. C. $n$.

Palmer, W. J. $f$.

Palmer, W. J. $f$.

Parshall, John $n$.

Parsons, E. W., \& Co. $n$.

Parsons, Robert B., \& Co. n

Parsons \& Sons Co. (Limited)

Paterson, George

Patrick, A. $f$.

Patty, D. H.

Paulsen, James O. $f$

Pearson, W. \& P. $n$

Pearson \& Waite $f$

Pease, L. B. $f$

Peattie, E. M., \& Bro. $f$

Peck, John B. $f$

Peck, R. M. $n$

Peek, J. Y. $f$.

Perkins, John J.

Perry, F. L. $n$

Peterson, Emil $f$.

Pfeiffer, Anselm $f$

Pfordts, J. G., \& Sons $f$

Phillips, John V. $f$

Pickelman, John C. $f$.

Pickelman, Sebastian $f$

Pierson, E. C. $n$.

Pierson, F. R. $f$.

Pilat, C. F. $f$

Platman, J. H. $n$.
Canajoharie, Montgomery co

Brighton, Monroe co

I 39 South St. Paul St., Rochester

27 East Main St., Rochester

Angola, Erie co

Kendall Mills, Orleans co

Stapleton, Richmond co

Woodlawn, New York city

Brighton, Monroe co

Oswego, Oswego co

I2 I $4^{\text {th }}$ Ave., New York city

42 North Main St., Rorhester

I So Lewis St., New York city

Cor. Avenue B and East 22d St., New

York city

I 98 Water St., New York city

403 6th St., New York city

Modena, Ulster co

Groom's Corners, Saratoga co

Turin, Lewis co

306 Main St., Buffalo, Erie co

Lancaster, Erie co

Springwater, Livingston co

West Chester, Westchester co

P. O. Box 603, Flushing, Queens co

$n$. Kissena Nurs's, Flushing, Queens co

New Rochelle, Westchester co

I9 Seward Ave., Auburn

2 I I Exchange St., Geneva, Ont. co

852 Broadway, New York city

Ithaca, Tompkins co

Sparkhill, Rockland co

Lockport, Niagara co

Cor. Dubois and Farrington Streets,

Newburgh

Rye, Westchester co

West Bloomfield, Ontario co

398 Nostrand Ave., Brooklyn

839 6th Ave., New York city

Canandaigua, Ontario co

Brooklyn

45 Ist Ave., New York city

897 and 899 Broadway, Albany

82 Montague St., Brooklyn

280 High St., Buffalo, Erie co

227 Orange St., Buffalo, Erie co

Waterloo, Seneca co

Tarrytown, Westchester co

Sing Sing, Westchester co

Bellona, Yates co 
Plimpton, A. J. $f$.

Porter, George $f$.

Porter, M. L. $n$

Potkora, William $f$

Poynter, Thomas $f$

Price \& Knickerbocker $f$

Pritchard, Henry $f$.

Progatzky, Gustav L. $f$

Purdy, A. M. $n$.

Purdy, H. J., \& Co. $f$.

Purdy, James

Putney, E. D. $n$

Quinlan, P. R., \& Co. n

Ralph, John $f$

Randolph, Edward F. $f$

Raoux, Charles J. $f$.

Rapin Bros. $f$

Rapin Bros. $f$

Rawson, Grove P. $f$.

Raymond Bros. n

Reche, John $f$

Reddy, J. J. $f$

Reddy, Thomas H. $f$. Reed \& Funnell $f$. Reeves, E. A., \& Co. $f$ Reeves, F. H. $f$. Reeves, Robert C. $f$. Reid, Walter $f$

Reinhardt \& Koenig $f$. Remington, W. J. $f$. Rhoades, Emily L. $f$. Richardson, E. F. $n$ Riddock, Elseph $f$

Riesenberger, W. G. $f$. Roche, T $n$

Roeders, Christianna $f$ Roehrs, Theodore $f$. Roemer, Peter, \& Sons $f$ Roesch, Lewis $n$. Rogers Bros. $n$. Rolker, August, \& Sons $f$ Root, James A. $n$. Rose, Alfred $n$ Ross, D. \& P. $f$. Rother, Adam $f$. Rupert, J. O. $n$. Ryan, Timothy $f$. Ryman, George $n$
Hornellsville, Steuben co

Cor. Washington Ave. and Allen, Albany

Fredonia, Chautauqua co

Western Ave., near Ontario, Albany

Brooklyn, W. D., Kings co

So State St., Albany

565 Madison Ave., New York city

79 Avenue A, New York city

Palmyra, Wayne co

Seneca Falls, Seneca

rog IVest 44th St., New York city

Brentwood, Suffolk co

Syracuse, Onondaga co

Saratoga Springs, Saratoga co

64 Pearl St., New York city

296 Pearl St., New York city

Cheektowaga, Erie co

567 Main St., Buffalo, Erie co

Elmira, Chemung co

Penfield, Monroe co

College Point, Queens co

285 Manhattan Ave., Brooklyn

440 Bedford Ave., Brooklyn

Huntington, Suffolk co

68 Cortlandt St., New York city

Watertown, Jefferson co

I 85 Water St., New York city

I286 Broadway, New York city

I48 East 59th St., New York city

204 Putnam Ave., Brooklyn

Brooklyn, W. D., Kings co

Brentwood, Suffolk co

Cor. St. Ann's Ave. and East 139th

St., New York city

733 Broadway, New York city

Newark, Wayne co

447 Canal St., New York city

I 53 West 3Ist St., New York city

Woodlawn, New York city

Fredonia, Chautauqua co

Lyons, Wayne co

44 Dey St., New York city

Skaneateles, Onondaga co

Penn Yan, Yates co

5255 th Ave., New York city

Central Ave., Brooklyn

Pultney, Steuben co

Yonkers, Westchester co

Fredonia, Chautauqua co 
Salter, Bros. $f$

Sambrook, George $f$

Scallen, John W. $f$

Schaeffer, F. J. A. $f$.

Schafer, Jacob $f$.

Scherer, Henry $f$.

Schilowsky, Robert G. $f$

Schlegel, Frederick $f$.

Schmidt, Matthew H. $f$

Schmitt, Antoine

Schmutz, L.

Schultheis Bros. $f$

Schunemann, Christopher $f$

Schwarz, G. F. $f$.

Scofield, S. $n$

Scommodan, Richard $f$

Scott, William $f$.

Seabrick, Louis $f$

Seagrove, William $f$.

Sealey, R. E. \& J. C. $f$.

Searle, S. B. $n$.

Sears, Henry, \& Co. $n$

Seiler, Joseph $f$

Seitzer, Charles F. $f$

Selover \& Wallard $n$.

Semon, John A. $f$

Shackall, James $f$

Shaker Seed Co. $n$

Shaw, J. Austin $n$

Shaw, Samuel R. $f$

Sheriden, Walter F. $f$.

Sibley, Hiram, \& Co. n

Siebrecht, Louis $f$

Siebrecht, W. H.

Siebrecht \& Wadley $f$.

Simonson, Isaac J. $f$.

Singleton, Richard $f$.

Sisson, William $n$

Slover, E. C. $f$.

Smith \& Amos $f$

Smith, Charles A.

Smith, E. $n$.

Smith, E. Y. $n$

Smith, Mrs. G. W. $f$

Smith, Nelson $n$.

Smith, Shelton $n$.
56 State St., Rochester

West Troy, Albany co

268 West 125th St., and 1244 Broad-

way, New York city

386 Broadway, Newburgh, Orange co

Middle Village, Queens co

799 6th Ave., New York city

588 and 906 3d Ave., New York city

260 South Ave., Rochester

236 East 14th St., New York city

White Plains, Westchester co

Flatbush, Kings co

P. O. Box 78, College Point, L. I.

82 Gansevoort St., and 1986 3d Ave.,

New York city

Flatbush, Kings co

Ridgeway, Orleans co

300 IVest 34th St., New York city

Cor. Balcom and Michigan Sts., Buf-

falo, Erie co

Queens, Queens co

I8 I St. James Pláce, Brooklyn

Pearsall's, Queens co

Onondaga Valley, Onondaga co

Cor. Main and Lafayette Sts., Geneva,

Ontario co

89 8th Ave., New York city

84 State St., Utica

Geneva, Ontario co

715 8th Ave., New York city

Bayside, Queens co

Mount Lebanon, Columbia co

Rochester, Monroe co

1023 3d Ave., New York city

26 West 28th St., New York city

I79 East Main St., Rochester

East Hinsdale P. O., Queens co

Long Island City, Queens co

409 5th Ave., New York city

New Springville, Richmond co

1902 Lilian St., New York city

Geneva, Ontario co

Washington St., Geneva, Ontario co

Woodside, Queens co

Clyde, Wayne co

Geneva, Ontario co

Manchester, Ontario co

Brockport, Monroe co

Yorkshire Centre, Cattaraugus co

Pittsford, Monroe co 
Smith, William $f$.

Smith, W. \& T. $n$

Smyth, Edward $f$.

Snook, L. D. $f$

Snyder, Beni $n$

Southwick, T. $n$.

Speiss, Conrad $f$.

Speiss, John $f$.

Spengler, Charles $f$

Sprague, Joseph W. $f$.

Stack, George N.

Staffinger, Philip

Stanley, W. \& W. n

Stark \& Pankok $f$

Stein, Peter $f$

Stevens, F. C. $n$.

Stevens, John $n$.

Steward, C. W. $n$.

Stewardson, J. $f$.

Stewart \& Bishop $f$

Stillwell, Charles R. $f$.

Stoehr, Emma $f$.

Stone, G. C. $n$.

Stone \& Wellington $n$

Stowell, A. D. $n$.

Strickler, F. $f$.

Stuart, C. W. $n$

Studley, S. W. $n$.

Stumpp, George M. $f$.

Suhrer, Eugene $f$

Sunderland, George C. $n$

Taylor, Edward C. $f$.

Taylor, John

Terwilliger, S. F. $f$

Tharp, A. J. $n$

Thomas, A. H. $f$.

Thorburn, James M., \& Co.

Thorburn \& Titus

Thornhill, John $f$.

Thumann, John $f$.

Thurton, John $f$.

Tier, Daniel $f$

Totten \& Shaffer

Towell \& Co. $f$.

Uhl, Jacob $n$

Ulrich, Charles $f$.

Underhill, E. B. $n$
56I Myrtle Ave., Brooklyn

Castle St., Geneva, Ontario co

West 93d St., N. Boulevard, New York city

Barrington, Yates co

Kingston, Ulster co

Rochester, Monroe co

207 Peach St., Buffalo, Erie co

2 I 6 Peach St., Buffalo, Erie co

I 37 Avenue A, New York city

Tottenville, Richmond co

Poughkeepsie, Dutchess co

Landon, near Jefferson St., Buffalo, Erie co

Odessa, Schuyler co

Long Island City, Queens co

9I Ist Ave., New York city

Attica, Wyoming co

Clifton Springs, Ontario co

Newark, Wayne co

West Ave. and Jersey St., Buffalo,

Erie co

North St. Paul n'r Norton, Rochester

Gravesend, Long Island

266 East 4th St., New York city

Dansville, Livingston co

Rochester, Monroe co

Fulton, Oswego co

West Troy, Albany co

Newark, Wayne co

Catskill Station, Columbia co

634 Madison Ave., New York city

I6 Cannon St., New York city

Purdy's Station, Westchester co

Peekskill, IVestchester co

Bayside, Queens co

Saratoga Springs, Saratoga co

Bedford, Westchester co

Ithaca, Tompkins co

I 5 John St., New York city

I 58 Chambers St., New York city

26 West 28 th St., New York city

252 West 23d St., New York city

Mt. Vernon, Westchester co

Woodlawn, N. Y. city

Saratoga Springs, Saratoga co

842 3d Ave., New York city

Dansville, Livingston co

Middle Village, Queens co

Poughkeepsie, Dutchess co 
Valentine, C. A. n

Van Buskirk, John D. $n$

Vanderbilt Bros. Nephew $f$.

Van Der Veur, Peter W. $f$.

Van Duzen, C. L., Nursery Co. $n$

Vetter, Jacob

Vick, James $f$

Von Brown, Adolph F. $f$.

Wagner, Henry $f$

Wakeling, Henry $f$

Walch, S. $f$.

Wales, George S. $n$

Walker, John

Wands, R. J. $f$

Warmt, Frederick E.

Warringer, A. H. n

Webster, William $f$

Weir, James, Jr. $f$

Weir, James, \& Son $f$.

Weis, Frederick

Weld, E. W. $f$.

Wells \& Corwin $n$

Wenisch, Anton $f$

Wheadon, E. D. $f$

Wheeler, Charles

White Bros. $n$.

Whitney, James E. $n$.

Whittle Bros. $f$.

Wieck, Frederick G. $f$.

Wiegand \& Lorenz $f$.

Wildy, D. C. $n$.

Wiley, H. S. $n$

Wilken, V. F. $f$.

Willbeck, J. S. $n$.

Willburger, Richard M. $f$.

Williams, Ezra $n$.

Wilson, George W. $f$.

Wilson, Thomas C. $n$.

Wilson, William $n$

Wilson, William C. $n$.

Wilson, William C. $f$.

Windolf, John $f$.

Winkelmann, Louis $f$

Winters, Robert $f$

Wipperman, H. $n$

Wisse, David $n$.

Witten, Daniel $f$.
Milton, Ulster co

Spring Valley, Rockland co

23 Fulton St., New York city

I 8 Cortlandt St., New York city

Geneva, Ontario co

Middle Village, Queens co

343 East Ave., Rochester

362 Warren St., Brooklyn

Cor. Genesee and Noyes Sts., Utica

Union Ave., n'r E. 165th St., N. Y. city

Troy, Renssellaer co

Norton n. North Clinton, Rochester

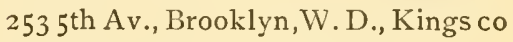

36 North Pearl St., Albany

Lansingburgh, Rensselaer co

Madalin, Dutchess co

2 I 2 North Ave., Rochester

Brooklyn, W. D., Kings co

Bay Ridge, Kings co

300 Lexington Ave., Albany

Jamestown, Chautauqua co

Baiting Hollow, Suffolk co

I 28 East I24th St., New York city

Skaneateles, Onondaga co

Henrietta, Monroe co

44 South Clinton St., Rochester

Rochester, Monroe co

28 North Pearl St., Albany

2053 3d Ave., and Ogden Ave. corner

West I 59th St., New York city

Long Island City, Queens co

Albany

Cayuga, Cayuga co

Corner 6th Ave. and West 129th St., New York city

Kinderhook, Columbia co

209 East 35th St., New York city

Rochester, Monroe co

8886 th Ave., New York city

Brighton, Monroe co

Geneva, Ontario co

Long Island City, Queens co

45 West 14th St., New York city

Manhatten, near Ioth Av., New York city

I6 5 West I 5 th St., New York city

Cortland, Cortland co

Brooklyn, Kings co

Mamaroneck, Westchester co

Hornellsville, Steuben co 
Wocker Bros.

Wood, Isaac C., \& Bro. $n$.

Wood, S. C. $n$

Wright, Thomas $n$

Yates \& Russell $n$

Yeomans, A. $n$.

Yeomans, T. G., \& Sons $n$.

Young \& Elliott $f$

Young, John $f$

Young, Thomas

Zahn, Charles $f$.

Zahn, Frederick $f$

Zahn, Otto $f$

Zeh, Philip $f$

Zimmerman, Godfrey \& Sons

Zundel, Charles $\mathrm{H}$. $f$.
Flatbush, Kings co

Rossville, Richmond co

Fishskill, Dutchess co

Knowlesville, Orleans co

Rochester, Monroe co

Rochester, Monroe co

Walworth, Wayne co

Walworth, Wayne co

I 8 Cortlandt St., New York city

Lansingburgh, Rensselaer co 26 West 28th St., New York city 284 Spring St., New York city 286 Grand St., New York city 390 Grand St., New York city Brooklyn, W. D., Kings co 571 Main St., Buffalo, Erie co Haverstraw, Rockland co

\section{NORTH CAROLINA.}

Anthony, G. $n$

Craft, N. Watson $n$

Crawford \& Bro. $n$

Denson, C. B. $n$.

Kestller, Chailes

Langenour, A. L.

Lindley, J. Van $n$

Merrell, J. M. $n$.

Osborn, J. R. $n$

Petar, Charles $n$.

Vandiver, J. W. $n$

Van Lindly, J. $n$.

Welborn, H. R. n

Westbrook, J. S., \& Bro. $n$.

Wilson, S. Otro, \& Co. $n$

- Greensboro, Guilford co
- Shore, Yadkin co
- Statesville, Iredell co
Pittsboro, Chatham co
- Statesville, Iredell co
- Footville, Yadkin co
- Greensboro, Guilford co
- Edneyville, Henderson co
- Bethany, Davidson co
- Ridgeway, Warren co
- Weaversville, Buncombe co
- Salem Junction, Guilford co
- High Point, Guilford co
- Faison, Duplin co
- Raleigh, Wake co

\section{OHIO.}

Ackerman, Jacob $n$. . . Newark, Licking co

Albaugh, N. H., \& Son $n$. . Tadmer, Montgomery co

Aldrich, A. $f$. . . Springfield, Clarke co

Anderson, Charles $f$. . Hillsboro, Highland co 
Arnold, Frederick $f$

Ashworth, W. \& J. n

Astry, William $n$.

Bachelder Bros. $f$

Bahls, John $f$

Barnes \& Bro. $f$.

Barnes, J. A. n

Barnes, L. F. $f$

Bashaw, L. H. $f$.

Batt, Mathias

Bauder, S. C. $n$.

Beaumont, W. H. $f$

Bechtel, Pierce $n$

Beckle, Anthony $f$

Beebe, G. A.

Bennett \& Johnson $f$.

Berlin, B. F. $n$.

Bernard, F. F. $n$.

Bertrand, Henry $f$

Betscher, George $n$

Black, R. G. $n$.

Blair, G. F. $n$

Bloomfield, E. C. $f$

Boeck, Robert $f$.

Bohland, John $f$.

Bohlender, Peter $n$

Bohls, John $f$.

Bolia, William $f$.

Bollman, William $f$

Boner, M. L. $n$.

Bonsall, J. E. $f$

Bonsal, Mark $n$

Bork, Philip $n$

Bowhall, H. n

Bowman \& Breckbill $n$

Breece, M. W. $n$.

Brown, B. H. $n$.

Brown, Charles $f$.

Brown, D. \& H. $n$

Brown, Thomas $n$

Buell \& Co. $f$

Burns, John $f$

Butz, Paul, \& Son $n$

Caldwell, Thomas

Call, S. W. n

Campbell, Archibald $f$.

Campbell, George IW. n

Campbell, Thomas N. $f$
8 Meyer Ave., Cleveland co

Tippecanoe City, Miami co

Franklin Square, Columbiana co

Detroit, opp. Lake Ave., Cleveland

1273 St. Clair Ave., Cleveland

Groesbeck, Hamilton co

Springfield, Clarke co

Centreburgh, Knox co

East Rochester, Columbiana co

Dayton, Montgomery co

Birmingham, Erie co

79 Euclid Ave., Cleveland

Troy, Miami co

Carthage Pike, Clifton, Cincinnati

Rocky River, Cuyahoga co

Hillsboro, Highland co

Louisville, Stark co

Painesville, Lake co

Rockdale Ave., Avondale, Cincinnati

Canal Dover, Tuscarawas co

Bremen, Fairfield co

Westerville, Franklin co

Middle Branch, Stark co

Red Bank Ave., Mt. Lookout, Cincinnati

59 Linden St., Cleveland

Tadmer, Montgomery co

Cleveland, Cuyahoga co

ro47 Colerain Ave., Cincinnati

Brooklyn Av., 25th Ward, Cincinnati

Bellville, Richmond co

Salem, Columbiana co

Salem, Columbiana co

Tiffin, Seneca co

Painesville, Lake co

Donnelsville, Clarke co

Delaware, Delaware co

Oxford, Butler co

Canton, Stark co

Brandt, Miami co

Medway, Clarke co

Springfield, Clarke co

I4 Danforth St., Cleveland

Springfield, Clarke co

Spring Grove Ave., 25th Ward, Cincinnati

Perry, Lake co

30i Cedar Ave., Cleveland

Delaware, Delaware co

3or Cedar Ave., Cleveland 
Carr, W. W., \& Co. $f$.

Carson, IV. $f$

"Champion Seed and Floral Co." $n$

Chapman, John

Chapman, W. $n$.

Charlesworth, David $f$.

Cincinnati Floral Co. $f$

Clemens, W. $n$.

Cole, J. J. $n$.

"Columbus Nurseries" $n$

Cook, C. M. \& J. S. $f$.

Cook, W. C. $f$.

Corwin, George $n$

Crawford, N. W., \& Son $n$.

Crawford Nursery $n$

Critchell, B. P., \& Co. $f$

Culp, Jacob $n$

Cunningham, William $f$

Cushion, Thomas $f$

Dana, George, \& Son $n$

Dant, Valentine $f$

Davis, Joseph $f$.

Day, John $n$

Day, Mrs. D. W. n

Dean, George W.

DeMuth, William M.

Dilger, William $f$.

Dowd, J. F. $f$.

Dwight, G. W. $n$.

Eadie, James $f$.

Eadie, Miss Jennie M.

Eck, John $f$.

Ely, W. B. $f$.

Engle, Louis $f$

English, L. G. $n$.

Erhardt, Henry $f$.

Evans, J. M. $n$.

Evans, Maurice $f$.

Evans, R. D. $n$.

Fassett, F. E., \& Bro. n

Faust, C. W.

Feldmann, Joseph $f$

Fergus, S. R. $n$.

Fisher, Anthony $f$

Fissel Bros. n

Flanagan, Patrick $f$

Flattery, W. S., \& Son $n$

Fogler \& Haines $n$

Fookes, A. L. $n$.
Yellow Springs, Greene co

Cadiz, Harrison co

Springfield, Clarke co

New Milford, Portage co

Glendale, Hamilton co

2 io Sibley, Cleveland

I87 West 4th St., Cincinnati

Station A, Cleveland

Lima, Allen co

Columbus, Franklin co

Myrtle Ave., IValnut Hill, Cincinnati

I3 West 4th St., Cincinnati

Mount Gilead, Morrow co

East Carmel, Columbiana co

Cuyahoga Falls, Summit co

I97 West 4th St., Cincinnati

Franklin Square, Columbiana co

Ludlow Ave., Clifton, Cincinnati

Wellington, Lorain co

Belpre, Washington co

Mad Anthony St., 25th Ward, [Cincinnati

Piqua, Miami co

Fremont, Sandusky co

Ravenna, Portage co

Kent, Portage co

Whitehouse, Lucas co

Sandusky, Erie co

I66 Woodward Ave., Cincinnati

Clyde, Sandusky co

Cleveland, Cuyahoga co

I05 Euclid Ave., Cleveland

I2 William St., Cleveland

Washington C. H., Fayette co

Canton, Stark co

Marysville, Union co

Ludlow Ave., Clifton, Cincinnati

Salem, Columbiana co

Columbus, Franklin co

Washington, Fayette co

Ashtabula, Ashtabula co

Canton, Stark co

$385 \% / 2$ Vine St., Cincinnati

Tadmer, Montgomery co

Central Ave., Clifton, Cincinnati

New Carlisle, Clarke co

Akron, Summit co

Wooster, Wayne co

Tiffin, Seneca co

Columbus, Franklin co 
Ford, Frank, \& Son $n$.

Forder, Alfred $f$.

Freeman, Mrs. A. D., \& Sons

Freeman, Isaac $n$

Freeman, Isaac $n$

French \& Andrews $n$.

Gairnes, David $f$.

Gardner, C. C. $f$.

Gardner, Jacob $n$.

Gardiner, W. $f$

Gardner \& Huntsman $f$

Garrett, S. H. $n$.

Gassand, Frederick $f$.

Gasser, J. M. $f$

Getz, Charles A. $f$

Gierspeck, Otto $f$.

Glanfield, George $f$

Glins, Henry $f$.

Glins, Joseph $f$

Goettle, Mrs. E. $f$

Graham, Alexander $f$.

Graham \& Johnston $n$.

Grant, Miss Ella L. $f$.

Gravett, John $n$

Green, J. L. $n$

Green, L., \& Son $n$

Greenler, J. S. $n$.

Greuninger, Paul

Grimes, William $n$

Guy, Albert $n$.

Guy, Hezekiah $n$.

Hale \& Son $n$

Hall, E. \& Son $n$.

Hall, James $f$.

Hall, Thomas P. $n$

Hampton, William C. $n$

Hannan, Dennis $f$

Hazelton, John $n$.

Heberling, H. G. n

Heike, Peter $f$

Heikes, Charles D. $n$.

Heikes, Isaac, \& Son $n$

Heikes Nursery Co. $n$.

Hemme, George

Henning, Christopher $f$

Herbert, Mrs. R. L. $f$.

Hine, D. A. $n$
Ravenna, Portage co

Linwood. Road, East Walnut Hills. Cincinnati

Tadmer, Montgomery co

Tippecanoe City, Miami co

New Carlisle, Clarke co

Rockport, Cuyahoga co

2883 Broadway, Cleveland

Freedom, Portage co

Orrville, Wayne co

I 99 West $4^{\text {th }}$ St., Cincinnati

I66 Walnut St., Cincinnati

Mansfield, Richland co

453 Scoville Ave., Cleveland

29 Euclid Ave., Cleveland

Harrison Pike, 24th Ward, Cincinnati

N. E. cor. Tod St. and Evergreen,

Cleveland

356 Sterling Ave., Cleveland

639 Vine St., Cincinnati

Spring Grove Ave., 25th Ward, Cincinnati

200 West 4th St., Cincinnati

Elyria, Lorain co

Reynoldsburg, Franklin co

277 Jennings Ave., Cleveland

Lancaster, Fairfield co

Granger, Medina co

Perry, Lake co

Defiance, Defiance co

Oak and Highland Aves., Cincinnati

Portsmouth, Scioto co

Franklin Square, Columbiana co

Franklin Square, Columbiana co

Mogadore, Summit co

Clyde, Sandusky co

Rush Run, Jefferson co

Barnesville, Belmont co

Mount Victory, Hardin co

Central Ave., Clifton, Cincinnati

Delaware, Delaware co

Short Creek, Harrison co

60 Holmden Ave., Cleveland

Tippecanoe City, Miami co

Tippecanoe City, Miami co

Dayton, Montgomery co

SIdney, Shelby co

Forest Ave., Avondale, Cincinnati

East Liverpool, Columbiana co

Akron, Summit co 
Hippard, E. $f$

Hoover \& Gaines $n$

Houston \& Leggett $n$.

Hoyt, Amasa $f$

Hughes, Robert $f$

Imley, William S. $n$

Israel, Jasper $n$

Jackson, Charles J. $f$.

Jackson, S. S. $f$.

Jayne \& Cole $n$

Jaynes, Harris $f$.

Jenkins, J. $f$.

Johnston, Thomas W. $f$

Jordan, J. B., \& Son $f$.

Juniet, Louis $f$.

Keen, Samuel $f$.

Kelly, Robert A. $f$

Kinsey, Samuel $f$

Kirchner, Thomas $f$

Knott, Thomas $f$.

Kresken, Mrs. H. A. $f$.

Kurtz, John $n$

Kyle, David M. $n$

Lamborn, Leroy $f$

Lee, D., \& Son $n$.

Lee, George W. $f$.

Lee, Joshua $f$

Leist, Jacob P. $n$.

Lewis, A. G. $f$

Lobingier, Quincy A. $f$

Lodders, John $f$

Lunn, James $f$

Lytle, R., Jr.

Maddocks, A. W. n

Magrie, George $f$.

Magrie, Matilda $f$

Maltoon, James $n$

Mastern, Henry $n$

Mauthey, Ernst $f$.

Messmore, E. $f$.

Miller, Mrs. E. $n$.

Miller, G. H., \& Son

Miller, George L. $n$

Milner, D. N. $f$

Milton, Mansfield \& Co. $f$.

Mooney, S. W. $n$.

Moore, Nathan, \& Sons $n$.

Moore, S. R. n

Morrisnn, J. n
Youngstown, Mahoning co

Dayton, Montgomery co

Lowellville, Mahoning co

Warren, Trumbull co

887 Euclid Ave., Cleveland

Zanesville, Muskingum co

Beverly, Washington co

52 Walnut St., Cincinnati

Cincinnati, Hamilton co

Painesville, Lake co

I 549 Euclid Ave., Cleveland

Winona, Columbiana co

Youngstown, Mahoning co

Mansfield, Richland co

New Baltimore Pike, Fairmount, Cincinnati

Franklin Square, Columbiana co I94 West 4th St., Cincinnati

Kinsey, Montgomery co

Garden, cor. Baden Ave., Cleveland

Mound St., Avondale, Cincinnati

305 Vine St., Cincinnati

Leipsic, Putnam co

Xenia, Greene co

Alliance, Stark co

Madison, Lake co

Homeworth, Columbiana co

Homeworth, Columbiana co

East Ringgold, Pickaway co

Youngstown, Mahoning co

Steubenville, Jefferson co

Hamilton, Butler co

I 390 Garden St., Cleveland

Williamsburg, Clermont co

Toledo, Lucas co

I80 Race St., Cincinnati

I 80 Race St., Cincinnati

Atwater, Portage co

Sandusky, Erie co

Ludlow Grove, Hamilton co

Minerva, Stark co

Ironton, Lawrence co

Norwich, Muskingum co

Stockton, Butler co

Mt. Pleasant, Jefferson co

Youngstown, Mahoning

Pleasant Ridge, Hamilton co

Sidney, Shelby co

Zanesville, Muskingum co

Cadiz, Harrison co 
Muchmore \& Son $n$ Mueller, Conrad $f$ Mulert, Hugo $f$.

Mumma, George R. n . Murdock, H. G. n Myers, H. H. n

McBeth, Thomas McCallum, M. H. $n$ McClelland, Miss C. $f$. McCullough, H. $f$ McFadden, F. T. $f$ McGregor Bros. $f$

McKellar, William $n$ McLane, Joseph V. $f$. McMullen, William $f$. McVeigh, G. W. n Neff, I. C. $n$

Nichols, Jesse $f$

Nichols, John $n$. Niles, George W.

Ohmer, M. $f$

Osborn, C. H. $f$

Paddock \&-Bros.

Palmer, F. R. $n$

Penney, George W. $n$.

Pentland, Francis $n$

Perdue, W. P. \& T. K. $f$

Perkins, L. A. $f$.

Peters, C. A. $f$.

Peters, D. E. $n$

Peters, George \& Son $n$

Pickett, G. S. $n$. .

Pierce, C. D., \& Bro. $n$

Pierce, L. B.

Pollard, Richard

Porter, S. P. $n$

Poste, John $f$

Probeck, George J. $f$.

Pumphrey, John S. $n$. Queen City Floral Bazar Rall, John n

Rathbone, W. W. n

Reed, W. W. \& J. A. n

Reeser, Charles A. $f$

Reeser, George F. $f$

Reigel \& Brehmer $n$

Ring, Daniel $f$

Ritter, Frederick W., Sr. $f$.
Fremont, Sandusky co

Grand Ave., 24th Ward, Cincinnati

507 Race St., Cincinnati

Dayton, Montgomery co

Farmdale P. O., Trumbull co

Canton, Stark co

Springfield, Clark co

Youngstown, Mahoning co

Columbus, Franklin co

Ellison Ave., Mt. Lookout, Cincinnati

Cincinnati, Hamilton co

Springfield, Clark co

Chillicothe, Ross co

Barnesville, Belmont co

Steubenville, Jefferson co

McConnellsville, Morgan co

Duncan's Falls, Muskingum co

7or Ansel Ave., Cleveland

Franklin Square, Columbiana co

Edgewood Road, East Walnut Hills, Cincinnati

Dayton, Montgomery co

Mt. Vernon, Knox co

Cleveland

Mansfield, Richland co

Newark, Licking co

Lockland, Hamilton co

New Martinsburg, Fayette co

Farmdale P. O., Trumbull co

Riverside, Cincinnati

Osborn, Green co

Troy, Miami co

Clyde, Sandusky co

Sparta, Morrow co

Tallmadge, Summit co

74I Woodland Ave., Cleveland

West Richfield, Summit co

Columbus, Franklin co

rozg St. Clair Ave., Cleveland

Celina, Mercer co

I95 West $4^{\text {th }}$ St., Cincinnati

Osborn, Green co

Marietta, Washington co

Canton, Stark co

Springfield, Clark co

Springfield, Clark co

Adelphi, Ross co

Hunt, north of Montgomery Road, Cincinnati

Dayton, Montgomery co 
Robinson, F. M. $n$

Robinson \& Moyer $n$

Romans, John B. $f$

Rose, Lucius $n$

Sacket, Ferson $f$.

Sager \& Bro. $n$

Schaeffer, Reinhold $f$.

Schepp, Canrad $f$

Schueren, C. Theodore, Sr. $f$

Sears, Benjamin $n$

Sears, George L. $f$

Seefried, John $f$

Seitner, Ernst $f$

Simmons, William P. \& Co. $n$

Simpkins, Ephraim $n$.

Simpson, John $f$.

Sked \& Co. $f$

Sked, Misses Jane J. \& M. A. $f$.

Sked, J. F., \& Co. $f$

Sked, W. J., \& Co. $f$

Smith, S. F. $x$

Smith, W. H., \& Son

Stanton, William $n$

Stockstill, James $f$

Sunderbruch, A., \& Son $f$

Sutts, Henry $n$

Swan, W. S. $n$

Swanger, A. J. $n$.

Templin, Louis $n$

Templin, Louis, \& Sons $n$.

The Storrs \& Harrison Co. $n$

Townsend, George $n$

Turley, P. L. $n$.

Tuttle, B. $n$.

Underwood, John

Untemacher, John F. $f$

Vandervoort, J. M. $n$

Vick, H. E. $f$

Vogle, George $f$.

Wagner, Charles R. $f$.

Wait, E. R.

Wallace, F. G. $f$.

Walz, A., \& Son $f$

Webb, L. B. $n$

Weigel, Christopher $f$.

Weltz, Leo $n$

Whitacre, T. L. $n$

White, Birney H. $f$

Whitney, J. D., \& Bro. $n$
Amelia, Clermont co

Findlay, Hancock co

Columbus, Franklin co

Akron, Summit co

Lewis Centre, Delaware co

North Bristol, Trumbull co

Denham St., n. Fairmont, Cincinnati

Mad Anthony St., 25th Ward, Cin-

cinnati

703 Superior Ave., Cleveland

Bucyrus, Crawford co

Elyria, Lorain co

Carthage Pike, Clifton, Cincinnati

Dayton, Montgomery co

Geneva, Ashtabula co

Belfast, Claremont co

Minerva, Stark co

Cleveland, Cuyahoga co

I2 Marion Ave., Cleveland

Westerville, Franklin co

I 45 I St. Clair Ave., Cleveland

Marietta, Washington co

New Carlisle, Clarke co

Barnesville, Belmont co

Dayton, Montgomery co

$2001 / 2$ West $4^{\text {th }}$ St., Cincinnati

Youngstown, Mahoning co

Cadwallader P. O., Tuscarawas co

Shiloh, Richland co

Loveland Station, Mahoning co

Greenford, Mahoning co

Painesville, Lake co

Gordon, Darke co

Proctorville, Lawrence co

Clyde, Sandusky co

Columbus

Newark, Licking co

New Antioch, Clinton

Alliance, Stark co

Hubbard, Trumbull co

Portsmouth, Scioto co

Ravenna, Portage co

Columbus, Franklin co

Boyd St., 25th Ward, Cincinnati

Salem, Columbiana co

Cleveland, Cuyahoga co

Wilmington, Clinton co

East Rochester, Columbiana

Salem, Columbiana co

Norwalk, Huron co 
Whitney, J. V., \& Son $n$ Wieland, William $f$ Wilhelmy, Mathias A. $f$ Wilson, Mrs. E. $f$ Wood, H. S. $n$ Woodall, Thomas Woodruff, H. M. n Young, J. F. $f$. Young, James H. n
Montville, Geauga co

- Harvey Ave., Avondale, Cincinnati 9 Meyer Ave., Cleveland

- Barnesville, Belmont co

Marysville, Union co

Chestnut St., Walnut Hills, Cincinnati

Geneva, Ashtabula co

Dayton, Montgomery co

Troy, Miami co

\section{OREGON.}

Burkhardt Bros. $f$

Carter, Henry $n$.

Dickinson, O. $n$

Gangloff, A. $n$

Hansen, H. $f$

Lambert, J. H. $n$.

Morgan, C. F. $f$.

"Portland Nursery Co." $n$

Prettyman, William H. $f$.

Settlemire, J. H. n

Settlemires, H. $n$.

Walling, G. W., \& Son $n$
East Portland, Multnomah co

Whiteaker, Marion co

Salem, Marion co

LaGrande, Union co

Portland, Multnomah co

- Milwaukee, Clackamas co

Sellwood, Multnomah co

82 Morrison St., Portland

East Portland, Multnomah co

Woodburn, Marion co

- Tangent, Lynn co

- Oswego, Clackamas co

\section{PENNSYLVANIA.}

Achelis, George $n$. . West Chester, Chester co

Adams, A. S. $n$. . . . Red Stone, Fayette co

Aderhold, H. H. $n$. . . Saxonburg, Butler co

Ahlers, Louis $f$. . . . Station I, Roxborough, Philadelphia

Ahern, Patrick $n$. . . . 407 North 36 th St., Philadelphia

Akers, Alvah $f$. . . Johnstown, Cambria co

Albert, Theo. $f$. . . . Reading, Berks co

Albright, John $f$. . . . Pencoyd, Montgomery co

Alburger \& Bro. $n$. . . Ridge Ave. and Huntingdon, Phila.

Alburger, Henry $f$. . . Gray's Lane n. Woodland Av., Phila.

Ailgaier, Christian $f$. . . 22d and Clearfield, Philadelphia

Allgaier, Frederick (Estate of) $f$. Passyunk Road and Broad, Phila.

Allis, S. J. $n$. . . . . Erie, Erie co

Altorfer, Gerald $n$. . . West Chester, Chester co 
Alvord, N. H. $f$. Amidon, C. F. $n$. Anderson, George $f$ "Antietam Nursery" $n$ Archer, James $f$.

Armstrong, A. R. $n$ Ashford, John E. $n$ Auckland, John $f$ Bachler, Sophia $f$ Bader, John $f$ Bailey, W. J. $f$ Baldeston, J. E. Ball, Charles D. $f$ Ball, Philip R. $f$. Banyard, E., \& Son $f$. Barker, Basil $f$. Barrows, James $f$. Barry, Thomas E. $f$ Bascom, F. A. Bauman, George $f$ Baumann, Louis C. $f$ Bearn, David $f$ Beck, J. G. $n$ Beck, John H. $f$ Beckert Bros Beckert, Jacob Beckert, W. C. $f$. Bell, Conrad H. $f$ Bell, W. T. $f$

Bender, George J. $f$ Bennett, Wm., \& Son Berst, Titus

Biddle, F. C. Billger, Louis $f$ Bissett, Mrs. Mary $f$ Bitner, Henry $n$. Bogan, John $f$

Bogan, Michael $f$. Bolton, Wilmer P. $n$ Bortmas, J. WV. Boyd, William $f$ Boyer, Gottlieb $f$. Breining, Elwood T. Brenneman, I. D. $f$ Brenneman, William H. $f$. Brewster, William n Bridgland, George T., \& Sons Brierly, William B. $n$. Brings, Girard $f$. Brinton, Williain P. $n$.
Towanda, Bradford co

Rundels, Crawford co Station B, Paschallville, Phila. Waynesboro, Franklin co 52d and Pear Sts., Philadelphia West Springfield, Erie co North Wales, Montgomery co Station F., Frankford, Philadelphia Lancaster, Lancaster co Allegheny, Allegheny co Harrisburg, Dauphin co New Hope, Bucks co Station M, Holmesburg, Philadelphia Reading, Berks co zoth and Ridge Ave., Philadelphia Allegheny, Allegheny co Station G, Germantown, Phila. General Wayne, Delaware co Greenville, Mercer co Station I, Roxborough, Philadelphia Station G, Germantown, Phila. 33 I Wallace St., Philadelphia Yorkana, York co 904 N. 5th St., Philadelphia Allegheny, Allegheny co Allegheny, Allegheny co Allegheny, Allegheny co I 108 Girard Ave., Philadelphia

Franklin, Venango co Harrisburg, Dauphin co Carrick, Allegheny co Erie, Erie co Chadd's Ford, Delaware co Station H, Mount Airy, Philadelphia 333 Tasker St., Philadelphia Bigler P. O., Adams co Station B, Paschalville, Philadelphia 2 Ist and Columbia Ave., Philadelphia Liberty Square, Lancaster co Butler, Butler co 9I2 Montgomery Ave., Philadelphia I oI 7 North gth St., Reading, Berks co 5012 Westminster Ave., Philadelphia Harrisburg, Dauphin co Harrisburg, Dauphin co Erie, Erie co

Williamsport, Lycoming co Williamsport, Lycoming co K St., near Venango St., Phila. Christiana, Lancaster co 
Brooks, Eli n

Brown, Catharine $f$

Brunner, Peter $f$.

Buist, Robert, Jr. n

Burpee, W. Atlee, \& Co. n .

Butz, Paul, \& Sons $f$

Cadwallader \& Bro. $f$.

Caird Bros.

Cameron, H. L. $f$

Campbell, George $n$

Campbell, James H., \& Sons

Campbell, Joseph H. $f$

Carpenter, George T. $f$

Carr Bros. $n$.

Carr, L. W. $n$

Cassel, Jacob E. n

Caughey, John M. $f$

Cavanaugh, Thomas $f$.

Chaapel, Harry $\dot{f}$.

Chambersburg Nursery $n$

Chase, R. G., \& Co. $n$.

Chatterton, Jabez $f$.

Claphanson, Samuel $f$.

Clark, George R., \& Co. $f$.

Clark, Michael $n$.

Clark, Robert, Jr. $f$.

Claude, John $f$.

Cliffe, David $f$.

Clifford, N. B. $n$.

Cioor, E. F. $n$

Cloud, William P.

Clous \& Son

Cole \& Shelley $f$.

Coleman, E. V. $n$

Colflesh, John W.

Colvin, George H.

Connelly, John J.

Conrad Bros. $f$

Conway, John B. $f$

Cooper, Calvin $n$.

Cope Bros. $f$.

Copper, John

Corl, Miles $n$

Cornell \& Michler $f$

Craig \& Bro. $f$

Crall, I. S. $f$.

Craven, Jacob $n$

Crawford, Charles $f$

Crawford, Robert, Jr.
"Centre Road" Station, Crawford co I 232 North 26th St., Philadelphia

Coopersburg, Lehigh co

922 and 924 Market St., Philadelphia

475 North 5 th St., Philadelphia

New Castle, Lawrence co

Newtown, Bucks co

Elizabeth, Allegheny co

Titusville, Crawford co

Athens, Bradford co

N. W. cor. 8th and Sansom Sts., Phila.

Station G, Rising Sun, Philadelphia

55th St. and Wyalusing Ave., Phila.

Erie, Erie co

Erie, Erie co

Mount Joy, Lancaster co

Pittsburg, Allegheny co

Church Lane and Greenway Ave.,

Philadelphia

Williamsport, Lycoming co

Chambersburg, Franklin co

30 South Merrick St., Philadelphia

Station F, Frankford, Philadelphia

25 Goodman St., Philadelphia

Scranton, Lackawanna co

West Chester, Chester co

502 Washington Ave., Philadelphia

Fernwood, Delaware co

Station G, Germantown, Phila.

Holicong, Bucks co

Claysburg, Blair co

Kennett Square, Chester co

Reading, Berks co

55th and Walnut Sts., Philadelphia

Centretown, Mercer co

Station B, Paschalville, Philadelphia

Dalton, Lackawanna co

Bryn Mawr, Montgomery co

McKeesport, Allegheny co

Phœnixville, Chester co

Bird-in-Hand, Lancaster co

Hatboro, Montgomery co

Newtown, Bucks co

Harrisburg, Dauphin co

Easton, Northampton co

203 S. I Ith St., and $49^{\text {th }}$ and Market

Sts., Philadelphia

Monongahela City, Washington co

York, York co

$55^{\text {th }}$ and Woodland Ave., Phila

227 South I Ith St., Philadelphia 
Crossley, Robert $n$

Crouch, A: E. $n$.

Cunningham, George W'. n

Cunningham, John $f$.

Curtin, Daniel $f$.

Curven, George F. $f$.

Darlington Bros. $n$

Darlington, E. D. $f$

Dennison Bros. $f$.

Delahunt, Edward $f$

Devereaux, H. M. $n$.

DeWitt Bros. $f$.

Dick, John, Jr. $f$.

Dickey, Samuel $f$.

Didden, William $f$

Dietrick, Edward H. $f$.

Dillon, J. L. $f$.

Dinger \& Conard Co. $n$

Donaldson, James $f$

Dreer, Henry A. $f$

Dressler \& Son $f$.

Dunn, Clarence A. $f$

Dunn, Michael C. $f$.

Dwight, F. G. $f$

Eagle, Louis J. $f$.

Easton, IV. R.

Egee, Robert, \& Son $f$.

Eisele, Christian $f$

Eisele, John G. $f$.

Eldridge, William M. $f$

Elliott, Benjamin A. n

Elsworth, A. B.

Emerick, A. $f$

Engle \& Bro. $n$.

Engle, H. M., \& Son $n$

Ensinger, G. IV. $f$

"Enterprise Green Houses

Eschbach, Charles $f$

Evans, Charles F.

Evenden Bros. $n$.

Everitt, J. A., \& Co. $u$.

Fagley, Frederick $f$

Falck, M., \& Sons $f$

Farson, Daniel D. L. $f$

Faust \& Crawford $f$

Faust, William F., \& Son $f$.

Felton, O. B. M.

Fergus Bros. ,

Fergusson, David, \& Sons

Fesher, Philip $f$.
Mansfield, Tioga co

West Springfield, Erie co

Newtown, Bucks co

Norristown, Montgomery co

Station G, Nicetown, Philadelphia

Villanova, Delaware co

West Chester, Chester co

Doylestown, Bucks co

Belmont and City Aves., Phila.

Chester, Delaware co

West Springfield, 'Erie co

Bristol, Bucks co

53d and Woodland Ave., Phila.

West Grove, Chester co

Station B, Paschalville, Philadelphia

Wilkesbarre, Luzerne co

Bloomsburg, Columbia co

IVest Grove, Chester co

Kittanning, Armstrong co

7 I4 Chestnut St., Philadelphia

Station G, Tioga, Philadelphia

204 South 4 oth St., Philadelphia

61 2 Chestnut St., Philadelphia

Reading, Berks co

Belmont, Philadelphia

Corry, Erie co

Ioog Marshall St., Philadelp'

I 500 North I Ith St., Philar phia

I 332 North I 2 th St., Philauelphia

IVilkesbarre, Luzerne co

littsburg, Allegheny co

Allentown, Lehigh co

Bethlehem, Northampton co

Marietta, Lancaster co

Marietta, Lancaster co

Elm Station, P. R. R., Philadelphia

Germantown, Philadelphia

Lancaster, Lancaster co

Station F, Frankford, Philadelphia

Williamsport, Lycoming co

Watsontown, Northumberland co

I6I7 North Igth St., Philadelphia

Allegheny, Allegheny co

5oth and Vestminster Ave., Phila.

I04 South I6th St., Philadelphia

1430 Columbia Ave., l'hiladelphia

330 North $63 d$ St., Philadelphia

iVest Chester, Chester co

Ridge and Lehigh Ave., Phila.

Chambersburg, Franklin co 
Firestone \& Bro. $n$

Fitzpatrick \& Rue $f$

Fletcher, Israel $f$.

Flick, Thomas L. $f$

Floral Exchange $f$

Fox, Charles $f$

Fries, Jacob A. $f$.

Gaehring, Jacob F., Jr. $f$

Gaehring, Mrs. Mary M. $f$.

Garrow, H. S. $f$.

Gesner, Washington $f$.

Getz, C. A. $f$

Good, Daniel $n$

Good, E. B. $n$

Goodell, F. C. $n$

Graham, Hugh, \& Co.

Graham, Robert

Grassie, William

Gregg, Aaron $n$

Gregory, Joseph D. $f$.

Grieserver, J. B. $f$

Griffin \& Bro. $f$

Grigg, Charles H., \& Co. $f$.

Haas, Anton $f$

Haas, G. W. $f$

Habermehl, J. Jacob, \& Son

Hahman, Frederick $f$.

Haines, Henry C. $f$

Haines, Robert B. $n$

Hammers, A. M. $n$

Hanawalt, John S. $n$.

Hanson, William H. $f$.

Harding, James, Sr. $n$.

Harris, William K. $f$.

Harry, Winfield S. $f$

Haubert, John $f$.

Hauser, Theodore $f$

Heinz, Charles $f$.

Hellborn, Watson $f$

Hendrick, Thomas, \& Son $f$

Hensel, George O. $f$

Hepler, John C. $f$

Hess, Adam $n$.

Hess, Samuel R. n

Hiller, Casper, \& Son $n$

Himmelberger, W. W. $n$

Hofmeyer \& Neff $f$

Hoopes Bro. \& Thomas $n$.

Hornbrook, E. G., \& Co. $n$.

Hort, Herman $f$.
Latimore, Adams co

1424 Thompson St., Philadelphia

Station F, Bridesburg, Philadelphia

502 Callowhill St., Philadelphia

6 I 2 Chestnut St., Philadelphia

I 408 North Broad St., Phila.

Bethlehem, Northampton co

I807 Frankford Ave., Phila.

I 809 Frankford Ave., Phila.

Pittsburg, Allegheny co

67th and Greenway Ave., Phila.

Lancaster, Lancaster co

Manchester, York co

Manchester, York co

Indiana, Indiana co

I 8 th and Thompson Sts., ${ }_{2}$ Phila.

Harrisburg, Dauphin co

Station G; Germantown, Phila.

Carmichaels, Greene co

I642 South 2d St., Philadelphia

Douglassville, Berks co

Station F, Frankford, Phila.

1206 Chestnut St., Philadelphia

Dushore, Sullivan co

Meadville, Crawford co

2 I 19 West Park Ave., Philadelphia

Harrowgate Lane, near Front, Phila.

Station G, Germantown, Phila.

Cheltenham, Montgomery co

Indiana, Indiana co

McVeytown, Mifflin co

Elm Station, Penn'a R. R.

Montoursville, Lycoming co

Station B, Paschalville, Phila.

Conshohocken, Montgomery co

Cor. Ford Road and Belmont, Phila.

Reading, Berks co

Sharon, Mercer co

Newtown, Bucks co

Station G, Germantown, Phila.

Lancaster, Lancaster co

Reading, Berks co

Boalsburg, Centre co

Ephrata, Lancaster co

Conestoga, Lancaster co

Robesonia, Berks co

Allegheny, Allegheny co

West Chester, Chester co

Berwyn, Chester co

Station F, Frankford, Phila. 
Hoskins \& Giles $f$
Hudson, J. M. $f \cdot$
Hughes, George J. $f$
Hunderlpfund, Frederic
Iehle, Christian $f \cdot$
Iehle, Ernst G. $f \cdot$
Irving \& Valentine $f$
James, Harvey Q. $f$
Janney, Horace $n$
Jasper \& Shuster $f$
Jennings, C. W. $f$
Johnston, James $f$
Johnson \& Stokes $n$
Johnson, William $f$
Johnson, William S. $f$
Joyce Bros. $f$
Joyce, John D. $f \cdot$
Keech, J. F. $n$
Keeler, H., \& Son $f$
Keim, Jonathan $n$
Kemp, John $f$

Kemp, John G. $f$.

Kemp, H. H. $n$.

Kent, James, Jr. $n$

Kift, Joseph $n$

Kift, Joseph, \& Son $f$

King, Mrs. Henry, Sr. $f$

Kinnier, John $f$

Kirchner, Conrad

Kise, Samuel $f$

Koehler, Julius $f$.

Koessling, Charles $f$

Krause, Levin J. $f$

Krebs, Frederick S. $f$.

Kremer, D. W. $n$.

Kruger, A.

Kuser, P. \& J. $n$

Lake, Harmon $f$

Lake Shore Nursery $n$.

Landreth, David, \& Son $n$.

Langsdorf, Charles $n$.

Lanzford, John $f$. .

Latshaw, D. B. $n$

La Roche \& Stahl $f$

Laughlin, George $f$

Laughlin, G. A. $f$.

Layer, Isaac $f$.

Lazenby, George, \& Son $f$.

Le Gierse, Ferd. J. $f$
Reading, Berks co

I 404 Walnut St., Philadelphia

Downingtown, Chester co

Ioo3 Noble St., Philadelphia

Station T, Olney, Philadelphia

I 59 Sellers St., Frankford, Phila.

507 South I Ith St., Philadelphia

Altoona, Blair co

Newtown, Bucks co

175 Fountain St., Manayunk, Phila.

Station F, Olney, Philadelphia

Darby, Delaware co

I I 4 Market St., Philadelphia

Easton, Northampton co

Greenville, Mercer co

I 28 South I Ith St., Philadelphia

Pottsville, Schuylkill co

Oxford, Chester co

Altoona, Blair co

Pottstown, Montgomery co

Cor. Adams and Orthodox Streets,

Frankford, Philadelphia

Station F, Frankford, Phila.

Uniontown, Fayette co

37 I 4 Market St., Philadelphia

West Chester, Chester co

I 72 I 1/2 Chestnut St., Philadelphia

Norristown, Montgomery co

Station G, Germantown, Phila.

2Ist and Allegheny Ave., Phila.

Safe Harbor, Lancaster co

Station F, Frankford, Philadelphia

Meadville, Crawford co

Bethlehem, Northampton co

543 Belgrade St., Philadelphia

Pott's Grove, Northumberland co

Meadville, Crawford co

Boyertown, Berks co

2614 Ridge Ave., Philadelphia

Erie, Erie co

23 South 6th St., Philadelphia

Bendersville, Adams co

I I South I $3^{\text {th }}$ St., Philadelphia

Vincent, Chester co

I 237 Chestnut St., Philadelphia

Station G, Germantown, Phila.

I 8 I 4 Frankford Ave., Philadelphia

23d and Long Lane, Philadelphia

4309 Ludlow St., Philadelphia

4643 Lancaster Ave., Philadelphia 
Lease, Frederick $f$

Ledward, Matthew $f$.

Ledwards, J. J. $f$.

Lennox, Charles $f$

Lessig, Theo. $f$.

Lewisburgh Greenhouse $f$.

Line, L. A. $f$

Lobman, John $f$.

Long, Charles $f$.

Longsdorf, J. M. $n$

Longsdorff, C. L. $n$

Lonsdale \& Burton $f$.

Losch, John $x$

Louis, John $f$

Lucking, Mrs. Kate E. $f$

Ludwig \& Richter

Luitwieler, J. C. $n$

Lutz, August $f$.

Lyte, Lewis C. $n$.

Maddock, John $f$.

Nagee, John $f$.

Magill, James $f$.

Maginley, Thomas J. $f$

Martin, Isaac $n$

Mason, Edward $f$

Maule, William Henry $n$

Mearig, Isaac $f$.

Mecky, Charles $f$.

Meehan, Thomas n

Meehan, William E. $f$.

Mellott, Uriah $n$.

Mertz, G. Emanuel $f$.

Miller, G. $f$.

Miller, Frederick $f$

Miller \& Yates $f$.

Mish, D. H. $f$

Mood, T. S. $n$

Moon, Samuel C. $n$

Moon, William H. $n$

Moore, William T. $f$

Motsch, Louis $f$.

Mulberry, John $n$.

Muller, Karl $f$.

Muller, Karl, Jr. $f$

Murdoch, John R. \& A. $n$

Murdock, William \& James

Myers, Allen S. $f$.

McAdams, John $n$

McClane, James $f$

McDonald, David $f$
West Bridgewater P. O., Beaver co McKeesport, Allegheny co

Chester, Delaware co

E St. and Kensington Ave., Phila.

Station F, Frankford, Phila.

Lewisburg, Union co

Carlisle, Cumberland co

Harrisburg, Dauphin co

Station F, Frankford, Phila.

Mechanicsburg, Cumberland co

Flora Dale, Adams co

Chestnut Hill, Philadelphia

Allentown, Lehigh co

829 North Igth St., Philadelphia

626 North 5th St., Philadelphia

24 Federal St., Allegheny City

York, York co

3 I 2 North 3 Ist St., Philadelphia

Bird-in-Hand, Lancaster co

Turtle Creek, Allegheny co

I 40 North I 9 th St., Philadelphia

3100 North Broad St., Philadelphia

1335 North Ioth St., Philadelphia

Marlborough, Chester co

Frankford, Philadelphia

I 29 South Front St., Philadelphia

Leacock, Lancaster co

2720 Kensington Ave., Phila.

Germantown, Philadelphia

Germantown, Philadelphia

Ray's Hill, Bedford co

39 Buckius St., Philadelphia

Kimberton, Chester co

Paschalville, Philadelphia

Mt. Airy, Philadelphia

Lebanon, Lebanon co

Point Pleasant, Bucks co

Morrisville, Bucks co

Morrisville, Bucks co

Allegheny City, Allegheny co

Chestnut Hill, Philadelphia

Chatham, Chester co

2 I9 South 8th St., Philadelphia

67 th and Elmwood Ave., Phila.

I 2 Smithfield St., Pittsburg

Pittsburg, Allegheny co

Altoona, Blair co

Mt. Pleasant, Westmoreland co

50 I Emily St., Philadelphia

1419 Dauphin St., Philadelphia 
Sulvegf. F.E. 49 Proulst. Mouleast 
Rickman, Henry $f$.

Ricksecker, Edmund $n$

Rimby, Horace $f$.

Ritchie, Archibald, Jr. $f$

Ritchie, James $f$.

Roberts, J. A. $n$.

Robertson, Thomas

Rogers, Miss Mary $f$

Rohrer, A. D., \& Bro.

"Rowlandville Nursery"

Rumel, Mrs. T. $f$.

Rupp, H.S $f$.

Saunders, Joshua $f$

Savage, John $f$

Scannell, Michael

Schaeffer, William L. $f$

Schmidt, Joseph $f$

Schmitz, Charles $f$

Schroyer, George W.

Schuck, Charles $f$

Scott, Robert, \& Son $f$

Seal, Thomas F. n

Seamen, W. H. $n$

See, W. W.

Seidel, D. B.

Sheafer, Henry C. $f$

Shearer, Solomon "

Sheetz, Peter E. /

Shehey, J. $f$.

Shellem, John A. $f$

Shimer, A. P. $f$

Siebert, Charles T.

Sloan, Mrs. M. W.

Smiley Bros.

Smith, J. S. $n$

Smith, J. Y. $f$

Smith, William H. n

Snyder, Eckard $f$

Snyder, Solomon 1

Speaker, Charles

Speakman, E. B.

Starr, Charles $\mathrm{T}$.

Steckler, D. H. $f$

Stein, L. N. $f$

Steinbach, H. "n

Stephinson, J. G.

Sterling, J., \& Sons

Sterrett, S. WV.

Stetzer, Henry C. $f$

Stewart, William J.
Shepherdstown, Cumberland co

Nazareth, Northampton co

Collegeville, Montgomery co

5 I Goodman St., Philadelphia

1600 American St., Philadelphia

Malvern, Chester co

2223 Fairmount Ave., Philadelphia

Paschalville, Philadelphia

Lancaster, Lancaster co

Station F, Frankford, Philadelphia

333 Tasker St., Philadelphia

Shiremanstown, Cumberland co

Roxborough, Philadelphia

4219 Brown St., Philadelphia

2829 Amber St., Philadelphia

535 North 8th St., Philadelphia

Harrisburg, Dauphin co

3903 Ridge Ave., Philadelphia

Lancaster, Lancaster co

2455 North 8th St., Philadelphia

755 South Igth St., Philadelphia

Unionville, Chester co

Leetsdale, Allegheny co

Bradford, McKean co

Pottsville, Schuylkill co

I 808 North 24th St., Philadelphia

Tuckerton, Berks co

1200 Spring Garden St., Phila.

Norristown, Montgomery co

17 th and Tasker Sts., Phila.

Phœnixville, Chester co

Stanton Ave., E. E., Pittsburg

Marple, Delaware co

Pottsville, Schuylkill co

Mt. Lebanon, Allegheny co

Doylestown, Bucks co

Ior 8 Market St., Philadelphia

Allegheny City, Allegheny co

Reading, Berks co

Chestnut Hill, Philadelphia

Avondale, Chester co

Avondale, Chester co

Reading, Berks co

Harrisburg, Dauphin co

Everett, Bedford co

Girard, Erie co

Masontown, Fayette co

Carlisle, Cumberland co

Erie, Erie co

1518 Chestnut St., Philadelphia 
Stigale, Mrs. E. M. $f$. Stines, William $f$. Straucer, Reuben $n$ Strautz, August $f$. Strehle, Max $f$. Stuetzer, Ernest $f$ Supplee, P. Z. $n$. Sutherland, William $f$. Sutton, H. S. $n$ Sutton, James H. $f$ Swayne, William $f$ Taylor, Harry $f$. Thomas, Joseph W., \& Son. $n$ Tillinghast, Isaac F. $n$ Topley \& Winfield $f$. Trimble, Miss H. B. $n$. Trumbauer, Charles Q. $f$ Uber, George W. $f$ Ulrich, Frank $f$. Wagner \& Davis $n$ Wagner, W. H. $f$. Walker, E. S. $n$. Walker, Wilson C. $f$ Walter, Mrs. S. A. $f$ Walter, William Walterer, H. n

Waring, Edmund $n$ Waters, O. J. $f$

Weaver, Reuben $n$ Weiss, Eugene $f$. Weiss, Thomas $f$. Wentzell, Jesse B. $n$ Westcott, William H, $f$ Weissen, J. C. $n$. White, A. J. $n$ White \& Bro. $f$ White, Thomas W. $f$. Whitney, N. n Whitning, W. H. n Wickersham, Robert $n$ Wideman, Leroy $\mathrm{H}$. $n$ Williams \& Bro. $f$ Williamson, William Wilson, James B. $n$ Wilson, S. n Windolph, Joseph $n$ Wismer \& Lear $n$ Woeckner, Caroline $f$. Wolff, Julius $f$. Woltemate, Henry C. $f$
$55^{8}$ North $5^{\text {th }}$ St., Philadelphia

Rising Sun, Philadelphia

Nekoda, Perry co

\section{Lancaster, Lancaster co}

Cor. Erie Ave. and L St., Phila. 2148 North 6th St., Philadelphia Spread Eagle, Chester co 65th and Elmwood Ave., Phila Franklin, Venango co Honesdale, Wayne co Kennett Square, Chester co Harrowgate, Philadelphia King-of-Prussia, Montgomery co La Plume, Lackawanna co 4725 Pulaski Av., Germantown, Phil West Chester, Chester co Frankford, Philadelphia Paschalville, Philadelphia 925 Montgomery Ave., Philadelphia Landisburg, Perry co Tioga, Philadeiphia Camp Hill, Cumberland co 1229 North 52d St., Philadelphia Doylestown, Bucks co 2904 Ridge Ave., Philadelphia 3809 Powelton Ave., Philadelphia Tyrone, Blair co Jumonville, Fayette co Mastersonville, Lancaster co 63 N. 8th St., Philadelphia Germantown, Philadelphia Near Reading, Berks co 3443 N. $2 d$ St., Philadelphia York, York co Mill Creek, Huntingdon co 3135 Chestnut St., Philadelphia 231 North 8th St., Philadelphia East Charleston, Tioga co Wellsboro, Tioga co Bendersville, Adams co Curwensville, Clearfield co Sharon, Mercer co 2362 Lewis St., Philadelphia Washington, Washington co Mechanicsville, Bucks co Marietta, Lancaster co Wismer, Bucks co Erie, Erie co 34th and Ridge Ave., Philadelphia Germantown, Philadelphia 
Woltemate, Mrs. Rosalie Woods, D. R., \& Co.

Woods, T. A. $f$

Wright, G. I. $n$

Wright, John D. $n$

Wyatt, E., \& Son $f$

Wyatt, W. H. $f$

Young, Henry $f$

Young, William J. $f$

Youngken, Josiah G. $n$

Zamplaugh, James $f$.

Zimmermann, John $f$.

Zugg, Andrew $n$.
Germantown, Philadelphia

New Brighton, Beaver

Harrisburg, Dauphin co

Meadville, Crawford co

Curwinsville, Clearfield co

Tioga, Philadelphia

236 North I 3 th St., Philadelphia

Lancaster, Lancaster co

Germantown, Philadelphia

Richlandtown, Bucks co

I 58 Passyunk Ave. Market, Phila.

Lancaster, Lancaster co

East Petersburg, Lancaster co

\section{RHODE ISLAND.}

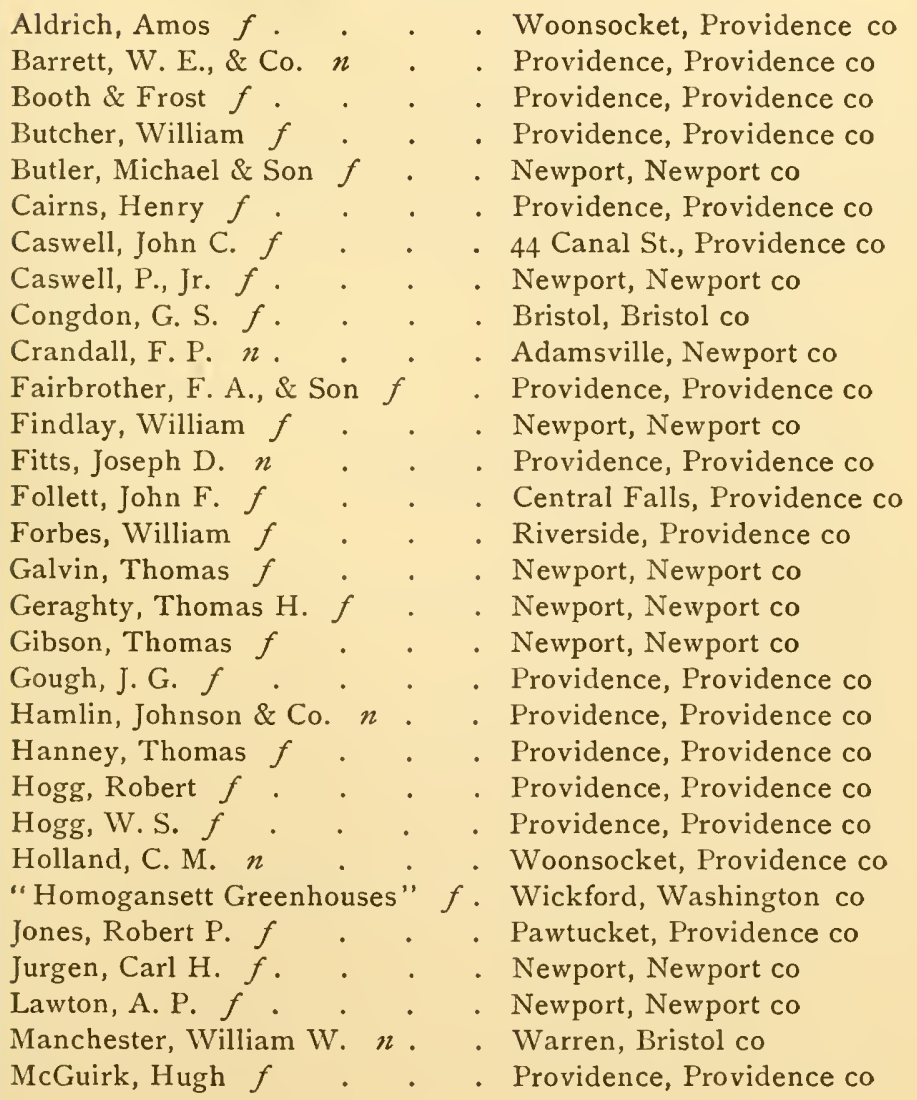


Nisbit, James $f$

Niven, Robert $f$

O'Connor, Timothy $f$.

O'Reilly, Frank $f$

Pierce, N. D., Jr., \& Co.

Potter, T. G. \& R. T. n

Sherman, G. L. $f$.

Smith, F. M. $f$.

Smith, William L. $f$

Thurston, Benjamin M.

Watson, William A. $n$.

Wilcox, Moses $f$.

Wright, Charles $f$
- Pawtucket, Providence co Providence, Providence co Providence, Providence co Rawtucket, Providence co Norwood, Kent co Rumford, Providence co Providence, Providence co Newport, Newport co Olneyville, Providence co Newport, Newport co Peacedale, Washington co Pascoag, Providence co - Cor. Franklin and Garden Sts., Providence

\section{SOUTH CAROLINA.}

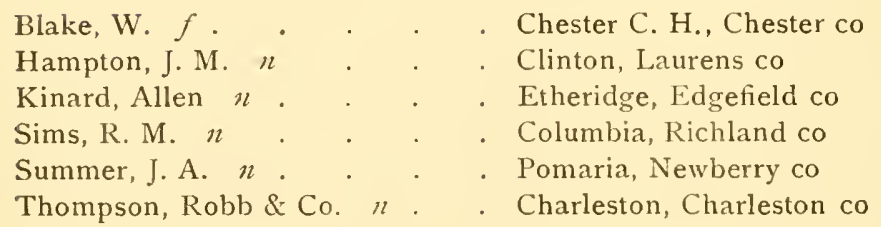

\section{TENNESSEE.}

Bailey, Mary E. $f$ Baker, A. \& S. L. " Biel, John, \& Son n Britton, William Capell, W. H. $n$ Cooper, J. A n Corney, J. B. $f$ Crusman, J. J. $f$ Currey, George $\mathrm{II}$. Deaderick, E. L. $n$ Ferrell, C. H. " Gartland, Thomas ( . . , Green \& Fentress $x$ Grizzard, IV. B. n Lanham, Harry
Chattanooga, Hamilton co

Madison, Davidson co

McKenzie, Carroll co

Estill Springs, Franklin co

Newbern, Dyer co

Nashville, Davidson co

Nashville, Davidson co

Clarksville, Montgomery co

Nashville, Davidson co

Jonesboro, WVashington co

Humboldt, Gibson co

Nashville, Davidson co

Coopertown, Robertson co

Huntingdon, Carroll co

$5^{\text {th }}$ and Chelsea, Memphis, Shelby co 
Nixon, E. S., \& Son
Nixon, H. M. $n$. Parker, John W. $n$

Rainey, Horace $n$ Smith, William Henry $x$ Spicer, R. A. $n$ Stark, John $n$. Stewart, James $n$. Stillwell, Charles A. $n$. Transon \& Co. $n$. Truett, Alphus $n$. Ullathorne \& Co. n Underhill \& Newson $n$ Ward, A. W. $n$ Willey, A. G. $n$
Chattanooga, Hamilton co Chattanooga, Hamilton co Dover, Stewart co Columbia, Maury co Leiper's Fork, IVilliamson co Bartlett, Shelby co Jackson, Madison co Memphis, Shelby co Nashville, Davidson co Humboldt, Gibson co Franklin, Williamson co I 97 Main St., Memphis Nashville, Davidson co Loudon, Loudon co Manchester, Coffee co

\section{TEXAS.}

Black, Thomas $n$. Black, W. P. $n$ Bogarth \& Wimberly Boyd, W., \& Bro. $n$ Burr, H. B. $n$ Byers, Mrs. S. E. Dumas, J. W. $n$. Egerton, L. K. $n$. Johnson, J. R. $n$. Flake \& Co. $n$ Holmes, C. D. $n^{-}$. Kirkpatrick, G. W. n . Loessin, Herman $n$ Long, George A. n Madarasz, L. W. n McKee, G. A. $n$. Morrison, M. N. n Munson, T. V. $u$. Orne, E. J.

Radam, William $n$ Ramsey \& Son $n$. Sherman \& Collins $n$. Stell, W. W. $n$. . Stubenrauch, J. W. $n$. Vesey, F. W. $f$. Watson, William E. $n$. IVeymouth, J. E. n Whitaker, A. $f$

\author{
Ripley, Titus co \\ Double Springs P. O., Tarrant co \\ Wimberley, Hays co \\ Galveston, Galveston co \\ Dallas, Dallas co \\ Houston, Harris co \\ Thorp's Spring, Hood co \\ Denton, Denton co \\ Dallas, Dallas co \\ Galveston, Galveston co \\ Galveston, Galveston co \\ McKinney, Collin co \\ Black Jack Springs, Fayette co \\ Troup, Smith co \\ San Antonio, Bexar co \\ Larissa, Cherokee co \\ Corsicana, Navarro co \\ Denison City, Grayson co \\ Ysleta, El Paso co \\ Austin, Travis co \\ Mahomet, Burnet co \\ Sherman, Grayson co \\ Paris, Lamar co \\ Mexia, Limestone co \\ Waco, McLennan co \\ Brenham, Washington co \\ Corpus Christi, Nueces co \\ Houston, Harris co
}




\section{UTAH.}

Barker, J. M. $n$ Cliff, Edward $n$. De Shenning, G. "n Due, O. F. $n$ Johnston, G. W. $n$ Reading, John $f$. Ross, Andrew $n$. Smith, Henry $f$.
Willard, Box Elder co

- Mount Pleasant, San Pete co

- Salt Lake City

Salt Lake City

Huntington, Emery co

Salt Lake City

Washington, Washington co

Salt Lake City

\section{VERMONT.}

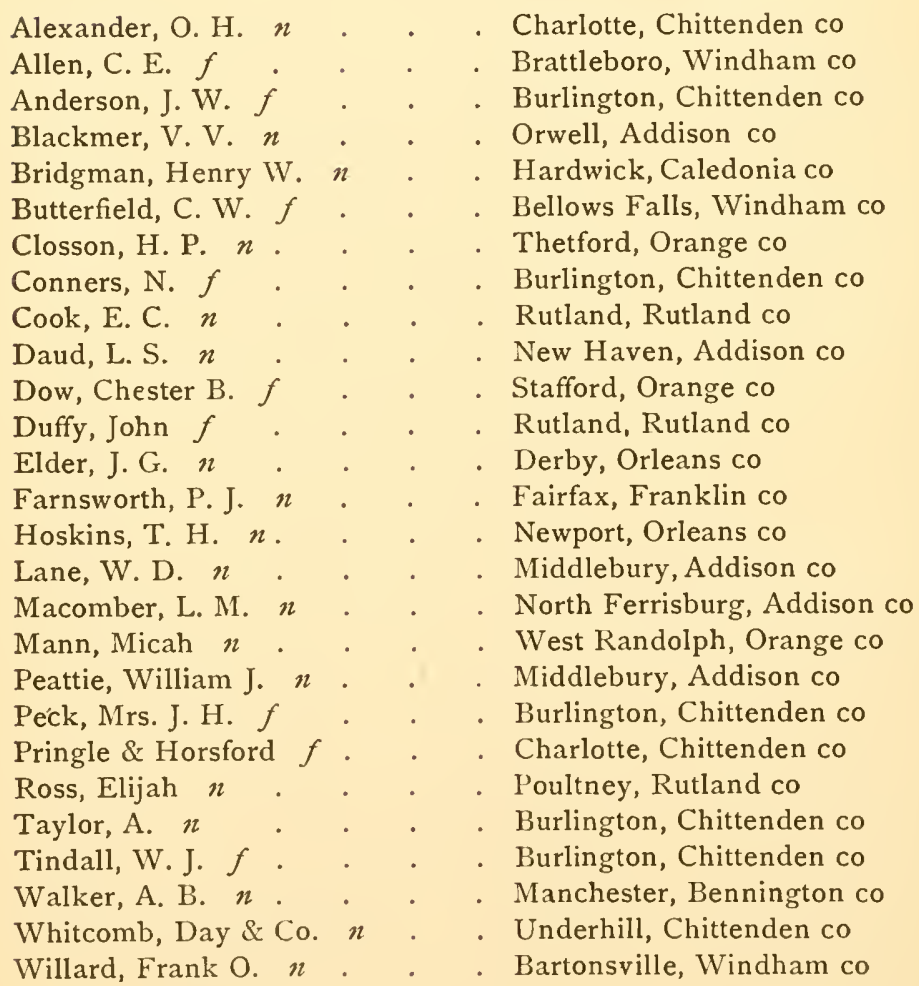




\section{VIRGINIA.}

Baker Floral Garden Bell, J. H. $n$.

Bonnot, J. N. $f$

Boswick, James R. $f$

Cantlin, H. A. $n$.

Davis, F., \& Co. $n$

Davis, Kent \& Hood $x$

Finch, Pleasant $n$

Fisk, Joseph $n$

Fry, James M. $f$

Grant, F. $f$

Halliday, John $n$.

Hammond, W. A. $f$

Harvey, J. J. $f$

Hill, J. L., \& Co. $n$

Hooper, John R. $f$

Kelly, W. B., \& Co. $n$

Laird, John $f$

Mallory, John A. $n$

Morton, John $f$

Mosby, J. D. $n$

Munson, D. O. $n$.

McGowan, James $n$

Pomeroy, John $f$.

Poytress, W. D. $f$.

Reynolds, M. $n$

Taggart, B. $f$

Tait, George, \& Son $u$.

Wheat, J. C. $n$

Wood, William R. $f$

Wright, P. S. $f$
Norfolk, Norfolk co

Harrisonburg, Rochingham co

Norfolk, Norfolk co

Portsmouth, Norfolk co

Richmond, Henrico co

Richmond, Henrico co

Richmond, Henrico co

Lynchburg, Campbell co

Guineys, Caroline co

Winchester, Frederick co

Forrest Depot, Bedford co

Lynchburg, Campbell co

Richmond, Henrico co

Richmond, Henrico co

Richmond, Henrico co

Richmond, Henrico co

Abington, Washington co

Richmond, Henrico co

Petersburg, Dinwiddie co

Richmond, Henrico co

Near Richmond, Henrico co

Falls Church, Fairfax co

Lynchburg, Campbell co

Alexandria, Alexandria co

Petersburg, Dinwiddie co

Norfolk, Norfolk co

Norfolk, Norfolk co

Norfolk, Norfolk co

Winchester, Frederick co

Norfolk, Norfolk co

Middletown, Frederick co

\section{WASHINGTON TERRITORY.}

Cook, A., \& Son $n$ Hudson, L. N. $n$. Jewett, A. H. . Ogle, J. M. $n$ Ritz, Philip n Starrett, George n
Vancouver, Clarke co

Tocoma, Pierce co White Salmon, Klikitat co

Puyallup, Pierce co

Walla Walla, Walla Walla co

Walla Walla, Walla Walla co 


\section{WEST VIRGINIA.}

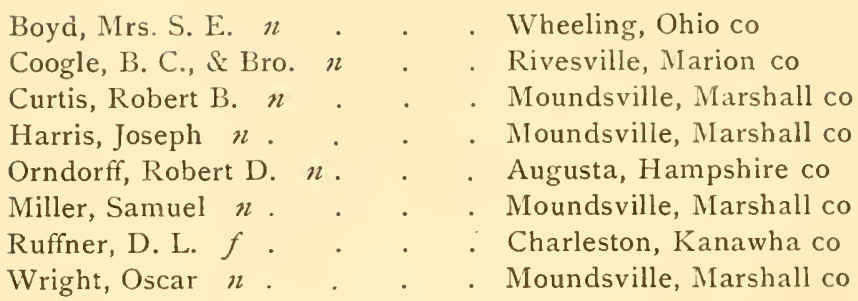

\section{WISCONSIN.}

Armstrong, David W. $f$ Armstrong, Thomas G. $f$ Ballantine, J. \& D. $n$. Baumbach, William $n$. Baumgarten, Charles $f$ Brainard, Anson $n$ Brainard, Edward M. n Bremer, J. $n$ Bronson, N. $n$ Chase, Mrs. J. M. Coe \& Converse $n$ Cowie Bros, $x$ Crocker, J. A. $n$. Currie Bros. $f$ Dalgleish, David L. $f$.

Daniels, E. IV. $n$.

Dilger, Frank P. $f$ Dorward, Martin B. $f$. Eliot, Edward $f$. Ellis, F. R. $f$

Enos, Horace, \& Co. $f$ Eskan, Frederick, \& Co. $f$.

Farnsworth, James $\mathrm{H}$. n "Fountaine Floral Co." $n$ Freeborn \& Hatch $n$. Freeman, S., \& Son $n$. Freytag, Jacob E. $f$.
- 144 Mason St., Milwaukee

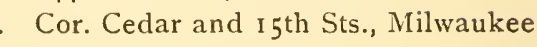

Bloomington, Grant co

- Wauwatosa, Milwaukee co

- East Division Market, Milwaukee

- Oshkosh, Winnebago co

- Oshkosh, Winnebago co

Stevens Point, Portage co

New Lisbon, Juneau co

Kenosha, Kenosha co

- Fort Atkinson, Jefferson co

Glencoe, Buffalo co

Kingston, Green Lake co Io8 Wisconsin St., Milwaukee Washington and Cold Spring Aves., Milwaukee

Aurorahville, Waushara co I26 Mason St., Milwaukee 1000 Humboldt Ave., Milwaukee 4I I Milwaukee St., Milwaukee Io86 Humboldt Ave., Milwaukee Milwaukee, Milwaukee co

Forrest Home Ave. and Burnham, Milwaukee

Fond du Lac, Fond du Lac co

Racine, Racine co

Ithaca, Richland co

Racine, Racine co

Forrest Home and 12 th, Milwaukee 
Grakow, Anthony F. $f$. Gray, J. W. n

Haentze, E. n

Haessler, Herman $f$.

Hoppenrath, Charles $f$

Hunt, Samuel $n$.

Huson, A. D. $n$

Johnson, C. A. $f$.

Jones, Isaac H. $f$.

Jordan, J. C. $f$.

Kellogg, George J. n

Kitzerow, William $f$

Knolle, T. $n$

Lang, F. N. n

Landreth, Albert $n$

Meidam, Dennis $f$

McColm, J. N. $n$.

Middlemas \& Laing $f$.

Miles, Isaac $f$

Palmer, N. N. $n$.

Paseo, J. L. $n$

Phoenix, F. K., \& Son n

Pinney, George $n$

Pinney, J. C. $n$.

Plumb, J. C., \& Son $n$.

Praefke, Frederick $f$.

Price, Thomas H. $f$.

Putnam, George W., \& Son n

Quabius, David $f$.

Rogers, W. H. $f$.

Sabin, R. B. $n$

Salzer, John A. $f$.

Sasse, Ferdinand $f$

Scherrmacher, C. W. $f$

Scherz, George $f$.

Sheibel, Frederick $f$

Smith, S. $f$

Springer, W. A. $n$

Stone, J. N. $n$

Thomann, Caspar \& Co. $f$.

Tuttle, A. G. $n$.

Van Loon, John $n$

IVagner, Joseph $f$

IVeeks, G. V. $n$

Wernich, Waldemar $f$.

Whitnall, Frank, \& Co. $f$

Wiesner, Frederick

Wilcox, E., \& Sons

Willan, Robert $n$.

Woodruff, Jacob, \& Son $f$
Appleton, Outagamie co

Trempealeau, Trempealeau co

Fond du Lac, Fond du Lac co

E. Division Market, Milwaukee

East Division Market, Milwaukee

Evansville, Rock co

Sheboygan Falls, Sheboygan co

Racine, Racine co

Milwankee, Milwaukee co

Watertown, Jefferson co

Janesville, Rock co

East Division Market, Milwaukee

Fillmore, Washington co

Baraboo, Sauk co

Manitowoc, Manitowoc co

Appleton, Outagamie co

Plymouth, Sheboygan co

392 Broadway, Milwaukee

Oshkosh, Winnebago co

Brodhead, Green co

Ripon, Fond du Lac co

Delavan, Walworth co

Sturgeon Bay, Door co

Sturgeon Bay, Door co

Milton, Rock co

East Division Market, Milwatikee

Lake Geneva, Walworth co

Ash Ridge, Richland co

East Division Market, Milwaukee

Appleton, Outagamie co

Sparta, Monroe co

La Crosse, La Crosse co

East Division Market, Milwaukee

East Division Market, Milwaukee

East Division Market, Milwaukee

Madison, Dane co

Racine, Racine co

Fremont, Waupaca co

Fort Atkinson, Jefferson co

East Division Market, Milwaukee

Baraboo, Sauk co

New Amsterdam, La Crosse co

Beaver Dam, Dodge co

Lyons, Walworth co

605 Grand Ave., Milwaukee

428 Milwaukee St., Milwaukee

East Division Market, Milwaukee

La Crosse, La Crosse co

New Lisbon, Juneau co

Ripon, Fond du Lac co 


\section{WYOMING TERRITORY.}

Chaffin, N. J. $f$. $\quad$. Cheyenne City, Laramie co

\section{SPECIAL NOTICE!}

The following publications are issued in connection with the "Horticultural Directory of the United States," and are especially commended to the trade:

\section{"SUPPLEMENT A,"}

which will be a list giving the names and post-office addresses of the Nurserymen, Florists, Fruitgrowers, and Seedsmen of England, Ireland, Scotland, Wales, and the Channel Islands, comprising in all about two thousand addresses.

The Supplement will be issued in pamphlet form, and will be sold to subscribers to the "Horticultural Directory" at one dollar (\$1.00) per copy; to non-subscribers at one dollar and fifty cents $(\$ 1.50)$ per copy, postage paid.

The trade must bear in mind that the rate of postage to points covered by the Supplement is only one cent for unsealed circulars weighing not more than two ounces. The business in Horticulture between the two English-speaking countries is yet in its infancy but is growing rapidly.

\section{"SUPPLEMENT B"}

will be issued April 15, 1885, and will comprise the names and addresses of the principal Nurserymen, Seedsmen, and Florists of Austria, Bavaria, Belgium, Denmark, France, Germany, Holland, Hungary, Italy, Poland, Russia, Saxony, Sweden and Norway, Switzerland, Wurtemburg, Brazil, India, Japan, Australia, New ;Zealand, and South Africa. Price sume as "Supplement A."

Subscriptions and remittances must be made to

$$
\begin{aligned}
& \text { ISAAC D. SAILER, Publisher, } \\
& \text { Union Insurance Co.'s Building, Third and Walnut Sts., }
\end{aligned}
$$

PHILADELPHIA, PA.

\section{PAPER SEFD BAGS.}

All kinds and sizes made to order for Seedsmen and Florists, blank or printed to order at the shortest motire and the lowest pricess.

\section{CLARK BROTHERS,}



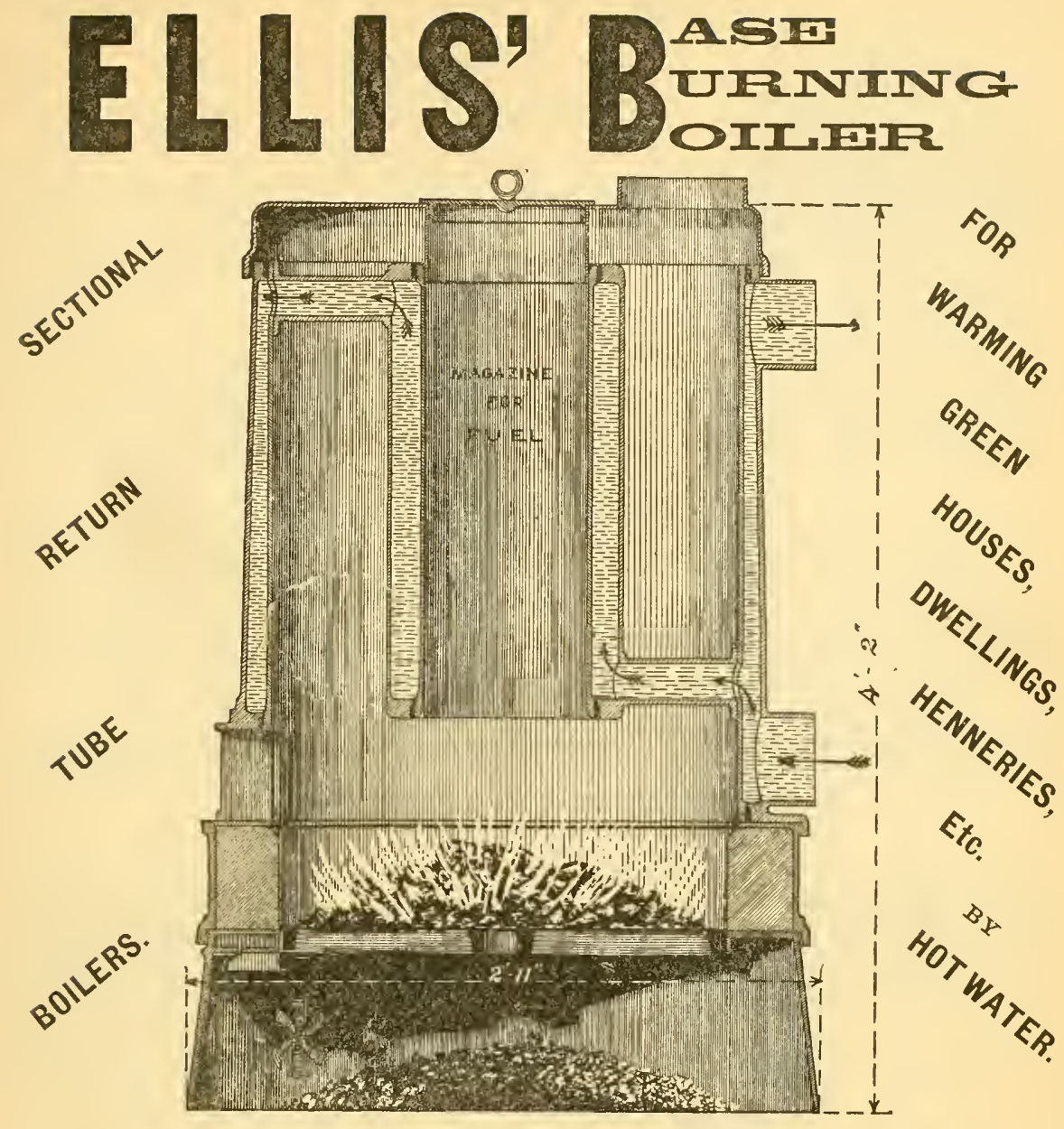

\section{STEAM BOILERS AND}

ENGINES.

\section{CHARIES R. FLIIS,}

182 Centre Street,

NEW YORK.

SEND FOR CIRCULAR. 


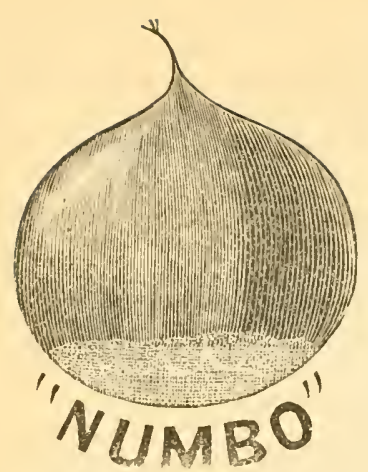

THE BIG CHESTNUT.

\section{SAMIUEL C. MOON,}

Morrisville, Bucks Co., Pa.

\section{MORRISVILLE NURSERY.}

"Num-bo" is an American seedling of the European Chestnut. The original tree is thirty years old, has never been injured by cold or disease, is a regnlar bearer, enormously productive, ripens early, and bears from

50 to 80 Quarts of Nuts Annually.

From 60 to 75 nuts make one quart. Grafted trees commence to bear in from four to six years. Send for descı iptive Catalogue.

ORNAMENTAL TREES A SPECIALTY.

WIIIIIA IM. IE. SIMIIIEI,

Late of the Firm of Henry A. Dreer,

$S$ IF ID DS IM A IV;

No. 1018 MARKET STREET, PHILADELPHIA.

Grower, Importer, and Dealer in Flower, Vegetable, and Field Seeds, Implemenis and Garden Requisites. Sepd Form and Trial Groutuds, Raduor, Pa.

Cutalogurs Free on Application.

\section{JAMMES P. FEIVITEI,}

Wire-Worker and Manufacturer of Flower Designs,

36 IV. Sixth Street,

PHILADELPHIA.

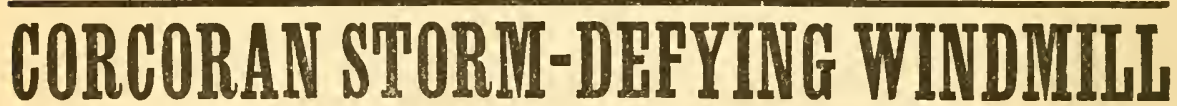

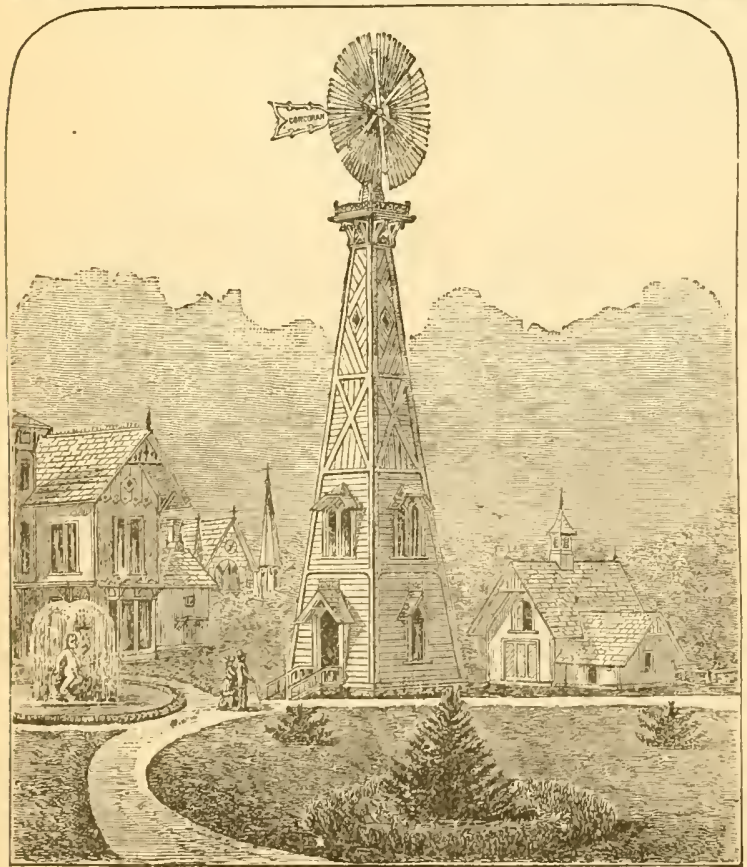

The following names comprise a few of the more prominent florists who use my system of water supply and to whom I refer. Send for catalogue explaining my method.

John Carton, Sea Bright, N. J.

Demaray \& Wheeler, Somerville, N. J. R. Free, Stamford, Conn.

Kretschmar Bros., Flatbush, L. I.

Siebrecht \& IVadley, New Rochelle, N. Y.

Charles Seiler, Savannah, Ga.

Homogansett Greenhouses, Wickford, R. I.

James Weir, Jr., Brooklyn, N. Y

John Boll, Jamaica, L I.

P. Caswell, Jr., Newport, R. I.

James Horan, Bridgeport, Conn.

Christolph Kunzel, Ridgewood, L. I.

G. Kıesling, Savannah, Ga.

G. Messeberg, Flatbush, L. I.

A. Roem, Union Hill, N. J.

James Weir \& Sons, Bay Ridge, L. I. Dietzenburger Bros, Bay Ridge, L. I. IV. C. Wilson, Astoria, L. I.

\section{A. J. CORCORAN,} 76 John St. New York City. 


\section{NEW FRUIT ANND ORNAMENTAL TREES.}

Send stamps for our Illustrated and Descriptive Catalogues. They contain full and accurate information about all the

\section{Old and New Fruits, Trees, Roses, Grape Vines, etc.}

with cultural directions, and are the most complete published. No. 1. Fruits, including small Fruits (new edition), Io cents. No. 2. Ornamental Trees, etc., I 5 cents. No. 3. Strawberries. No. 4. Wholesale. No. 5. Roses, a carefully compiled, systematized index, the only catalogue in which a variety can be found at a glance, free.

\section{ELLWANGER \& BARRY,}

Mt. Hope Nurseries.

ROCHESTER, N. Y.

\section{PARKER \& WOOD,}

\section{Agricultural Warehouse and Seed Store,}

Nursery Stock, Plants, etc.

Woodenware and Wood's Ice Tools,

No. 49 N. Market Street, BOSTON.

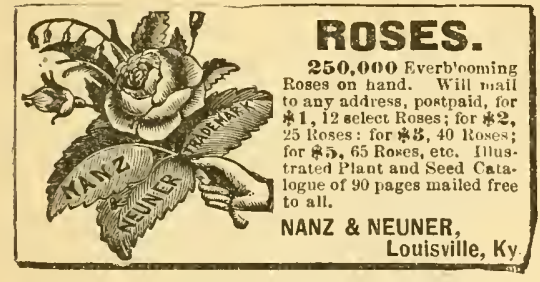

WHOLESALE AND RETAIL.

\section{Kissena Nurseries.} Trees and Plants.

Parsons\&Sons Co., LIMITE1).

Flushing, N.Y.
JOHN J. PERKINS,

Wholesale and Commission

\section{FLORIST,}

No. 839 SIXTH AVENUE,

(Bet. $47^{\text {th }}$ and $4^{8 \text { th }}$ Sts.)

NEW Y O R K.

BRANCH, 38 S. 16th St., Philadelphia, Pa.

Greenhouses, CRESKILL, N. J.

Consignments of Flowers Solicited. Write for Terms.

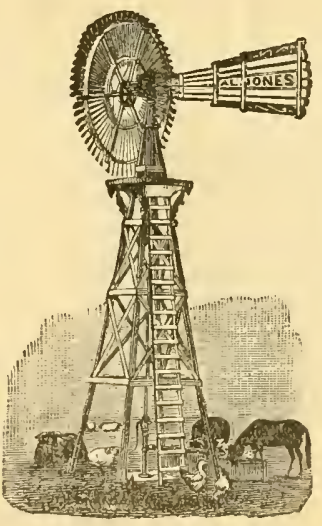

ARTESIAN

Well Borer

And Contractor for

WATER SUPPI,Y

FOR

Farms,

Dwellings,

Towns,

Villages, ANi)

PERKINS WIND MILL. Manufactories.

Philadelphia Agency of the

RUMSEY PUMP WORKS.

The largest in the world; over 800 different style Pumps; Hydraulic Rams, Wind Mills, Water Wheels, and a great variety of Pumping Machinery for Farmers' Dwellings, Country Residences, Stock Farms, Rallroads, etc. I deliver all goods free to the railroad or boats.

Plumbing and Steam-Heating In all its branches. Send for Catalogue, it costs nothing, to A. I. JONEs. Office and store,

114 NORTH SIXTH STREET,

Warehouse and Factory, 628 Race St, Philada. 


\section{Green House Heating Apparatus. SCOLLAY'S}

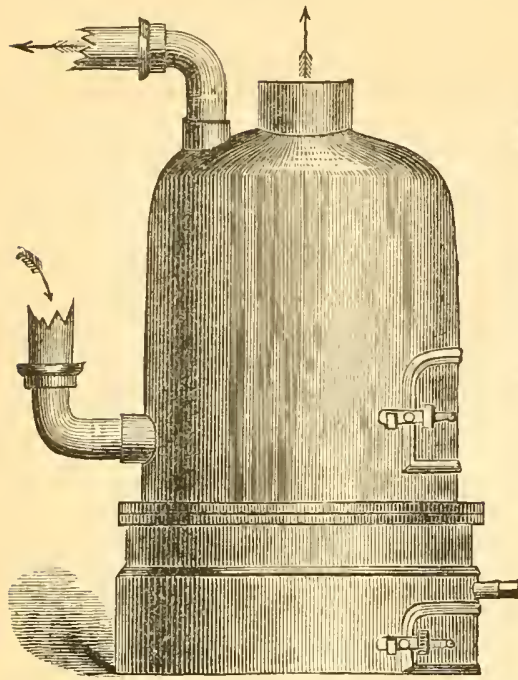

Patented Improved Portable and Sectional Boilers.

Heats from $150 \mathrm{ft}$. to $5000 \mathrm{ft}$, 4 -inch pipes.

\section{Patent Rubber Plant Sprinkler}

OVER 40,000 NOW IN USE.

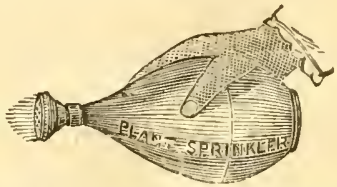

Diploma awarded by N. Y. Horticultural Society. Illustrated and Descriptive Catalogue Free on Application.

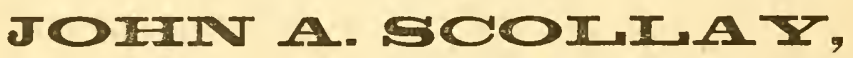

74 and 76 Myrtle Ave., Brooklyn. N. Y.

PAIR HIL, TERRA-COTTA WORKS. JACOB C. CASSEL, 2341 N. 7th St., Phila., Pa.
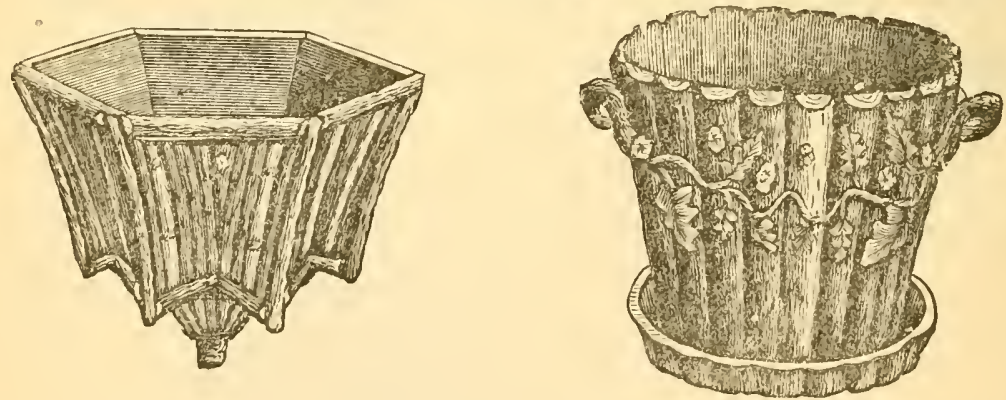

MANUFACTURER OF

FLORICULTURAL TERRA-COTTA WARE, GARDEN VASES, FERNERIES, FLOWER POTS, HANGING BASKETS, RUSTIC AQUARIUMS, AQUARIUM ORNAMENTS and other NOVELTIES, AND DEALER IN

GLASS SHADES, FISH GLOBES, FLOWER-POT BRACKETS, Etc.

STORE, 1207 Columbia Avenue.

These goods are more Pructical, Artistic, and Durable than any others munfactured for the same purpose. Illustruted Catulogue "pon Application. 


\section{ORNAMENTAL}

\section{FOLIAGE PLANTS,}

Greenhouse Plants,

\section{Bedding Plants, Etc.}

CATALOGUE MAILED ON APPLICATION.

\section{DAVID FERGUSSON \& SONS,}

Ridge and Lehigh Aves.

PHILADELPHIA, PA.

\section{CRAIG \& BROTHER,}

49th and Market Streets, West Philadelphia. - R O S ES.

All the leading forcing kinds in exceptionally fine condition, including "MARVEILLE DE LYONS."

The extraordinary New Hybrid Rose, "HER MAFESTY,"

Will be offered this Fall. Write for circular and particulars.

"WM. FRANCIS BENNETT,"

The famous Crimson Hybrid Tea.

"NEW CARNATION SCARLET GEM."

The best and most prolific Scarlet Carnation. Young plants in Spring, \$10.00 per hundred; out of ground in Fall, \$15.00 per hundred.

ASPARAGUS TENUISSIMUS.

The largest stock in this country. Prices low.

$$
\text { FERNS. }
$$




\section{Those Who Buy}

\section{PLANTS AND NURSERY STOCK}

ARE the GARDENERS,

ARE the FLORISTS,

ARE the AMATEURS,
ARE the FRUITGROWERS,

ARE the GENTLEMEN FARMERS

ARE the MARKET GARDENERS,

\section{WHO READ}

\section{"The

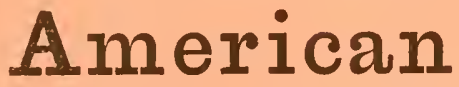 \\ Garden,"}

ESTABLISHED 1872. EDITED BY DR. F. M. HEXAMER.

A MONTHLY ILLJSTRATED MAGAZINE OF HORTICULTURE.

IT IS NOT

Connected with any mercamile hous.

An organ of any merchant.

An advertising sheet.

IT IS

An independent Jogrnal.

Devoted wholly to horticulture.

The organ of fruit, flower, and vegetable growers.

Edited and written by horticulturists.

IT ASKS

The cordial coöperation of horticulturists in sub-
scriptions and advertisements. A good word for it to your friends.

\section{IT HAS}

No a xes to grind.

Nothing to sell.

Nothing to puff.

IT WILL NOT

Puff unworthy varieties.

Admit fraudulent advertisements.

IT WILL

Give every promising new variety and method a fair hearing

Expose fraud of every degree.

Stand fearlessly for truth.

Promote all good work in horticulture.

Be improved from month to month.

Deserve support

ITS CONTRIBUTORS ARE SUCH MEN AS PETER HENDERSON, JOSIAH HOOPES, WM. FALCONER,

S. PARSONS, Jr.. E. P. ROE, M. MILTON, W. C. BARRY, MARSHALL P. WILDER, E. S.

RAND, E. D. STURTEVANT, CHAS. H. SHINN, A. S. FULLER, T, H. HOSKINS,

"ELM," and a host of the most practical Horticulturists in America.

It is a real treasure. * * It stands in the front rank and merits success.-Marshall $P$. Wiuder.

Its teachings are sound and practical $-A \mathrm{~m}$. Agriculturist.

Eminently practical, trustworthy, and enjoyable.-Nei' York Tribune.
Very useful.-Gardener's Monthly.

Without exception the best paper devoted to general horticulture.-C. L. Allen, Long Island.

Contains just what the people want, served in the most palatable style.-P. T. Quinn, Nero jersey.

Subscription Price only \$1.00 a Year, which is very low for this elegantly gotten up magazine. It is certainly the best printed and handsomest journal of either horticulture or agriculture in America.

Specimen Copy 10 Cents, which may be deducted from the subscription when sent. Address,

\section{E. II. IIIE IZ,}

New York or Greenfield, Mass.

We offer $\$ 600.00$ in prizes to the introducers of the best varieties of Grape, Strawberry, Raspberry, Blackberry, and Gooseberry. 
This blustration show's the cover-page, and is one-fourth its actual size.

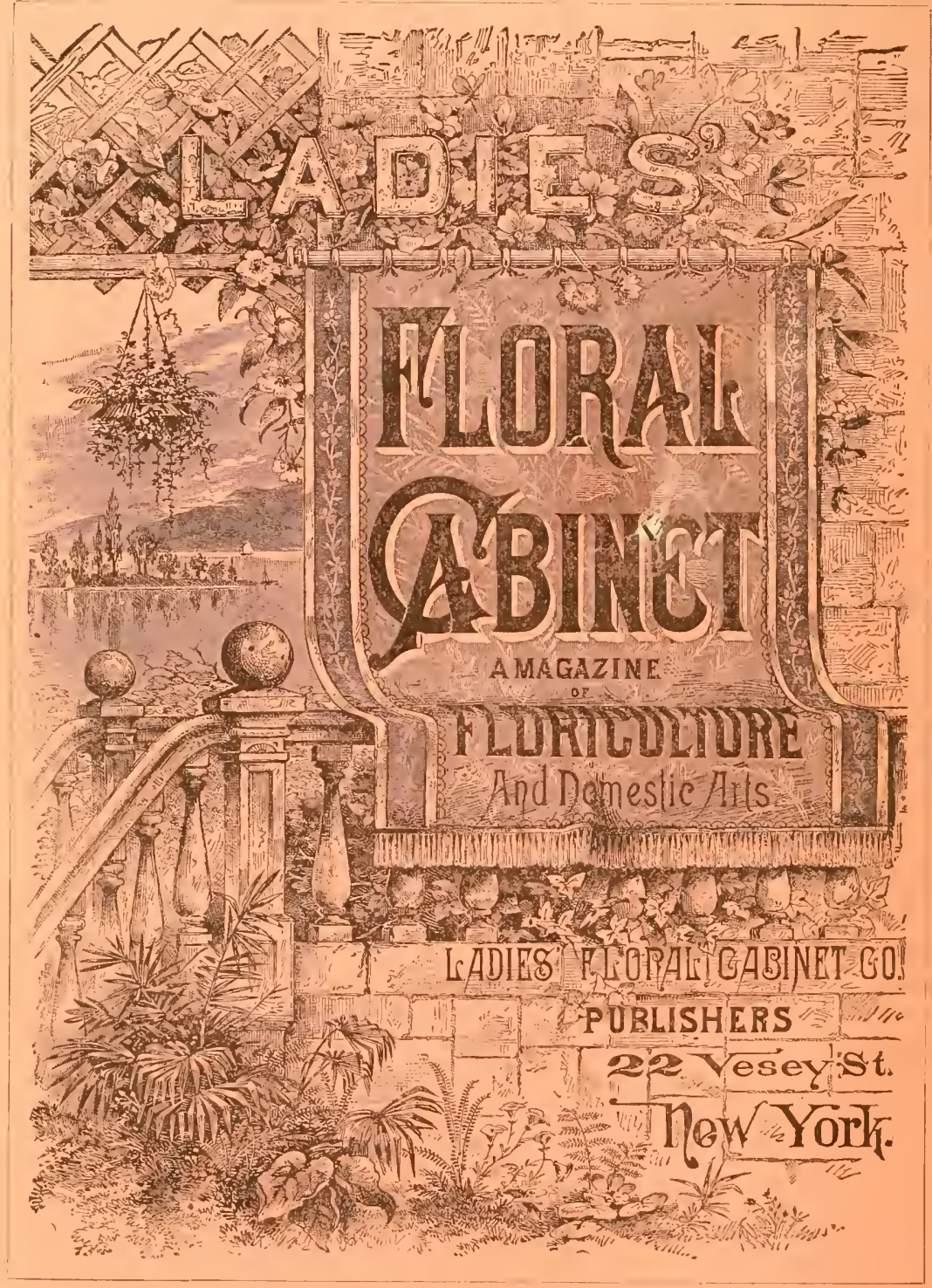

MONTHLY-\$1.25 PER YEAR.

The only American Magazine devoted to FLORICULTURE for AMATEURS which is entirely disassociated with any Florist's, Seedsman's or Nurseryman's interests. 


\title{
TH E
}

\section{Gardeners' Monthly}

AND

\section{HORTICULTURIST.}

\section{EDITED BY THOMAS MEEHAN,}

\author{
STATE BOTANIST OF PENNSYLIANIA.
}

\section{GENE AL MONTHLY CONTENTS.}

FLOWER GARDEN AND PLEASURE GROLND.

GREENHOUSE AND HOLSE GARDENING

FRUIT AND VEGETABLE GARDENING.

NEW AND RARE PLANTS AND FLOWERS

NEW AND RARE FRUITS AND VEGETABLES

PERSONAL NOTES OF THE EDITOR, AND BEST ORIGINAL WRITERS. FORESTRY, BOTANY, HORTICULTURAL LITERATURE.

SELECTIONS FROM COVTEMPORARY AUTHORITIES. HORTICULTURAL SOCIETIES.

HINTS FOR THE MONTH AND SEASON, FOR THE AILATEUR, FLORIST, FRUIT GROWER, ETC.

This Magazine is indispensable to all who are interested in Horticulture in the least degree; whether the Novice caring for the proper welfare of a single plant; the enthusiastic Amateur with greater or less means to indulge a growing interest; or the Scientist, already abreast with the present stores of Horticultural knowledge. All will alike find the able and intelligent correspondence and discussion in our monthly issues a source of unfailing and ever new interest. The modest query and the reported observations of experience receive equal welcome, respect, and attention.

Sample copies will be mailed for 18 cents in postage stamps.

\section{Subscription Price, $\$ 2.00$ per Year.}

Trwo New Subscribers, or One Renewal and One New for $\$ 3.00$. Five Subscribers for $\$ 7.00$.

Same proportionate rates for fractions of a year. On Foreign subscriptions add to these prices 48 cents each. per yearly subscriber, for foreign postage.

Remit by Post-office order or Registered Letter, or Draft on New York, Philadelphia, Middle States, New England or Baltimore. Address,

Chas. H. Marot, 8 i 4 Chestnut St., Phila. 


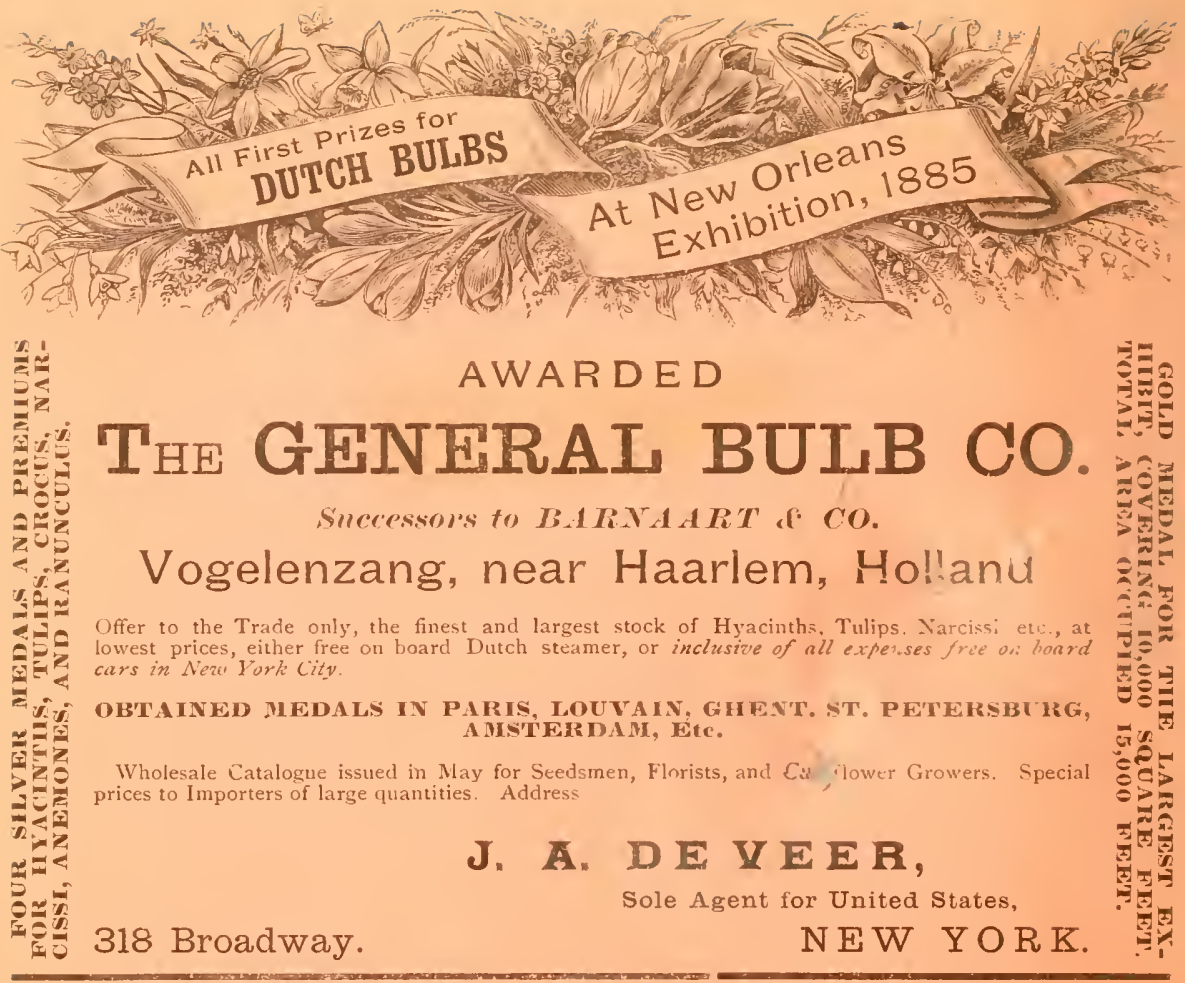

RICHARD SMITH \& CO., Worcester, ENGLAND.

Clematis Jackmanii, $\$ 15.00$ per 100; best varieties, seller's choice, $\$ 1650$; ditto purchaser's, $\$ 18.75$ per zoo. Roses (20 acres), hybrid perpetuals, very strong, $\$ 7.50$, seller's selection; \$10.00, purchaser's.

Roses, tea-scented, etc., in pots, very fine, $\$ 15.00$ and $\$ 18.75$. New and Scarce Roses, $\$ 450$ per dozen. Gooseberries, $\$ 400, \$ 500$, and $\$ 6.25$ per 100 ; show and rare varieties, $\$ 7.50$.

Ampelopsis Veitchii, $\$ 10.00$ and $\$ 500$ per 100. (These are from cuttings, and therefore true.) Birch, fern-leaved weeping, 6 to 12 feet, from $\$ 4.00$ to $\$ 6$ oo per dozen.

91 Acres of CONIFERS, ORNAMENTAL TREES, and FLOWERING PLANTS. $31 / 2$ Acres of GLASS for STOVE and GREENHOUSE STOCK.

HERBACEOUS, ALPINE, and OLD ENGLISH PLANTS OF MERIT in great variety FRUIT TREES, VINES, RASPBERRIES, STRAWBERRIES, Etc. of the choicest kinds.

IDESCIIIPTI IE LISTS, containing a vast amount of information, FREE.

\section{CHARLES E. PENNOCK, Wholesale Florist, 30 South Sixteenth Street,} PHILADELPHIA, PA.

Consignments solicited.

Flowers shipped to all points. 


\section{The Union Insurance Co.}

OF PHILADELPHIA.
No. 3c
street.

\section{FIREMMARINE, AND INLAND INSURANCE?}

Risks Taken on Gireenhouses when Heated by Siezril or Hot Water.

\section{Assets, January I, i $885, \$ 866,429.80$.}

Government, City of Philadelphia, and other Bonds, market value,

Bank and other Stocks, market value,

Bills Receivable for Marine Premiums and Book Accounts due Company,

First Mortgages on City Property,

Real Estate owned by the Company,

Cash in Bank and Office, and Loans with Collaterals,

$$
\begin{aligned}
& \$ \div 89,25630 \\
& \text { I } 20,75900 \\
& \text { I } 54,06 \text { I } 6 \text { I } \\
& 40,00000 \\
& \text { I 72, I } 9066 \\
& \frac{90,16223}{\$ 866,42980}
\end{aligned}
$$

Losses Paid since Organization, \$13,010,805.12.

Dividends Paid since Organization, \$1,860,331.00.

\section{Liabilities.}

Reserve for Reins:-

Reserve for Losis

Unclaimed Dividends, .

Surplus as to Policy Holders,

$$
\begin{array}{rr}
\$ 410,130 & \text { I } 5 \\
74,148 & 38 \\
2.480 & 33 \\
379,670 & 94 \\
\hline \$ 866,429 & 80
\end{array}
$$

W. S. HA.SAAT. . President.

Cimarles S. Holduximean,

Jonx M. Cowel,

Manager Fire Department. 


\section{TOUTEXIR'S FOOD FOR FLOWLRS \\ -...AMMONIATED

A Fentilizer made especially for Plants grown in the H. ns, Garden, or Conservatory; clean, free from offenstve orlor, largely soluly il iviter, and pro-

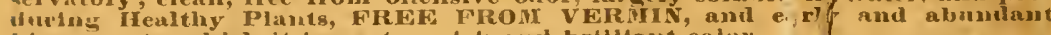

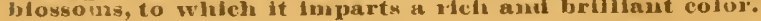

PIRICE LTST.-No. I. TRIAL PACKAGE, sufficient for twenty Pla s F Three Monting, Sent by mail postage prepaid, on receipt of price. No. 2. REt PACKAcis, Suffici for twenty Plants ior Ome Year, 35 cents. Sent by mail, postage prepaid, a recy t of price

PACKACRS 13\% MAIL.-This Dressing is sold by Florists, Druggista, fni Seedsmen gener atly ; but if it is not obtainable in the place where you live, to not inccest un imita.ib $z$, but send directly to us for a package by nail. The packages sent by mail are made of tin, and fonvey the contents afely. 'Two ten-cent piecus for the No, I size, or a twenty-five and a ten-cent pi-ce for ti.e No. 2 size, wrapped in thin paper, and enclcan in a luttur, will reach us safely.

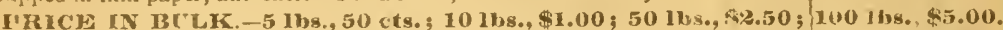

When sold in bulk, it is packed in strong bags, and ay be sent by express or freir't to any part of the country. Any quantity under ju lbs. should be sent by express. We mak no i.ıa.ge for packages $\because$ fur delivery to express or depot. Adulress

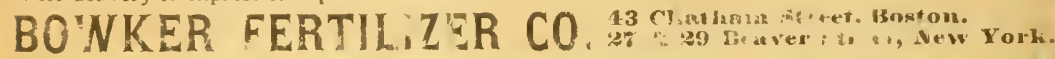

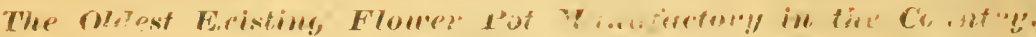
ESIAEIISETE: 1765.

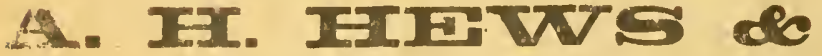

MANUFACTURERS OF HAND AND MACHINF: MADE

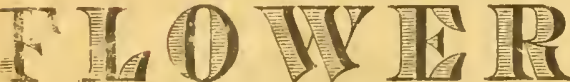

Orelilis Pots in Stock and to Order.
और

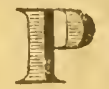

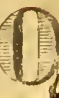

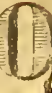

Art Pottery for Amatera, wintiug.

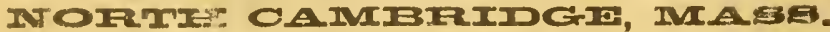

E!l ordars promptly atteuded to.

We pack with care for shipping.

ILLUSTIATED CATALOGUES ON APPLICATION.

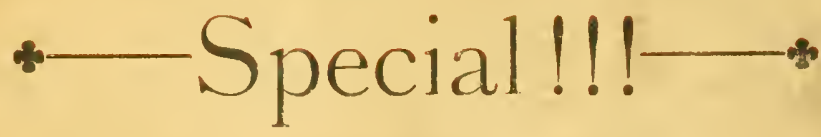

Subscribers to and Advertisers in this "Horticultural Directory of the United States" are respectfully requested to aid the publisher in perfecting this book.

A correct Direciory means the saving of thousands of dollars a year now uselessly spent in postagre.

The first Correcticn Sincet (price, \$1.00 to subscribers tr, the "Directory") will be $\therefore . ., 1$ in the latter part of this

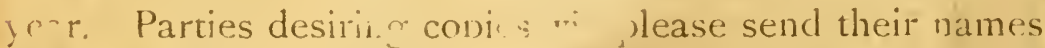
for : "cristration, as only a sificient number to supply suhseribers will be finted.

IS:AC I SAII, Pr. Publisher,

$$
\text { ronun Incmal . Tindung, Thud and Walnut Sts. }
$$

